\title{
ASAS Light Curves of Intermediate Mass Eclipsing Binary Stars and the Parameters of HI Mon
}

\author{
S. J. Williams, ${ }^{1}$ D. R. Gies \\ Center for High Angular Resolution Astronomy and \\ Department of Physics and Astronomy, \\ Georgia State University, P. O. Box 4106, Atlanta, GA 30302-4106; \\ swilliams@chara.gsu.edu,gies@chara.gsu.edu \\ J. W. Helsel \\ Department of Physics, Furman University, \\ 3300 Poinsett Hwy. Greenville, SC 29613; \\ john.helsel@furman.edu \\ R. A. Matson, S. Caballero-Nieves ${ }^{1}$ \\ Center for High Angular Resolution Astronomy and \\ Department of Physics and Astronomy, \\ Georgia State University, P. O. Box 4106, Atlanta, GA 30302-4106; \\ rmatson@chara.gsu.edu,scaballero@chara.gsu.edu
}

\begin{abstract}
We present a catalog of 56 candidate intermediate mass eclipsing binary systems extracted from the 3rd data release of the All Sky Automated Survey. We gather pertinent observational data and derive orbital properties, including ephemerides, for these systems as a prelude to anticipated spectroscopic observations. We find that 37 of the 56 , or $\sim 66 \%$ of the systems are not identified in the Simbad Astronomical Database as known binaries. As a specific example, we show spectroscopic data obtained for the system HI Mon (B0 V + B0.5 V) observed at key orbital phases based on the computed ephemeris, and we present a combined spectroscopic and photometric solution for the system and give stellar parameters for each component.
\end{abstract}

Subject headings: stars: binaries - eclipsing, stars: individual (HI Mon)

\footnotetext{
${ }^{1}$ Visiting astronomer, Cerro Tololo Inter-American Observatory, National Optical Astronomy Observatory, which is operated by the Association of Universities for Research in Astronomy, under contract with the National Science Foundation.
} 


\section{Introduction}

Eclipsing binaries are a major source of fundamental astrophysical parameters of stars such as mass and radius. These parameters can help us better understand and constrain the overall picture of stellar evolution and its consequences, including but not limited to exoplanet host star parameters and studies of Galactic and extragalactic star formation and abundance evolution. In a recent review, Torres et al. (2010) list 190 stars with mass and radius known to better than $\pm 3 \%$, only 26 ( or $\sim 14 \%$ ) of which have a mass greater than 3 $M_{\odot}$. This paucity of data for intermediate to high mass stars in binary systems is alarming. In particular, binary star evolution of systems of this type are one formation channel for type

Ia supernovae (Meng \& Yang 2010), one of the most studied phenomenon in determining cosmological parameters. In recent years large scale photometric surveys such as the All Sky Automated Survey (ASAS; Pojmański 2002) have preceded other surveys looking for transiting exoplanets. The byproduct of these searches will be a very large number of well sampled eclipsing binary light curves. The impetus behind this work is to find candidate intermediate and high mass binary systems with the future goal of obtaining fundamental parameters by observing these systems spectroscopically and resolving double lines. The applicability and validity of this technique has been demonstrated for lower mass systems in the recent effort by Deb \& Singh (2011).

We are starting a systematic observing program of newly identified or neglected intermediate mass eclipsing binaries. As an example of the kind of analyses we wish to pursue, we show here a case study of the binary system HI Mon (HD 51076). This system's variability was first noted in Wachmann (1968). The system was classified as an "OB" type system in Vogt (1976) and has been listed as an early B-type system since Eggen (1978), although no spectra for the system have been published. Spectroscopic investigations of such binaries with hot components are a critical part of any parametric analysis since the photometric colors are similar for all hot stars and are influenced by interstellar reddening.

The extraction and selection of candidate massive eclipsing binary light curves is described in Section 2. This is followed in Section 3 by a discussion of the analyses of these systems and estimation of uncertainties associated with computed values. Section 4 discusses the methods and results of the combined light curve and spectroscopic analysis performed on HI Mon. 


\section{Sample Selection}

The sample selection criteria are based upon an ongoing spectroscopic observing program with the DeVeny Spectrograph (formerly the White Spectrograph on the $2.1 \mathrm{~m}$ telescope at Kitt Peak National Observatory) on the $1.8 \mathrm{~m}$ Perkins telescope at the Anderson Mesa Station of Lowell Observatory. We searched the All Sky Automated Survey Catalog of Variable Stars (Pojmański 2002, 2003; Pojmański \& Macieiewski 2004, 2005; Pojmański et al. 2005) which is a subset of the 3rd data release (Pojmański 2002).2 The ASAS Catalog of Variable Stars lists preliminary orbital periods for the identified eclipsing binaries. Furthermore, Pilecki, Pojmański, \& Szczygieł have prepared an on-line catalog 3 that provides estimates of the system parameters based upon the $V$ and $I$-band light curves. We visually inspected several thousand individual light curves and extracted photometry for those systems that met our specific criteria. First, systems were selected that were typically north of $-25^{\circ}$ with a few exceptions near this cutoff limit. The most southern object is HD 122026 at $-27^{\circ}$ while the most northern object is DR Vul with a declination of $+26^{\circ}$. The data releases so far from the ASAS have reached a northern limit of $+28^{\circ}$, a hard limit for the time being. In order to meet the exposure time limits for our spectral resolving power of $R=\lambda / \Delta \lambda \gtrsim 5000$, we adopted a limiting $V$ magnitude of 11 . We then selected only light curves that had a primary eclipse depth greater than 0.1 mag to include only systems with well defined photometric variations. In addition, to ensure that the secondary spectral lines will be readily detected, we chose light curves where the ratio of eclipse depths was not extreme. We avoided contact systems and selected those with "EA" and "EB" light curves in the definition of Giuricin et al. (1983) because we want to study systems where the stars have yet to interact with each other and have evolved thus far as individual stars.

With this first cut of light curves, we then found the corresponding $J, H, K_{s}$ magnitudes from the 2MASS catalog (Skrutskie et al. 2006) for each object. Using the maximum brightness from the ASAS $V$-band light curve and the 2MASS $K$ magnitude, we computed $V-K$ colors for each candidate object. For many objects there is an unknown amount of interstellar reddening, which will cause the $V-K$ color to increase and indicate a star with a cooler spectral type. Therefore, to look for intermediate mass binary candidates $\left(\gtrsim 3 M_{\odot}\right)$, we used Table A5 in Kenyon \& Hartmann (1995) to eliminate later spectral types based on the apparent $V-K<0.7$, corresponding to a spectral type earlier than F0 V. To add an additional constraint, we also computed the reddening-free $Q$ parameter in the infrared from Comerón et al. (2002) with the 2MASS values. Stars with $Q<0.1$ usually correspond to

\footnotetext{
${ }^{2}$ http://www.astrouw.edu.pl/asas/?page =main

${ }^{3}$ http:/www.astrouw.edu.pl/asas/?page=eclipsing
} 
main sequence stars with mass greater than $1.4 M_{\odot}$.

Observational parameters for candidate systems meeting all criteria are listed in Table 1. Column 1 lists the ASAS identification tag in the ASAS catalog of variable stars. In column 2 we list an alternate object name, typically found in the Simbad database. Following this, columns 3 and 4 contain the right ascension and declination of the objects in the 2000.0 epoch as found from the Simbad Astronomical Database. Columns 5 and 6 list the $V-K$ and $Q$ values as discussed above. Next is column 7, showing the spectral classification found in the literature for each object while column 8 lists the reference for each entry. Column 7 demonstrates that most of the systems are intermediate mass binaries with an A- or B-type primary star. Column 9 lists whether the system was known to be a binary before the ASAS Catalog of Variable Stars and gives the reference to the work where the system was first discovered to be a binary. We wish to note that only 21 of our 56 candidates listed in Table 1 are contained in the on-line atlas of Pilecki, Pojmański, \& Szczygieł.

\section{Light Curve Analysis}

For each system, we fit the $V$-band light curve using the Eclipsing Light Curve (ELC)

code of Orosz \& Hauschildt (2000). The program solves for orbital and physical parameters relevant to the light curve. These parameters include the epoch of mid-eclipse of the primary star $T_{\mathrm{o}}$, the orbital period $P$, the orbital inclination $i$, the Roche lobe filling factor of each star, the temperature of each star, and the eccentricity and longitude of periastron of the primary $\omega$ in cases with elliptical orbits. In ELC, the primary is defined as the more massive star. It should be noted that the sizes and temperatures of the stars are relative, and without spectroscopic analysis, we cannot determine their true values.

In finding a best fit light curve for each system, our solutions with ELC create $\gtrsim 40,000$ simulated light curves and record the $\chi^{2}$ statistic associated with each light curve. We project $\chi^{2}$ over the range of each parameter of interest and focus on the region around $\chi_{\min }^{2}$. The resulting $\chi^{2}$ curves are not symmetric about the minimum, so our final estimate for the uncertainty of any particular parameter was the larger of the two adjoining $\chi_{\min }^{2}+1$ values. The best fit values are listed in Table 2, with uncertainties listed in parentheses in units of the last digit quoted. Column 1 in Table 2 lists the ASAS ID and column 2 gives the alternate ID. The orbital period for each system is given in column 3. The period uncertainties are smaller in the shorter period systems due to the large number of orbits covered in the ASAS. Similarly, column 4 is the epoch of mid-eclipse of the primary. Note that this is different than the epoch of periastron for binaries with eccentric orbits. The ASAS Catalog of Variable Stars also lists $P$ and $T_{\mathrm{o}}$. Our orbital periods do not differ greatly, but our reference epochs 
were chosen to be more recent than those listed in Pojmański (2002), reflecting times closer to when spectroscopic observations may be taken. Next is the orbital inclination in column 5 , followed by the eccentricity of the orbit in column 6, and the longitude of periastron in column 7, where applicable. The value of the eccentricity found for BD+11 3569 of $0.006 \pm 0.002$ is only $3 \sigma$ different than zero. We therefore estimate that given the typical uncertainty in the ASAS data, any system listed with $e=0.0$ probably has an eccentricity no greater than 0.01. It should also be noted that the longitude of periastron given here follows the spectroscopic orbit definition, i.e., measured from the plane of the sky through the center of mass to the primary.

To check the accuracy of our results, we can compare our values listed in Table 2 with those previously published. For example, a detailed light curve study of YY Sgr was made by Lacy (1993). His fit gives a longitude of periastron of $214.52 \pm 0.46 \mathrm{deg}$, matching with our value of $214.1 \pm 0.5 \mathrm{deg}$ within uncertainties. The eccentricity in Lacy's work of $0.1573 \pm$ 0.0008 and the inclination of $88.89 \pm 0.12 \mathrm{deg}$ are close to our derived values of $0.117 \pm 0.001$ and $88.1 \pm 0.1 \mathrm{deg}$, respectively, but still differ by more than $3 \sigma$. This is most likely due to the differences in the data analyzed. The data set in Lacy (1993) consists of 717 measures most of which were made during eclipses where the above parameters are best constrained. Our data set includes 601 measures more or less evenly distributed around the orbit, and thus eclipses are not as well measured and our resulting parameters are not as accurate nor as well constrained. Future observations, specifically targeted to get data during eclipses, will help resolve these differences.

One of our goals in selecting the candidates in Tables 1 and 2 is to collect a set of data for early-type eclipsing binaries. Using previously published spectral classifications we construct a histogram of the number of objects as a function of spectral type in Figure 1. This plot may be somewhat biased, as several spectral types are from Cannon \& Pickering (1924). Subtle differences in the He I $4471 \AA$ line are the determinant for discerning late-B from early-A spectral types. Early photographic plate spectra may not have been sufficiently sensitive to the changes in this temperature region, and we suspect several systems are consequently lumped into the "A0" category. Regardless, this plot shows that for the objects with published spectra, we have met our goal of finding systems earlier than F0. In fact, we have more stars (30) hotter than A0 than stars (17) cooler than A0. Note that HD 60389, with a spectral type estimate of G6 V does not appear in this figure. Note also that not all systems discussed here have spectral types, and some previously known systems have spectral type estimates for both stars plotted in the histogram. Light curve solutions for the full sample are given in the Appendix. 


\section{HI Mon Spectra}

Spectra for HI Mon were obtained at the Cerro Tololo Inter-American Observatory (CTIO) 4-m Blanco Telescope between 2009 Dec 23 and 2009 Dec 27. The RC-spectrograph was used with the G380 (1200 grooves $\mathrm{mm}^{-1}$ ) grating in second order and blue collimator giving wavelength coverage from 3932 to $4750 \AA$ with a three pixel resolution of $R=\lambda / \delta \lambda=$ 5400. Exposure times ranged from 150 to $180 \mathrm{~s}$ in order to obtain a signal-to-noise ratio $(\mathrm{S} / \mathrm{N})>100 \mathrm{pixel}^{-1}$. Helium-Neon-Argon comparison lamp spectra were obtained before and after each target observation, and bias and flat-field spectra were obtained nightly. The spectra were extracted and calibrated using standard routines in IRAF4. Each continuumrectified spectrum was then transformed to a common heliocentric wavelength grid in $\log \lambda$ increments.

To compute important astrophysical parameters for individual stars in double-lined spectroscopic binaries that are also eclipsing, spectra need to be obtained near quadrature points (times of maximum velocity separation). This will allow for the estimation of velocity semiamplitudes for each star in the system, and leads directly to an estimate of the mass ratio. To observe the binary system in such a configuration, one needs to observe the system at very specific times in its orbit. Therefore, an accurate ephemeris from the more easily obtained photometric light curve data is desirable. Using this ephemeris for HI Mon as computed above, we observed the system near quadrature phases in order to test both the accuracy of our ephemerides and to test whether this approach can yield accurate $(<3 \%$ uncertain) astrophysical parameters for stars. Two such observations near quadrature points (orbital phase 0.31 and 0.75 ) are shown in Figure 2 along with identified spectral features.

\subsection{Radial Velocities}

Radial velocities were measured using four spectral lines, He I $\lambda 4026, \mathrm{H} \delta \lambda 4101$, He I $\lambda 4143$, and $\mathrm{H} \gamma \lambda$ 4340. A template-fitting scheme (Gies et al. 2002) was used that measures velocities by using model spectra weighted by a flux ratio to match both the shifts and line depths in the observed spectra. We found no evidence for emission or intrinsic line asymmetries in any spectral feature.

The BSTAR2006 grid of stellar models from Lanz \& Hubeny (2007) was used to derive

\footnotetext{
${ }^{4}$ IRAF is distributed by the National Optical Astronomy Observatory, which is operated by the Association of Universities for Research in Astronomy (AURA) under cooperative agreement with the National Science Foundation.
} 
template spectra. These models are based on the line-blanketed, non local thermodynamic equilibrium (NLTE), plane-parallel, hydrostatic atmosphere code TLUSTY and the radia-

tive transfer code SYNSPEC (Hubeny 1988; Hubeny \& Lanz 1995; Hubeny et al. 1998). The process of finding templates began by making initial estimates for temperatures, gravities, projected rotational velocities, and relative flux contributions from each star. Parameters for model templates were then checked against spectral features in the tomographically reconstructed spectra of each star (Section 4.2). The parameters were changed and new templates made after initial fits to the light and radial velocity curves (Section 4.3) indicated that slightly different values were more appropriate. The velocity analysis was performed again until the best fit was obtained. Based on the relative line depths of spectral components, we were able to obtain an observational monochromatic flux ratio in the blue of $F_{2} / F_{1}=0.70 \pm 0.05$.

The four values for velocity from each spectrum for each component were averaged, and the standard deviation of the mean was calculated. Each of these values are listed in Table 3 , along with the observation date, orbital phase, and observed minus calculated values.

\subsection{Tomographic Reconstruction}

We used the Doppler tomography algorithm of Bagnuolo et al. (1994) to separate the primary and secondary spectra of HI Mon. This iterative method uses the eight observed composite spectra from outside eclipse phases, their observed velocity shifts, and an assumed monochromatic flux ratio to derive individual component spectra. The best flux ratio was the one that best matched the line depths in the reconstructions with those in the model spectra. Figure 3 shows the reconstructed spectra for the primary and secondary, along with the best fit model spectra for each. The region containing the diffuse interstellar absorption band around $\lambda 4428 \AA$, and other interstellar features were removed when performing the reconstruction, as otherwise this introduced noise into the final reconstructed spectra. Of particular importance are the fits of the wings of the $\mathrm{H} \gamma \lambda 4340$ line, as these are produced by linear Stark broadening and are good estimators for the gravities of the stars. The relative depths of the He I $\lambda 4471$ and Mg II $\lambda 4481$ lines give an indication of the temperature in early B-type stars. Note that fast rotation can lead to changing depths of these features, but these stars are not seen to be rotating quickly.

The final reconstructed spectra were fitted with TLUSTY/SYNSPEC model synthesis spectra (see Section 4.1). Estimates listed in Table 4 were made by comparing the reconstructed and model spectral line profiles against a grid of test values (see Williams et al. 2008; Williams 2009). Spectral type estimates were obtained by comparing the effective 
temperature against Table 2 of Böhm-Vitense (1981) and Table 3 of Harmaned (1988). The gravities of each star indicate they are main sequence objects, so final estimates for the spectral classifications are B0 $\mathrm{V}$ and B0.5 V.

\subsection{Combined Radial Velocity and Light-Curve Solution}

ELC was used to fit both the light curve and the radial velocity curves simultaneously. The parameters allowed to vary included the orbital period, epoch of primary eclipse, $T_{0}$, inclination, mass ratio, primary velocity semiamplitude, effective temperatures of each star, and Roche lobe filling factor for each star. The Roche lobe filling factor is defined by Orosz \& Hauschildt (2000) as the ratio of the radius of the star toward the inner Lagrangian point $\left(L_{1}\right)$ to the distance to $L_{1}$ from the center of the star, $f \equiv x_{\text {point }} / x_{\mathrm{L} 1}$. Nonzero eccentricities for HI Mon were explored during initial fitting, but rejected based on the higher $\chi^{2}$ values for those fits. HI Mon is therefore a "false member" of candidate eccentric systems listed in Hegedus (1988). The resulting best fit velocity curves are shown in Figure 4 and the best fit light curve is shown in Figure 5. During eclipses (at phase 0.0 and 0.5 in both Figures 4 and 5), bumps in the radial velocity curve can be seen due to the rotational Doppler shifts of the unobscured portions of the eclipsed star. This changes the shape of the observed spectral features, and gives rise to the velocity change, a perturbation known as the Rossiter-McLaughlin effect.

To estimate uncertainties based on our best fit, we used the nearly 40,000 recorded $\chi^{2}$ values for each fit to the light and radial velocity curves performed by ELC. We projected the well explored $\chi^{2}$ surface onto each parameter of interest. The lowest $\chi^{2}$ value is found for each parameter and the $1-\sigma$ uncertainty estimated from the region where $\chi^{2} \leq \chi_{\min }^{2}+1$. These values are listed in Table 5 for the circular orbital solution. Astrophysical parameters of interest are listed in Table 6 .

Comparing our results with those from the on-line catalog of Pilecki, Pojmański, \& Szczygieł reveals a few differences. Our inclination of $80.0 \pm 0.2$ agrees well with their inclination of 80.9. Also matching reasonably well are our temperature ratios, 0.963 from our best fit, and 0.971 in their catalog. Their estimations for polar radius of each star relative to its Roche lobe polar radius are 0.793 and 0.830 for the primary and secondary, respectively. These values are systematically larger than the values we derive from our best ELC fit, most likely due to the additional constraints from the spectra. The most discrepant set of values are for the temperatures of the individual stars. They list $8110 \mathrm{~K}$ for the primary and 7870 $\mathrm{K}$ for the secondary. They arrive at the value for the primary based on the $V-I$ color index. There are several problems with estimating the temperature of intermediate mass stars with 
only a color index. First, interstellar extinction has not been taken into account. Distances for mid to early B-type stars of the magnitude ranges covered in the ASAS $(V \sim 7-12)$ are going to be in excess of one kpc. Since these intermediate mass stars are typically found in or near the galactic plane, where extinction is more pronounced, any estimation of the temperature based on photometry will be effected by an unknown amount. Also, because the temperatures of intermediate mass binaries are high, their photometric colors sample the Rayleigh-Jeans tail of the energy distribution and the color differences are small. For example, the difference in $V-I$ is only $0.05 \mathrm{mag}$ between a $20 \mathrm{kK}$ star and a $25 \mathrm{kK}$ star (Bessell et al. 1998). Both of these factors illustrate the need for spectroscopy to understand the nature of these intermediate mass binaries.

\subsection{Discussion}

With a modest number of spectra (11 total), we have determined the masses and radii of each component in HI Mon to better than 3\% accuracy, as is seen in Table 6. Both stars are within their Roche lobes but experience tidal distortion, as evidenced in the light curve in Figure 5. Tidal evolution in the HI Mon system is also seen in the fact that the projected rotational velocities from the tomographic reconstructions (Table 4) and from the synchronous rates from ELC (Table 6) are consistent within uncertainties. This indicates that the system has had sufficient time to undergo tidal evolution in order to attain synchronous rotation.

To make a more quantitative estimate of the age of the system, we used the effective temperatures and effective radii of each component from Table 6 and plotted the two stars of HI Mon on a theoretical Hertzsprung-Russell (H-R) diagram to compare their locations with evolutionary tracks. Since the stars are tidally distorted, the effective radius of each star is the radius of a sphere with the same volume. Figure 6 shows evolutionary tracks from Schaller et al. (1992) for stars of 12 and $15 M_{\odot}$ and isochrones from Lejeune \& Schaerer (2001) for solar metallicity for ages of 1.5, 2.5, and 3.5 Myr. The ages of the stars are most consistent with an age of 2.5 Myr. The evolutionary tracks from Schaller et al. (1992) are for nonrotating stellar models. The ratios of spin angular velocity to critical angular velocity are $\Omega / \Omega_{\text {crit }}=0.42$ for the primary and 0.43 for the secondary. The evolutionary tracks for these moderate rotation rates are only slightly steeper and more extended in time than those illustrated (Ekström et al. 2008). Thus, our derived age is a lower limit.

We can also estimate the distance to the system by fitting a spectral energy distribution (SED) to photometric measurements from the literature (since our spectra were not flux calibrated). We used measurements from Vogt (1976) for $U, B$, and $V$ values, noting that 
the $V$-band measurement of maximum light from the ASAS light curve is consistent with these measures, meaning they were likely not taken during eclipse. In addition, we used 2MASS (Skrutskie et al. 2006) measurements to constrain the near-IR part of the SED. Strömgren photometric system measurements reported by Eggen (1978) were not consistent with the other photometry and were not used in the calculation of the SED. The SED fit is shown in Figure 7 with the $U, B, V, J, H$, and $K_{s}$ magnitudes transformed to fluxes using the calibrations of Colina et al. (1996) and Cohen et al. (2003). Model spectra for each star from Lanz \& Hubeny (2007) with parameters for our best fit from ELC (Table 6) were scaled in the blue by the flux ratio (also Table 6) of $0.81 \pm 0.09$ and added to form the total SED of the system. This fit of the SED results in a limb-darkened angular diameter for the primary of $\theta_{\mathrm{LD}}=21.5 \pm 0.7 \mu$ as and a reddening of $E(B-V)=0.35 \pm 0.02 \mathrm{mag}$ and a ratio of total-to-selective extinction of $R=3.2 \pm 0.1$. This reddening value matches reasonably well with the values in the literature of 0.30 (Eggen 1978) and 0.38 (Vogt 1976). Combining this angular diameter with the physical radius for the primary from Table 6 , we estimate the distance to HI Mon to be $2.26 \pm 0.04 \mathrm{kpc}$. By contrast, this value is not consistent with previous photometric distance estimates for HI Mon of $3.89 \mathrm{kpc}$ (Eggen 1978) and $3.98 \mathrm{kpc}$ (Vogt 1976).

HI Mon has galactic coordinates of $\ell=217.03$ and $b=-0.87$ (Reed 2005). The closest galactic cluster is NGC 2311 that is located at $\ell=217.76$ and $b=-0.69$ (Piatti et al. 2010). Piatti et al. (2010) find a distance to the cluster of $2.2 \pm 0.4 \mathrm{kpc}$ and a reddening of $E(B-V)=0.25 \pm 0.05$. However, they find an age for the cluster of $\sim 100 \mathrm{Myr}$, making HI Mon far too young to be associated with NGC 2311. The distance we find for HI Mon and its location suggest it is part of "Group A" as defined by Vogt (1976). This group is an association of luminous stars in the constellation of Monoceros at a distance of about 2.2 kpc that is part of the local arm of the galaxy.

This material is based on work supported by the National Science Foundation under grants AST-0606861 and AST-1009080. This publication makes use of data products from the Two Micron All Sky Survey, which is a joint project of the University of Massachusetts and the Infrared Processing and Analysis Center/California Institute of Technology, funded by the National Aeronautics and Space Administration and the National Science Foundation. This research has made use of the SIMBAD database, operated at CDS, Strasbourg, France.

Facility: Blanco 


\section{A. Light Curves of Intermediate Mass Binaries in the ASAS}

Figures of the light curves and fits for each of these systems are included in the on-line material as Figure Set A. These plots contain the ASAS data with uncertainties, phased to our derived orbits listed in Table 2. These best fit orbits are shown by green lines passing through the data between orbital phases -0.2 to 1.2 to show the primary eclipse in its entirety. There are several systems that have interesting features in their light curves. For example, Figure 26 shows the light curve for NSV 3433. It shows one of the larger temperature differences between components in this sample. It also shows an illumination effect on the secondary by the flux of the hotter primary. The out-of-eclipse flux is higher just before and after the secondary eclipse, indicating that the side of the secondary facing the primary is slightly hotter and giving off more light at this orbital phase. Figure 36 illustrates the light curve of HD 63818. This system has a moderately high eccentricity (0.132) with $\omega=343^{\circ}$, meaning the periastron of the orbit is close to the plane of the sky. This causes the secondary eclipse to be shifted from the circular case where it would be at 0.5 in orbital phase. A particularly illustrative example of this is TYC 1025-1524-1 seen in Figure 57. The widths of the eclipses are different owing to an eccentricity of 0.367 . Periastron occurs near phase $0.02\left(\omega=280^{\circ}\right)$, and the deeper, narrow eclipse at phase 0.0 results from the rapid and close passage of the secondary star in front of the primary star. Several systems have very well constrained light curves such as HD 48866 in Figure 16 and TT Pyx seen in Figure 40 that will make for highly accurate determinations of mass and radius when combined with double-lined spectroscopic orbits, and therefore, they represent the jewels of the collection.

\section{REFERENCES}

Bagnuolo, W. G., Jr., Gies, D. R., Hahula, M. E., Wiemker, R., \& Wiggs, M. S. 1994, ApJ, 423,446

Bessell, M. S., Castelli, F., \& Plez, B. 1998, A\&A, 333, 231

Bidelman, W. P. 1982, IBVS, 2112

Böhm-Vitense, E. 1981, ARA\&A, 19, 295

Brancewicz, H. K., \& Dworak, T. Z. 1980, Acta Astron., 30, 501

Cannon, A. J., \& Pickering, E. C. 1924, Annals of the Astronomical Observatory Henry Draper (HD) Catalog and HD Extension (Cambridge, MA: Harvard College)

Cohen, M., Wheaton, W. A., \& Megeath, S. T. 2003, AJ, 126, 1090 
Colina, L., Bohlin, R., \& Castelli, F. 1996, HST Instrument Science Report CAL-SCS-008 (Baltimore, MD: STScI)

Comerón, F., et al. 2002, A\&A, 389, 874

Deb, S., \& Singh, H. P. 2011, MNRAS, in press (astro-ph:1011.2574)

Eggen, O. J. 1978, AJ, 83, 288

Ekström, S., Meynet, G., Maeder, A., \& Barblan, F. 2008, A\&A, 478, 467

Gies, D. R., Penny, L. R., Mayer, P., Drechsel, H., \& Lorenz, R. 2002, ApJ, 574, 957

Giuricin, G., Mardirossian, F., \& Mezzetti, M. 1983, A\&AS, 54, 211

Harmanec, P. 1988, Bull. Astron. Inst. Cz., 39, 329

Heckmann, O. 1975, AGK3. Star catalogue of positions and proper motions north of -2.5 declination, ed. W. Dieckvoss (Hamburg-Bergedorf: Hamburger Sternwarte)

Hegedus, T. 1988, Bull. Inf. CDS, 35, 15

Hoffmeister, C. 1931, AN, 242, 129

Hoffmeister, C. 1933, AN, 247, 281

Hoffmeister, C. 1935, AN, 255, 401

Houk, N. 1982, Michigan Catalogue of Two-dimensional Spectral Types for the HD stars, Vol. 3. (Ann Arbor: Univ. of Michigan)

Houk, N., \& Smith-Moore, M. 1988, Michigan Catalogue of Two-dimensional Spectral Types for the HD Stars, Vol. 4, (Ann Arbor: Univ. of Michigan)

Houk, N., \& Swift, C. 1999, Michigan Catalogue of Two-dimensional Spectral Types for the HD Stars, Vol. 5 (Ann Arbor: Univ. of Michigan)

Hubeny, I. 1988, Computer Physics Communications, 52, 103

Hubeny, I., Heap, S. R., \& Lanz, T. 1998, in Boulder Munich II: Properties of Hot Luminous Stars (ASP Conf. Ser. Vol. 131) ed. I. D. Howarth (San Francisco: ASP), 108

Hubeny, I., \& Lanz, T. 1995, ApJ, 439, 875

Kazarovets, E. V., Samus, N. N., Durlevich, O. V., Frolov, M. S., Antipin, S. V., Kireeva, N. N., \& Pastukhova, E. N. 1999, IBVS, 4659 
Kenyon, S. J., \& Hartmann, L. 1995, ApJS, 101, 117

Lacy, C. H. S. 1993, AJ, 105, 637

Lanz, T., \& Hubeny, I. 2007, ApJS, 169, 83

Lejeune, T., \& Schaerer, D. 2001, A\&A, 366, 538

Meng, X., \& Yang, W. 2010, ApJ, 710, 1310

Milone, E. F. 1986, ApJS, 61, 455

Morgan, W. W., Code, A. D., \& Whitford, A. E. 1955, ApJS, 2, 41

Nesterov, V. V., Kuzmin, A. V., Ashimbaeva, N. T., Volchkov, A. A., Röser, S., \& Bastian, U. 1995, A\&AS, 110, 367

Ochsenbein, F. 1980, Bull. Inf. CDS, 19, 74

Orosz, J. A., \& Hauschildt, P. H. 2000, A\&A, 364, 265

Otero, S. A., Wils, P., \& Dubovsky, P. A. 2004, IBVS, 5570

Piatti, A. E., Clariá, J. J., \& Ahumada, A. V. 2010, MNRAS, 408, 1147

Pojmański, G. 2002, Acta Astron, 52, 397

Pojmański, G. 2003, Acta Astron, 53, 341

Pojmański, G., \& Maciejewski, G. 2004, Acta Astron, 54, 153

Pojmański, G., \& Maciejewski, G. 2005, Acta Astron, 55, 97

Pojmański, G., Pilecki, B., \& Szczygieł, D. 2005, Acta Astron, 55, 275

Poretti, E., et al. 2005, AJ, 129, 2461

Reed, B. C. 2005, AJ, 130, 1652

Schaller, G., Schaerer, D., Meynet, G., \& Maeder, A. 1992, A\&AS, 96, 269

Skrutskie, M. F., et al. 2006, AJ, 131, 1163

Strohmeier, W. 1966, IBVS, 158

Strohmeier, W., Knigge, R., \& Ott, H. 1965, IBVS, 107 
Struve, O. 1945, ApJ, 102, 74

Torres, G., Andersen, J., \& Giménez, A. 2010, A\&A Rev., 18, 67

Vogt, N. 1976, A\&A, 53, 9

Vogt, N., Kroll, P., \& Splittgerber, E. 2004, A\&A, 428, 925

Wachmann, A. A. 1964, Astron. Abh. Hamburg. Sternw., 7, 155

Wachmann, A. A. 1968, Astron. Abh. Hamburg. Sternw., 7, 381

Wallerstein, G. 1960, ApJ, 132, 37

Williams, S. J. 2009, AJ, 137, 3222

Williams, S. J., et al. 2008, ApJ, 682, 492

Wils, P., \& Dvorak, S. W. 2003, IBVS, 5425

Zinner, E. 1930, AN, 239, 59 
Table 1. Observational System Parameters

\begin{tabular}{|c|c|c|c|c|c|c|c|c|}
\hline $\begin{array}{c}\text { ASAS ID } \\
(1)\end{array}$ & $\begin{array}{c}\text { Other ID } \\
(2)\end{array}$ & $\begin{array}{c}\text { RA } \\
(\mathrm{J} 2000.0) \\
(3)\end{array}$ & $\begin{array}{c}\text { Declination } \\
\text { (J2000.0) } \\
(4)\end{array}$ & $\begin{array}{c}V-K \\
(\mathrm{mag}) \\
(5)\end{array}$ & $\begin{array}{c}Q \\
(\mathrm{mag}) \\
(6)\end{array}$ & $\begin{array}{c}\text { Spectral } \\
\text { Classification } \\
(7)\end{array}$ & $\begin{array}{c}\text { Classification } \\
\text { Reference } \\
(8)\end{array}$ & $\begin{array}{c}\text { Discovery } \\
\text { Reference } \\
\quad(9)\end{array}$ \\
\hline $053838+0901.2$ & HD 37396 & 053838.1 & +090110.7 & 0.33 & -0.10 & A0 & 1 & 14 \\
\hline $054816+2046.1$ & HD 247740 & 054816.5 & +204610.3 & 0.18 & -0.14 & B8 & 1 & ASAS \\
\hline $060857+1128.9$ & HD 252416 & $0608 \quad 57.2$ & +112556.2 & 0.19 & 0.00 & B8 & 1 & ASAS \\
\hline $060927-1501.7$ & TYC 5933-142-1 & 060926.6 & -150142.7 & 0.31 & -0.10 & $\ldots$ & $\cdots$ & ASAS \\
\hline $062556-1254.5$ & HD 45263 & 062556.1 & -125429.1 & 0.26 & -0.07 & B9 IV & 2 & ASAS \\
\hline $063347-1410.5$ & HD 46621 & 063346.8 & -141030.9 & 0.28 & 0.14 & $\mathrm{~A} 1 \mathrm{IV} / \mathrm{V}$ & 2 & ASAS \\
\hline $064010-1140.3$ & HD 47845 & 064009.4 & $\begin{array}{lll}-11 & 40 & 17.8\end{array}$ & 0.47 & 0.05 & A2 IV & 3 & ASAS \\
\hline $064118-0551.1$ & 2MASS06411762-0551065 & 064117.6 & -055106.9 & 0.43 & -0.04 & $\ldots$ & $\ldots$ & ASAS \\
\hline $064538+0219.4$ & HD 48866 & 064537.8 & +02 1921.1 & -0.04 & -0.10 & $\mathrm{~A}(\mathrm{~V})+\mathrm{A}(\mathrm{V})$ & 3 & 15 \\
\hline $064609-1923.8$ & HD 49125 & 064609.4 & -192350.1 & -0.03 & -0.08 & B9 V & 2 & ASAS \\
\hline $064715+0225.6$ & HD 289072 & 064714.6 & +022534.3 & 0.37 & -0.03 & B5 & 4 & ASAS \\
\hline $064745+0122.3$ & V448 Mon & 064745.0 & +012218.0 & 0.14 & 0.09 & B5 & 4 & 16 \\
\hline $065534-1013.2$ & HD 51082 & 065533.9 & -101312.6 & 0.02 & -0.15 & A0 V & 3 & 17 \\
\hline $065549-0402.6$ & HI Mon & 065549.1 & -040235.8 & 0.22 & -0.04 & $\mathrm{~B} 3 / 5 \mathrm{II}$ & 3 & 18 \\
\hline $070105-0358.2$ & HD 52433 & 070105.1 & -035815.5 & -0.17 & 0.02 & B3 III & 3 & 19 \\
\hline $070238+1347.0$ & HD 52637 & 070238.2 & +134658.6 & 0.23 & 0.03 & A0 & 1 & 20 \\
\hline $070636-0437.4$ & AO Mon & 070636.3 & -043724.5 & -0.43 & -0.04 & $\mathrm{~B} 3+\mathrm{B} 5$ & 5 & 21 \\
\hline $070943+2341.7$ & $\mathrm{BD}+231621$ & 070943.3 & +234142.8 & 0.70 & 0.01 & $\ldots$ & $\ldots$ & ASAS \\
\hline $070946-2005.5$ & NSV 3433 & 070946.2 & -200535.2 & -0.09 & 0.01 & $\ldots$ & $\ldots$ & 17 \\
\hline $071010-0035.1$ & HD 54780 & 071010.4 & -003508.7 & 0.22 & -0.17 & B5/7 (III) & 3 & 15 \\
\hline $071203-0139.1$ & HD 55236 & 071203.5 & -013904.2 & 0.54 & 0.04 & A2 III/IV & 3 & ASAS \\
\hline $071702-1034.9$ & HD $56544^{\mathrm{a}}$ & $07 \quad 1702.4$ & -103456.6 & -0.30 & 0.02 & A0 & 1 & ASAS \\
\hline $072201-2552.6$ & $\mathrm{CX} \mathrm{CMa}$ & 072201.0 & -255235.9 & -0.30 & -0.10 & B5 V & 6 & 21 \\
\hline $073053+0513.7$ & HD 59607 & 073053.5 & +051337.3 & -0.27 & -0.11 & B8 & 1 & ASAS \\
\hline $073348-0940.9$ & HD 60389 & 073348.4 & -094052.6 & 0.45 & -0.02 & G6 V & 3 & 22 \\
\hline $074355-2517.9$ & HD 62607 & 074355.2 & -251753.7 & 0.08 & -0.08 & B9.5 V & 2 & ASAS \\
\hline $074717-0519.8$ & HD 63141 & $0747 \quad 17.3$ & -051951.1 & 0.29 & -0.01 & $\mathrm{~A} 0 \mathrm{~V}$ & 3 & 17 \\
\hline $074928-0721.6$ & $\mathrm{BD}-062317$ & 074927.5 & -072136.0 & 0.23 & -0.05 & A0 & 7 & ASAS \\
\hline $075052+0048.0$ & HD 63818 & 075052.4 & +004804.1 & -0.06 & -0.07 & $\mathrm{~A} 0$ & 1 & ASAS \\
\hline $080617-0426.8$ & V871 Mon & $\begin{array}{lll}08 & 06 & 17.3\end{array}$ & -042646.8 & 0.64 & 0.02 & $\mathrm{~A} 3 / 5 \mathrm{~mA} 7-\mathrm{F} 0$ & 3 & 23 \\
\hline $081749-2659.7$ & HD 69797 & $\begin{array}{lll}08 & 17 & 48.7\end{array}$ & -265937.5 & 0.66 & -0.22 & $\mathrm{~A} 1 \mathrm{~V}$ & 8 & ASAS \\
\hline $083245+0247.3$ & $\mathrm{BD}+032001$ & 083245.3 & +024716.5 & 0.79 & 0.07 & A2 & 9 & ASAS \\
\hline $084831-2609.8$ & TT Pyx & 084830.9 & -260947.8 & 0.16 & -0.08 & B9.5 IV & 2 & 24 \\
\hline
\end{tabular}


Table 1-Continued

\begin{tabular}{|c|c|c|c|c|c|c|c|c|}
\hline $\begin{array}{c}\text { ASAS ID } \\
(1)\end{array}$ & $\begin{array}{l}\text { Other ID } \\
(2)\end{array}$ & $\begin{array}{c}\text { RA } \\
(\mathrm{J} 2000.0) \\
(3)\end{array}$ & $\begin{array}{c}\text { Declination } \\
(\mathrm{J} 2000.0) \\
(4)\end{array}$ & $\begin{array}{c}V-K \\
(\mathrm{mag}) \\
(5)\end{array}$ & $\begin{array}{c}Q \\
(\mathrm{mag}) \\
(6)\end{array}$ & $\begin{array}{c}\text { Spectral } \\
\text { Classification } \\
(7)\end{array}$ & $\begin{array}{c}\text { Classification } \\
\text { Reference } \\
\text { (8) }\end{array}$ & $\begin{array}{c}\text { Discovery } \\
\text { Reference } \\
\quad(9)\end{array}$ \\
\hline $101120-1956.3$ & HD 88409 & 101119.5 & -195620.4 & 0.49 & 0.04 & A5 II/III & 2 & ASAS \\
\hline $135949-2745.5$ & HD 122026 & 135949.3 & -274526.4 & 0.52 & 0.03 & A1/2 III/IV & 8 & ASAS \\
\hline $160851-2351.0$ & TYC $6780-1523-1$ & 160851.0 & -235101.4 & 1.56 & 0.09 & $\ldots$ & $\cdots$ & ASAS \\
\hline $165354-1301.9$ & HD 152451 & 165354.2 & -130157.5 & 1.85 & 0.14 & A9 V & 2 & ASAS \\
\hline $170158+2348.4$ & HD 154010 & 170157.5 & +234822.5 & 0.56 & -0.01 & A2 & 1 & ASAS \\
\hline $173421-1836.3$ & HD 159246 & 173421.1 & -183621.7 & 0.57 & -0.08 & B9 III & 2 & ASAS \\
\hline $174104+0747.1$ & V506 Oph & 174104.3 & +074704.3 & 1.12 & 0.10 & A9 & 10 & 25 \\
\hline $175659-2012.2$ & HD 312444 & 175658.8 & -201205.7 & 1.46 & -0.04 & A3 & 3 & ASAS \\
\hline $175859-2323.1$ & HD 313508 & 175859.3 & -232307.9 & 0.68 & -0.76 & B8 & 3 & ASAS \\
\hline $180903-1824.5$ & HD 165890 & 180902.9 & -182429.5 & 0.66 & -0.01 & $\mathrm{~B} 7 / 8 \mathrm{Ib}$ & 2 & ASAS \\
\hline $181025+0047.7$ & HD 166383 & 181025.7 & +004747.0 & 0.88 & 0.15 & B3/5 II/III & 3 & ASAS \\
\hline $181328-2214.3$ & HD 166851 & 181328.0 & -221407.2 & 0.67 & -0.03 & B9 III/IV & 2 & ASAS \\
\hline $181909-1410.0$ & HD 168207 & 181909.0 & -141000.6 & 1.05 & 0.05 & $\mathrm{~B} 1 / 2(\mathrm{~N})$ & 2 & ASAS \\
\hline $183129-1918.8$ & BD -19 5039 & 183128.9 & -191847.3 & 0.90 & 0.02 & B3 V & 11 & ASAS \\
\hline $183219-1117.4$ & $\mathrm{BD}-114667$ & 183219.0 & -111723.6 & 2.21 & 0.02 & B1:V:pe & 12 & 26 \\
\hline $184223+1158.9$ & $\mathrm{BD}+113569$ & 184223.2 & +115857.4 & -0.24 & 0.01 & A2 & 9 & ASAS \\
\hline $184327+0841.5$ & TYC $1025-1524-1$ & 184326.9 & +084132.1 & 0.53 & 0.00 & $\ldots$ & $\cdots$ & ASAS \\
\hline $184436-1923.4$ & YY Sgr & 184435.9 & -192322.7 & 0.41 & 0.12 & $\mathrm{~B} 5+\mathrm{B} 6$ & 13 & 27 \\
\hline $185051-1354.6$ & HD 174397 & 185051.3 & -135433.1 & 1.40 & 0.01 & A9 & 2 & ASAS \\
\hline $194334-0904.0$ & V1461 Aql & 194334.1 & -090402.1 & 1.17 & 0.19 & A0 III & 3 & 20 \\
\hline $195342+0205.4$ & HD 188153 & 195342.0 & +020521.2 & 0.32 & -0.01 & A0 & 1 & ASAS \\
\hline $195613+1630.9$ & HD 354110 & 195613.1 & +163059.1 & 0.19 & -0.01 & B8 & 3 & ASAS \\
\hline $205642+1153.0$ & HD 199428 & 205642.0 & +115302.3 & 0.54 & -0.02 & $\mathrm{~A} 2$ & 1 & ASAS \\
\hline
\end{tabular}

References: 1-Cannon \& Pickering (1924), 2-Houk \& Smith-Moore (1988), 3-Houk \& Swift (1999), 4-Nesterov et al. (1995), 5- Struve (1945), 6- Milone (1986),

7-Ochsenbein (1980), 8-Houk (1982), 9-Heckmann (1975), 10-Brancewicz \& Dworak (1980), 11-Wallerstein (1960), 12-Morgan et al. (1955), 13-Lacy (1993),

14-Vogt et al. (2004), 15-Poretti et al. (2005), 16-Wachmann (1964), 17-Strohmeier et al. (1965), 18-Wachmann (1968), 19-Otero et al. (2004),

20-Kazarovets et al. (1999), 21-Hoffmeister (1931), 22-Strohmeier (1966), 23-Wils \& Dvorak (2003), 24-Hoffmeister (1933), 25-Hoffmeister (1935),

26-Bidelman (1982), 27-Zinner (1930) 
Table 2. Derived System Parameters

\begin{tabular}{|c|c|c|c|c|c|c|}
\hline $\begin{array}{c}\text { ASAS ID } \\
(1)\end{array}$ & $\begin{array}{c}\text { Other ID } \\
(2)\end{array}$ & $\begin{array}{l}P \\
(\mathrm{~d}) \\
(3)\end{array}$ & $\begin{array}{c}T_{0} \\
(\mathrm{HJD}-2,400,000) \\
(4)\end{array}$ & $\begin{array}{c}i \\
\left({ }^{\circ}\right) \\
(5)\end{array}$ & $\begin{array}{c}e \\
(6)\end{array}$ & $\begin{array}{l}\omega \\
\left({ }^{\circ}\right) \\
(7)\end{array}$ \\
\hline $053838+0901.2$ & HD 37396 & $1.22667(1)$ & $54900.712(1)$ & $77.5(8)$ & 0.0 & $\cdots$ \\
\hline $054816+2046.1$ & HD 247740 & $2.43242(2)$ & $54882.262(1)$ & $82.5(2)$ & 0.0 & $\cdots$ \\
\hline $060857+1128.9$ & HD 252416 & $1.9064700(1)$ & $54937.313(1)$ & $80.2(3)$ & 0.0 & $\cdots$ \\
\hline $060927-1501.7$ & TYC 5933-142-1 & $0.8770820(3)$ & $54900.6813(5)$ & $75.8(2)$ & 0.0 & $\cdots$ \\
\hline $062556-1254.5$ & HD 45263 & $5.059500(4)$ & $54952.100(1)$ & $89.6(3)$ & 0.0 & $\cdots$ \\
\hline $063347-1410.5$ & HD 46621 & $1.5958525(7)$ & $54953.6023(5)$ & $84.3(6)$ & 0.0 & $\cdots$ \\
\hline $064010-1140.3$ & HD 47845 & $5.285085(4)$ & $53847.994(2)$ & $88.2(1)$ & 0.0 & $\cdots$ \\
\hline $064118-0551.1$ & 2MASS06411762-0551065 & $2.757425(6)$ & $54931.327(2)$ & $79.2(3)$ & 0.0 & $\cdots$ \\
\hline $064538+0219.4$ & HD 48866 & $1.568196(2)$ & $54905.362(2)$ & $74.2(3)$ & 0.0 & $\ldots$ \\
\hline $064609-1923.8$ & HD 49125 & $2.9038975(3)$ & $54908.0579(1)$ & $88.6(1)$ & $0.050(2)$ & $53.1(2)$ \\
\hline $064715+0225.6$ & HD 289072 & $3.47257(1)$ & $54931.700(3)$ & $76.1(3)$ & $0.035(20)$ & $124(9)$ \\
\hline $064745+0122.3$ & V448 Mon & $1.118478(2)$ & $54933.555(1)$ & $74.6(7)$ & 0.0 & $\ldots$ \\
\hline $065534-1013.2$ & HD 51082 & $2.186885(3)$ & $54586.2521(8)$ & $81.2(2)$ & 0.0 & $\ldots$ \\
\hline $065549-0402.6$ & HI Mon & $1.5744300(8)$ & $54935.531(1)$ & $80.1(2)$ & 0.0 & $\ldots$ \\
\hline $070105-0358.2$ & HD 52433 & $5.95576(2)$ & $54603.120(3)$ & $77.8(1)$ & $0.067(8)$ & $54(3)$ \\
\hline $070238+1347.0$ & HD 52637 & $1.918694(4)$ & $54809.538(3)$ & $75.1(3)$ & 0.0 & $\cdots$ \\
\hline $070636-0437.4$ & AO Mon & $1.8847476(1)$ & $54965.2835(5)$ & $85.5(1)$ & $0.027(2)$ & $78(3)$ \\
\hline $070943+2341.7$ & $\mathrm{BD}+231621$ & $1.67610(1)$ & $54944.095(2)$ & $72.5(2)$ & 0.0 & $\ldots$ \\
\hline $070946-2005.5$ & NSV 3433 & $1.7568950(1)$ & $55002.5074(6)$ & $85.2(7)$ & 0.0 & $\ldots$ \\
\hline $071010-0035.1$ & HD 54780 & $2.228760(8)$ & $54963.204(2)$ & $71.8(3)$ & 0.0 & $\ldots$ \\
\hline $071203-0139.1$ & HD 55236 & $1.2834150(5)$ & $54944.918(1)$ & $78.0(3)$ & 0.0 & $\ldots$ \\
\hline $071702-1034.9$ & HD 56544 & $1.276465(2)$ & $55001.2242(7)$ & $76.6(5)$ & 0.0 & $\ldots$ \\
\hline $072201-2552.6$ & CX CMa & $0.95462500(2)$ & $55007.3938(7)$ & $81.8(2)$ & 0.0 & $\cdots$ \\
\hline $073053+0513.7$ & HD 59607 & $4.13935(3)$ & $54962.503(4)$ & $84(2)$ & 0.0 & $\ldots$ \\
\hline $073348-0940.9$ & HD 60389 & $2.549608(5)$ & $54573.822(2)$ & $76.4(3)$ & 0.0 & $\cdots$ \\
\hline $074355-2517.9$ & HD 62607 & $3.863847(9)$ & $54606.582(2)$ & $82.5(4)$ & 0.0 & $\ldots$ \\
\hline $074717-0519.8$ & HD 63141 & $4.593955(8)$ & $54958.149(4)$ & $85.2(1)$ & 0.0 & $\cdots$ \\
\hline $074928-0721.6$ & $\mathrm{BD}-062317$ & $2.152188(1)$ & $54966.082(1)$ & $76.2(3)$ & 0.0 & $\ldots$ \\
\hline $075052+0048.0$ & HD 63818 & $2.0543180(7)$ & $54966.584(2)$ & $80.2(2)$ & $0.132(1)$ & $343(3)$ \\
\hline $080617-0426.8$ & V871 Mon & $4.335950(3)$ & $54962.888(1)$ & $82.6(2)$ & $0.023(9)$ & $272(5)$ \\
\hline $081749-2659.7$ & HD 69797 & $1.743810(5)$ & $54931.5934(6)$ & $86.9(5)$ & 0.0 & $\ldots$ \\
\hline $083245+0247.3$ & $\mathrm{BD}+032001$ & $1.657373(2)$ & $54937.461(1)$ & $78.7(2)$ & 0.0 & $\cdots$ \\
\hline $084831-2609.8$ & TT Pyx & $3.031555(1)$ & $54987.7396(7)$ & $89.5(5)$ & 0.0 & $\ldots$ \\
\hline $101120-1956.3$ & HD 88409 & $1.563820(3)$ & $54902.837(1)$ & $77.8(7)$ & 0.0 & $\cdots$ \\
\hline $135949-2745.5$ & HD 122026 & $6.133165(7)$ & $55002.2808(9)$ & $88.2(6)$ & 0.0 & $\ldots$ \\
\hline $160851-2351.0$ & TYC $6780-1523-1$ & $4.66007(1)$ & $54801.465(3)$ & $81.9(4)$ & 0.0 & $\ldots$ \\
\hline $165354-1301.9$ & HD 152451 & $2.207586(1)$ & $54804.5134(4)$ & $83.1(2)$ & $0.215(3)$ & $157(2)$ \\
\hline $170158+2348.4$ & HD 154010 & $4.76991(1)$ & $54700.588(2)$ & $86.2(2)$ & 0.0 & $\ldots$ \\
\hline $173421-1836.3$ & HD 159246 & $5.216895(4)$ & $54807.627(1)$ & $89.8(3)$ & 0.0 & $\cdots$ \\
\hline $174104+0747.1$ & V506 Oph & $1.060427(1)$ & $54806.6804(4)$ & $88.5(6)$ & 0.0 & $\ldots$ \\
\hline $175659-2012.2$ & HD 312444 & $3.089365(5)$ & $54803.486(1)$ & $82.8(4)$ & 0.0 & $\cdots$ \\
\hline $175859-2323.1$ & HD 313508 & $1.540495(5)$ & $55006.029(1)$ & $79.0(6)$ & 0.0 & $\ldots$ \\
\hline $180903-1824.5$ & HD 165890 & $3.70752(1)$ & $54807.316(5)$ & $80.9(3)$ & 0.0 & $\cdots$ \\
\hline $181025+0047.7$ & HD 166383 & $3.18867(1)$ & $54808.146(7)$ & $71.7(3)$ & 0.0 & $\ldots$ \\
\hline
\end{tabular}


Table 2-Continued

\begin{tabular}{|c|c|c|c|c|c|c|}
\hline $\begin{array}{c}\text { ASAS ID } \\
(1)\end{array}$ & $\begin{array}{c}\text { Other ID } \\
(2)\end{array}$ & $\begin{array}{l}P \\
\text { (d) } \\
(3)\end{array}$ & $\begin{array}{c}T_{0} \\
(\mathrm{HJD}-2,400,000) \\
(4)\end{array}$ & $\begin{array}{c}i \\
\left(\begin{array}{l}\circ \\
(5)\end{array}\right.\end{array}$ & $\begin{array}{c}e \\
(6)\end{array}$ & $\begin{array}{l}\omega \\
\left({ }^{\circ}\right) \\
(7)\end{array}$ \\
\hline $181328-2214.3$ & HD 166851 & $4.47977(3)$ & $54803.532(14)$ & $88(2)$ & 0.0 & $\cdots$ \\
\hline $181909-1410.0$ & HD 168207 & $1.786820(1)$ & $54803.012(2)$ & $70.1(2)$ & 0.0 & $\cdots$ \\
\hline $183129-1918.8$ & BD -19 5039 & $2.455005(6)$ & $54949.211(2)$ & $74.7(3)$ & 0.0 & $\cdots$ \\
\hline $183219-1117.4$ & BD -114667 & $4.95239(2)$ & $55038.863(5)$ & $83.1(9)$ & 0.0 & $\ldots$ \\
\hline $184223+1158.9$ & $\mathrm{BD}+113569$ & $1.497118(3)$ & $55008.3686(9)$ & $81.1(4)$ & $0.006(2)$ & $144(31)$ \\
\hline $184327+0841.5$ & TYC $1025-1524-1$ & $2.1392200(8)$ & $55049.120(1)$ & $84.6(1)$ & $0.367(5)$ & $280.0(2)$ \\
\hline $184436-1923.4$ & YY Sgr & $2.6284575(6)$ & $55026.2658(5)$ & 88.1(1) & $0.117(1)$ & $214.1(5)$ \\
\hline $185051-1354.6$ & HD 174397 & $3.145935(8)$ & $55047.304(5)$ & $78.0(7)$ & 0.0 & $\ldots$ \\
\hline $194334-0904.0$ & V1461 Aql & $1.76306(1)$ & $55040.5159(8)$ & $82.4(5)$ & 0.0 & $\ldots$ \\
\hline $195342+0205.4$ & HD 188153 & $1.608120(2)$ & $55040.431(2)$ & $73.0(2)$ & $0.040(2)$ & $27(3)$ \\
\hline $195613+1630.9$ & HD 354110 & $3.444589(5)$ & $55036.802(3)$ & $85.5(1)$ & $0.037(29)$ & $102(2)$ \\
\hline $205642+1153.0$ & HD 199428 & $4.124500(5)$ & $55047.514(1)$ & $86.2(4)$ & $0.219(1)$ & $328.2(2)$ \\
\hline
\end{tabular}


Table 3. HI Mon Radial Velocity Measurements

\begin{tabular}{cccccccc}
\hline \hline $\begin{array}{c}\text { Date } \\
(\text { HJD-2,400,000) }\end{array}$ & $\begin{array}{c}\text { Orbital } \\
\text { Phase }\end{array}$ & $\begin{array}{c}V_{1} \\
\left(\mathrm{~km} \mathrm{~s}^{-1}\right)\end{array}$ & $\begin{array}{c}\sigma_{1} \\
\left(\mathrm{~km} \mathrm{~s}^{-1}\right)\end{array}$ & $\begin{array}{c}(O-C)_{1} \\
\left(\mathrm{~km} \mathrm{~s}^{-1}\right)\end{array}$ & $\begin{array}{c}V_{2} \\
\left(\mathrm{~km} \mathrm{~s}^{-1}\right)\end{array}$ & $\begin{array}{c}\sigma_{2} \\
\left(\mathrm{~km} \mathrm{~s}^{-1}\right)\end{array}$ & $\begin{array}{c}(O-C)_{2} \\
\left(\mathrm{~km} \mathrm{~s}^{-1}\right)\end{array}$ \\
\hline 55190.776 & 0.119 & -90.2 & 4.5 & 15.7 & 257.3 & 2.4 & 3.0 \\
55190.837 & 0.157 & -131.9 & 4.9 & 11.9 & 306.7 & 7.2 & 8.0 \\
55191.769 & 0.750 & 312.2 & 8.2 & 0.8 & -224.0 & 7.1 & 1.9 \\
55191.818 & 0.780 & 306.7 & 8.2 & -0.8 & -219.4 & 8.2 & 1.2 \\
55191.846 & 0.798 & 298.4 & 7.9 & -2.8 & -211.1 & 8.5 & 1.8 \\
55192.648 & 0.308 & -166.6 & 2.6 & -0.3 & 329.9 & 3.8 & 1.7 \\
55192.680 & 0.328 & -157.6 & 2.2 & -4.1 & 311.5 & 4.8 & -2.1 \\
55192.797 & 0.402 & -82.2 & 3.6 & -3.9 & 221.1 & 4.1 & -5.8 \\
55192.838 & 0.429 & -52.1 & 5.4 & -9.2 & 185.7 & 6.5 & -17.0 \\
55193.777 & 0.024 & -0.9 & 13.5 & 17.3 & 107.4 & 12.4 & 3.6 \\
55193.828 & 0.057 & -40.4 & 7.8 & 12.0 & 156.4 & 8.2 & -4.6 \\
\hline
\end{tabular}

Table 4. Tomographic Spectral Reconstruction Parameters

\begin{tabular}{ccc}
\hline \hline Parameter & Primary & Secondary \\
\hline Spectral Type $^{\mathrm{a}}$. & $\mathrm{B} 0 \mathrm{~V}$ & $\mathrm{~B} 0.5 \mathrm{~V}$ \\
$T_{\text {eff }}(\mathrm{kK}) \ldots \ldots$ & $30.0 \pm 0.5$ & $29.0 \pm 0.5$ \\
$\log g(\mathrm{cgs}) \ldots \ldots$ & $4.1 \pm 0.2$ & $4.1 \pm 0.2$ \\
$V \sin i\left(\mathrm{~km} \mathrm{~s}^{-1}\right)$ & $150 \pm 25$ & $150 \pm 25$ \\
$F_{2} / F_{1}$ (blue) & $0.70 \pm 0.05$ \\
\hline
\end{tabular}

aThese spectral types are estimated from derived values of $T_{\text {eff }}$ and $\log g$. 
Table 5. Circular Orbital Solution for HI Mon

\begin{tabular}{|c|c|}
\hline Element & Value \\
\hline$P($ days $)$. & $1.5744300 \pm 0.0000008$ \\
\hline$T_{0}$ (HJD-2,400,000) $\ldots$. & $54935.5331 \pm 0.0005$ \\
\hline$K_{1}\left(\mathrm{~km} \mathrm{~s}^{-1}\right) \ldots \ldots \ldots \ldots$ & $248.8 \pm 1.9$ \\
\hline$K_{2}\left(\mathrm{~km} \mathrm{~s}^{-1}\right) \ldots \ldots \ldots \ldots$ & $288.2 \pm 2.4$ \\
\hline$\gamma_{1}\left(\mathrm{~km} \mathrm{~s}^{-1}\right) \ldots \ldots \ldots \ldots$ & $64.5 \pm 1.3$ \\
\hline$\gamma_{2}\left(\mathrm{~km} \mathrm{~s}^{-1}\right) \ldots \ldots \ldots \ldots$ & $59.8 \pm 1.2$ \\
\hline rms (primary) $\left(\mathrm{km} \mathrm{s}^{-1}\right)$ & 9.6 \\
\hline rms (secondary) $\left(\mathrm{km} \mathrm{s}^{-1}\right)$ & 6.5 \\
\hline rms (photometry) (mag). & 0.02 \\
\hline
\end{tabular}

Table 6. ELC Model Parameters for HI Mon

\begin{tabular}{ccc}
\hline \hline Parameter & Primary & Secondary \\
\hline Inclination $(\mathrm{deg}) \ldots$ & \multicolumn{2}{c}{$80.0 \pm 0.2$} \\
$M\left(M_{\odot}\right) \ldots \ldots \ldots \ldots$ & $14.2 \pm 0.3$ & $12.2 \pm 0.2$ \\
$R_{\text {eff }}\left(R_{\odot}\right) \ldots \ldots \ldots$ & $5.13 \pm 0.11$ & $4.99 \pm 0.07$ \\
$R_{\text {pole }}{ }^{\mathrm{a}}\left(R_{\odot}\right) \ldots \ldots \ldots$ & $4.99 \pm 0.11$ & $4.83 \pm 0.07$ \\
$R_{\text {point }}{ }^{\mathrm{b}}\left(R_{\odot}\right) \ldots \ldots$ & $5.40 \pm 0.11$ & $5.30 \pm 0.07$ \\
$V_{\text {sync }} \sin i\left(\mathrm{~km} \mathrm{~s}^{-1}\right)$ & $162.5 \pm 7.2$ & $157.9 \pm 2.7$ \\
$\log g(\operatorname{cgs}) \ldots \ldots \ldots$ & $4.17 \pm 0.04$ & $4.13 \pm 0.04$ \\
$T_{\text {eff }}(\mathrm{kK}) \ldots \ldots \ldots \ldots$ & $29.5 \pm 0.6$ & $28.4 \pm 0.4$ \\
Filling factor $\ldots \ldots$ & $0.62 \pm 0.01$ & $0.65 \pm 0.02$ \\
$a_{\text {tot }}\left(R_{\odot}\right) \ldots \ldots \ldots$ & $16.96 \pm 0.11$ \\
$F_{2} / F_{1}($ blue $) \ldots \ldots$ & $0.81 \pm 0.09$ \\
\hline
\end{tabular}

${ }^{\text {aPolar radius. }}$

${ }^{\mathrm{b}}$ Radius toward the inner Lagrangian point. 


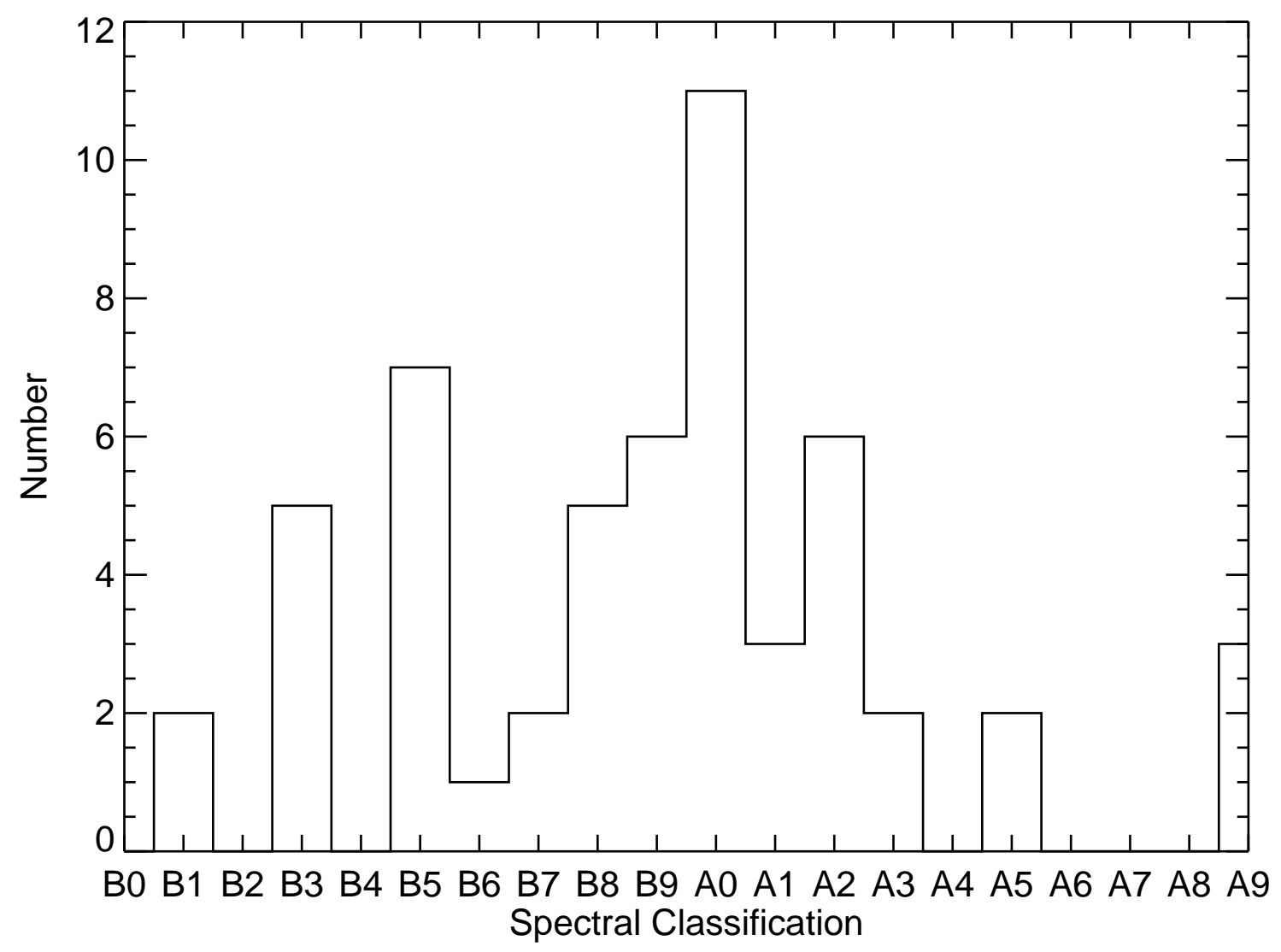

Fig. 1.- A histogram of published spectral types for our target objects. 


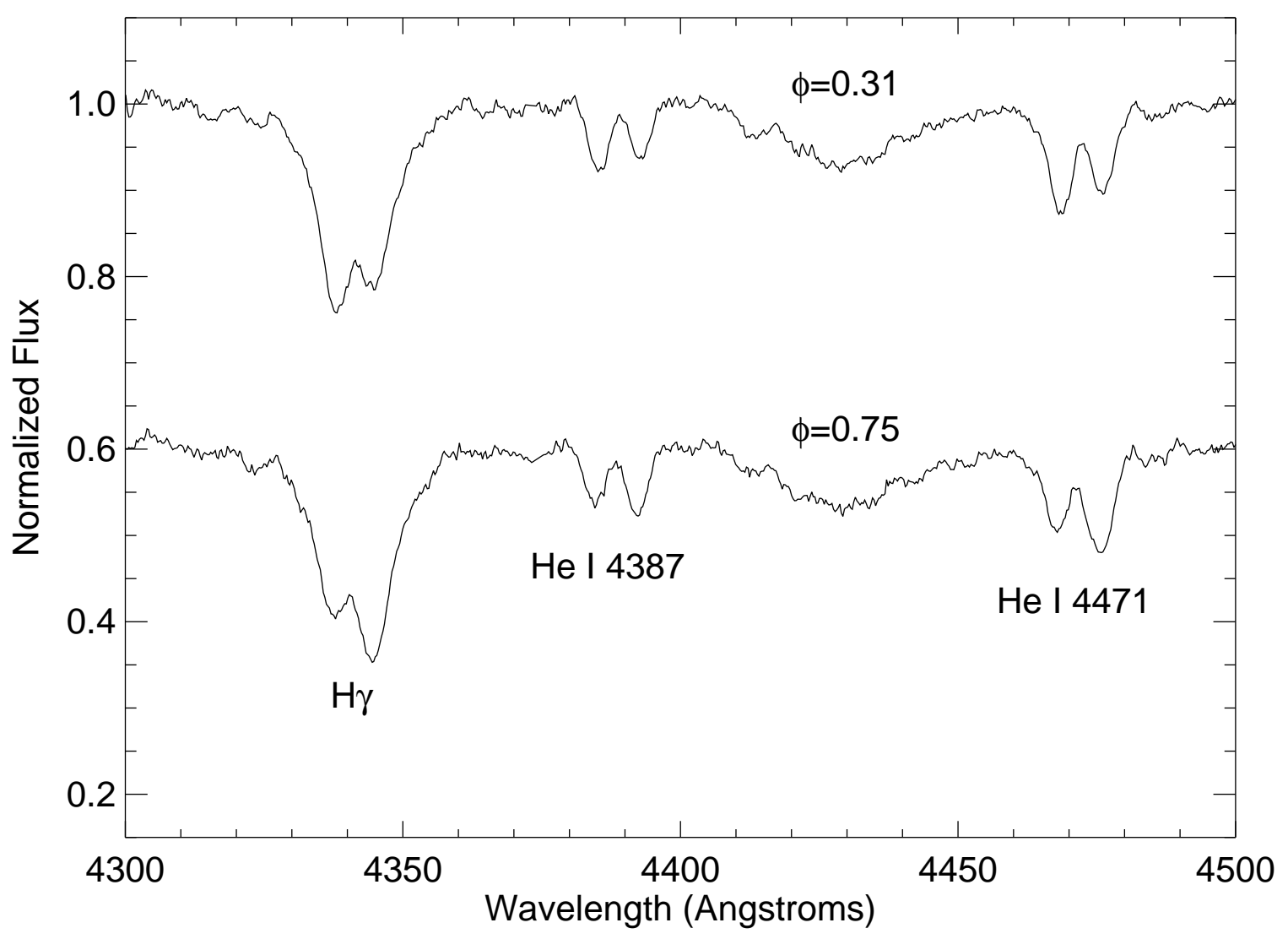

Fig. 2.- Two spectra of HI Mon near opposing quadrature phases (offset for clarity). The primary in each spectrum is represented by the deeper lines. 


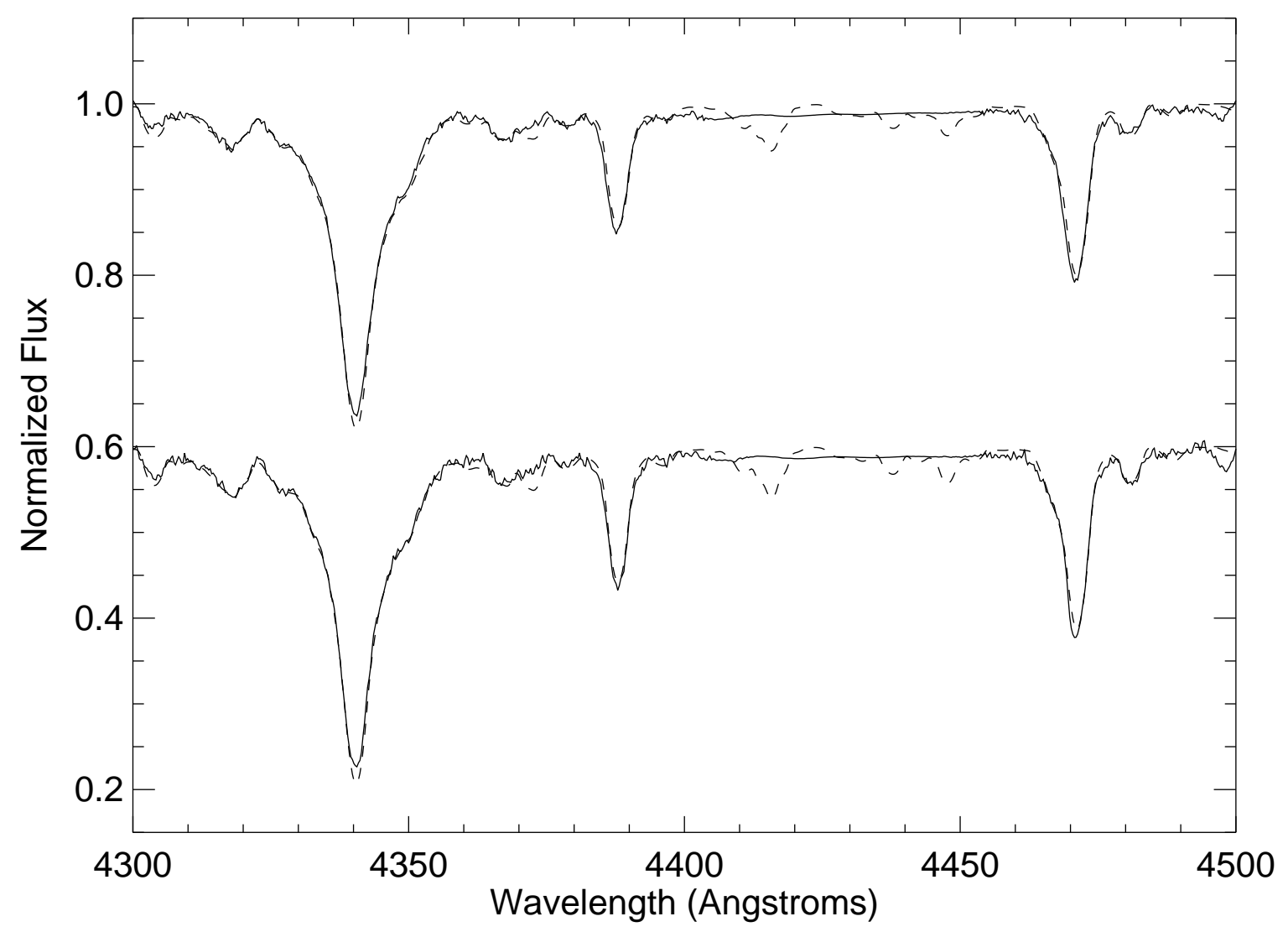

Fig. 3.- Tomographic reconstructions of the components of HI Mon based on the 9 spectra obtained outside of eclipse phases. The top solid line represents the primary and the bottom solid line is the reconstructed secondary spectrum offset by 0.4 for clarity. Overplotted for both are the model spectra for each shown by dashed lines. The stellar parameters for the model spectra are given in Table 4. Note also that the region containing the diffuse interstellar band near $4428 \AA$ has been removed for the tomographic reconstruction. 


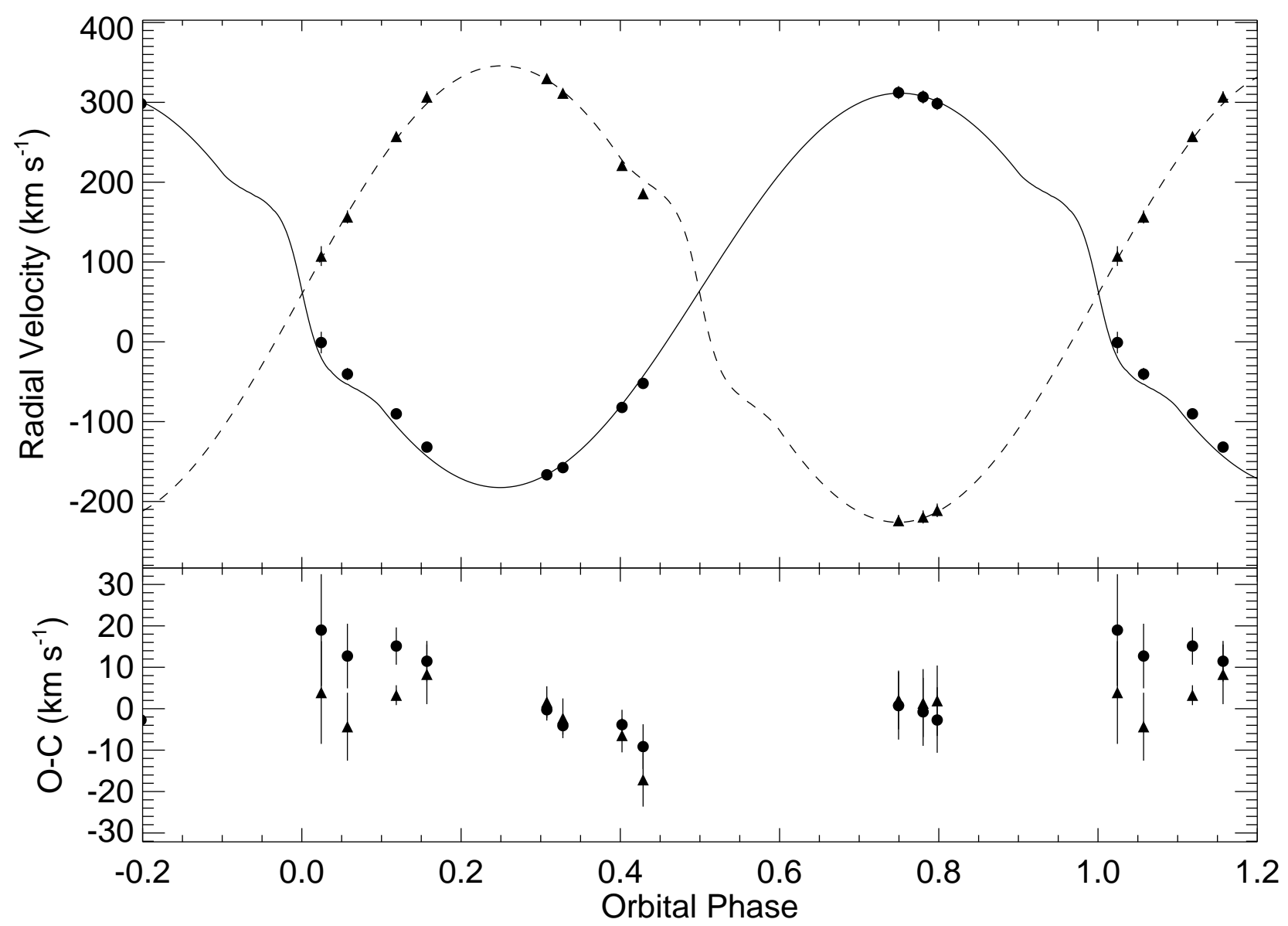

Fig. 4. - Radial velocity curves for HI Mon. Primary radial velocities are represented by filled dots and secondary radial velocities by filled triangles with associated uncertainties shown as line segments for both. The solid line is the best-fit solution for the primary and the dashed line is the best-fit solution for the secondary. The lower panel shows the observed minus calculated values for each measurement with uncertainties. 


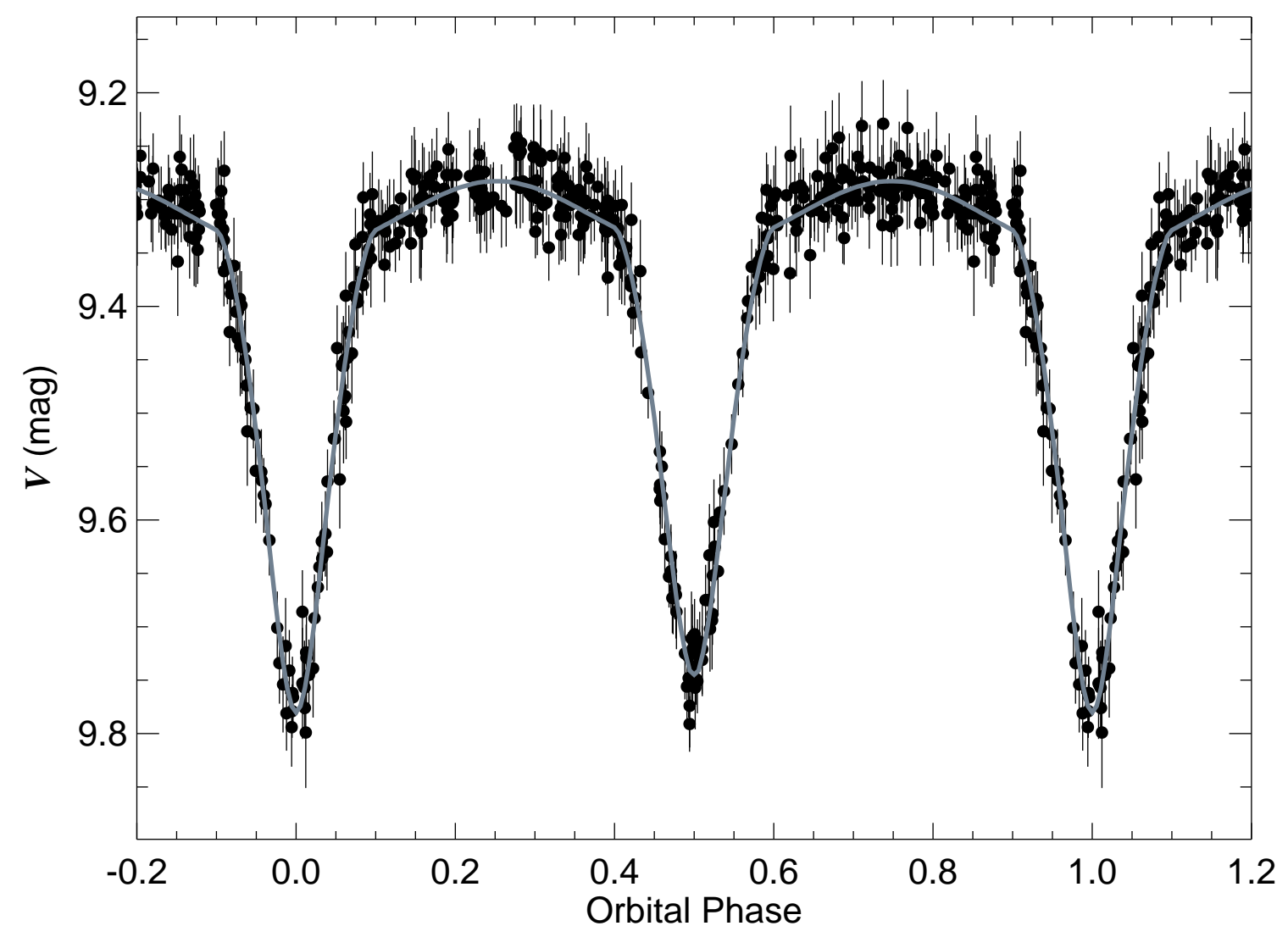

Fig. 5.- $V$-band light curve for HI Mon taken from the ASAS database (Pojmański 2002) and presented here in phase according to our best-fit solution. The model is the thick gray line and data are the filled dots with uncertainties represented by line segments. Phase zero for this plot corresponds to mid-eclipse of the primary star. 


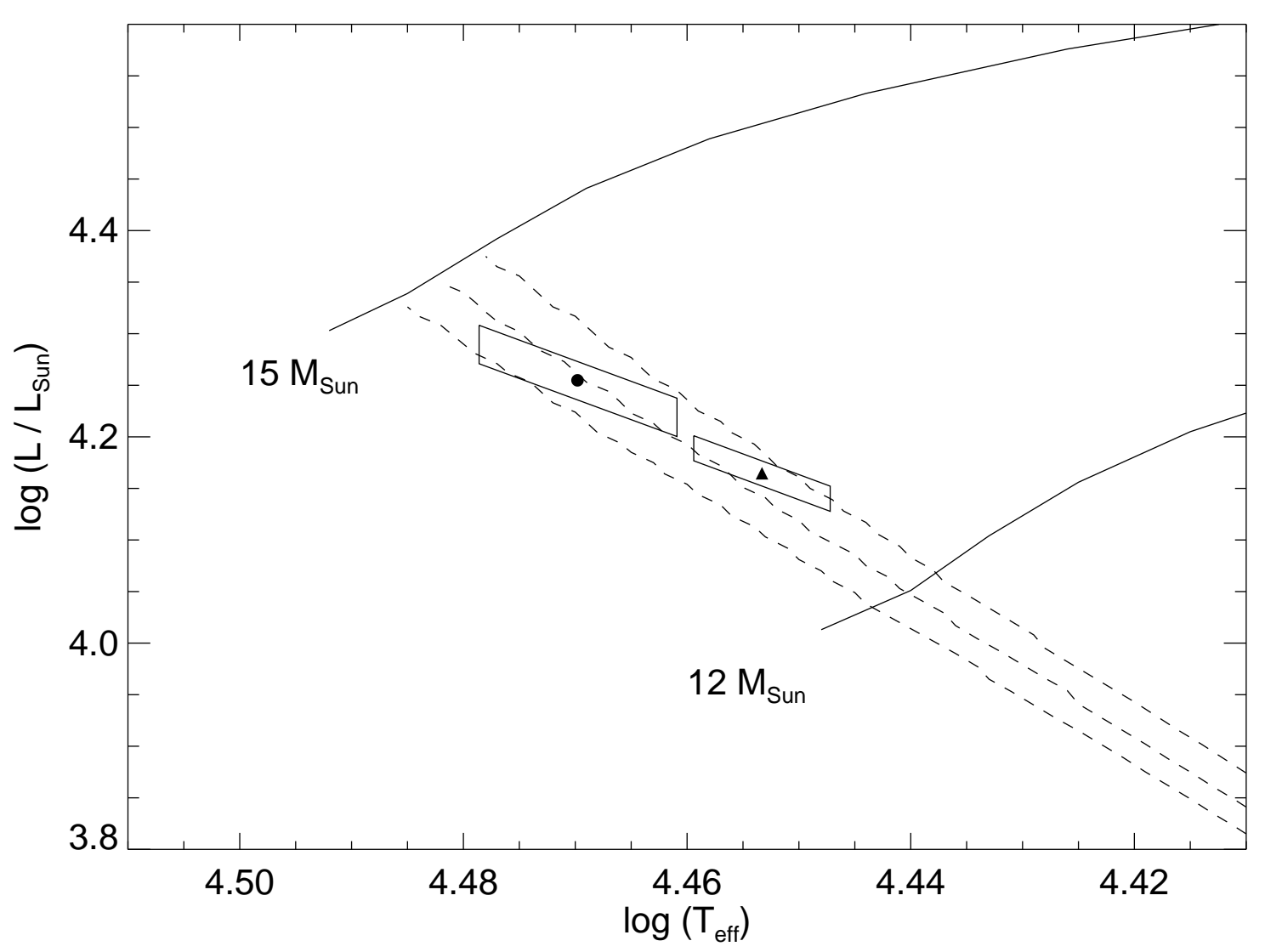

Fig. 6.- Theoretical H-R diagram showing the location of the primary star (filled circle) and secondary star (filled triangle) of HI Mon including uncertainty regions for each. Also plotted are the evolutionary tracks for stars of $12 M_{\odot}$ and $15 M_{\odot}$ from Schaller et al. (1992) and isochrones from Lejeune \& Schaerer (2001) for solar metallicity with ages of 1.5, 2.5, and 3.5 Myr going from lower left to upper right. The positions of the two components of HI Mon are consistent with an age of $\sim 2.5$ Myr. 


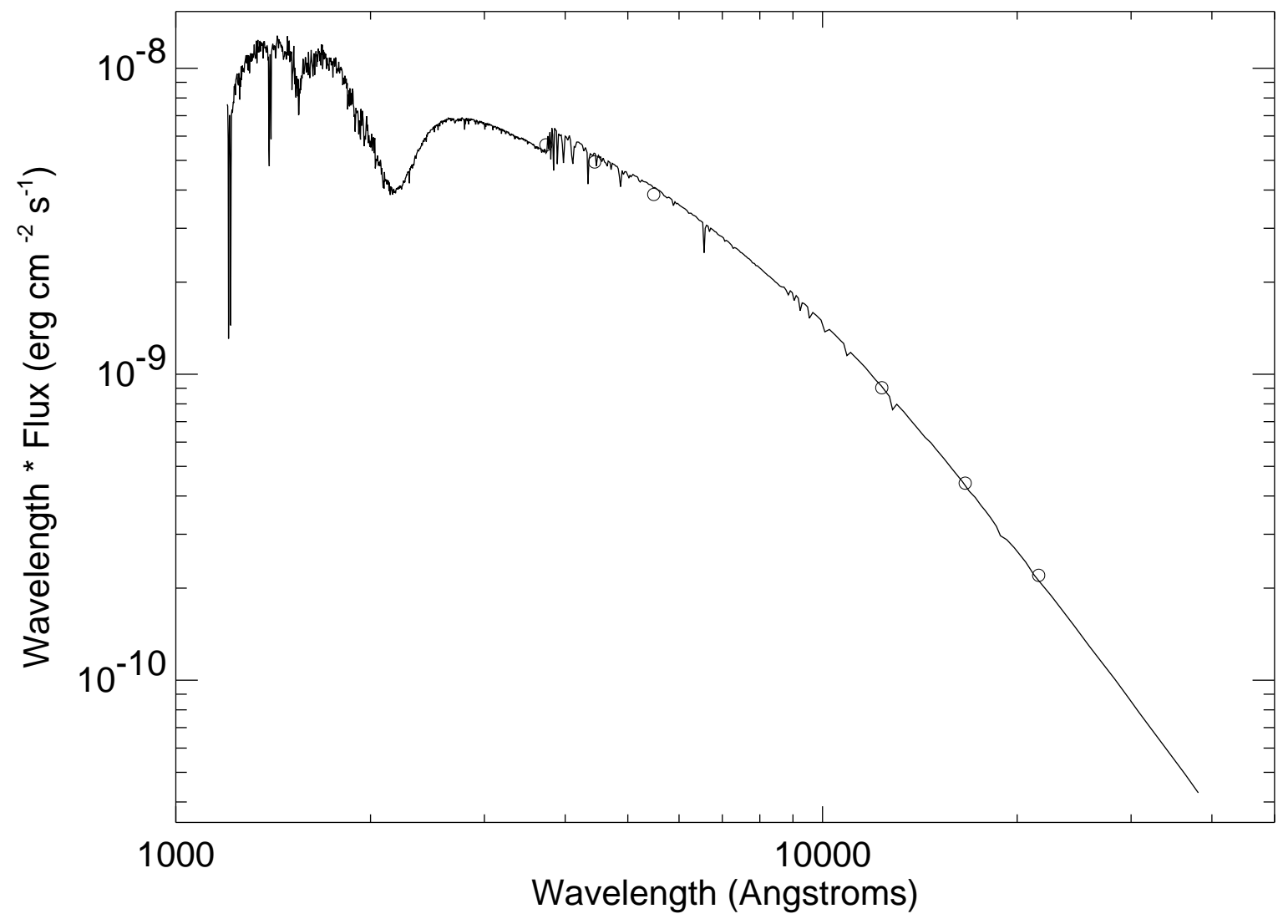

Fig. 7.- Spectral energy distribution and fit for the combined light of the HI Mon components (solid line) to Johnson $U, B, V, J, H, K_{S}$ photometry (open circles). 


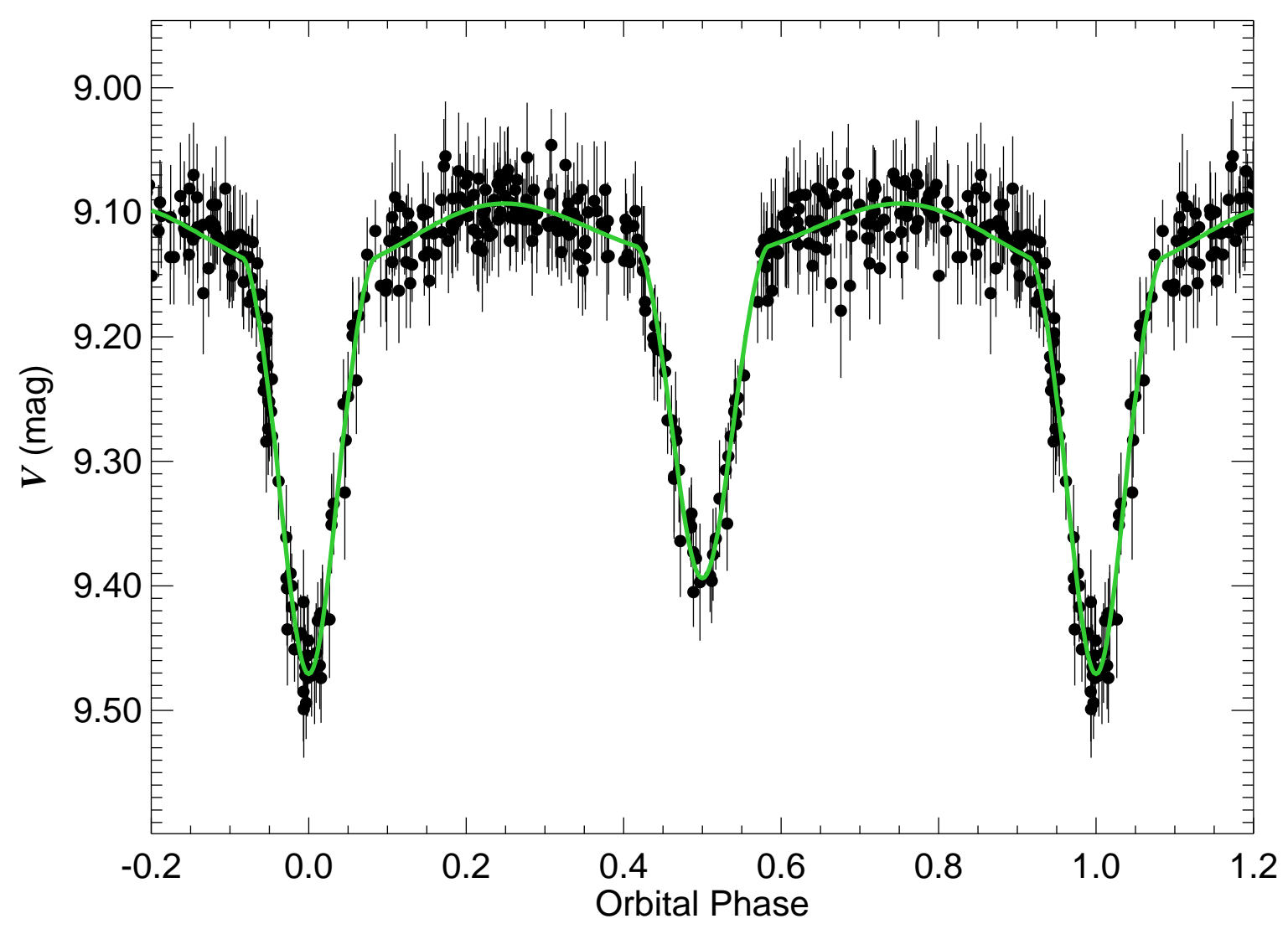

Fig. 8.- The ASAS $V$-band light curve for ASAS 053838+0901.2 (HD 37396). Filled circles with lines represent data with associated uncertainties. The best fit orbital solution listed in Table 2 is shown as a solid line passing through the data. 


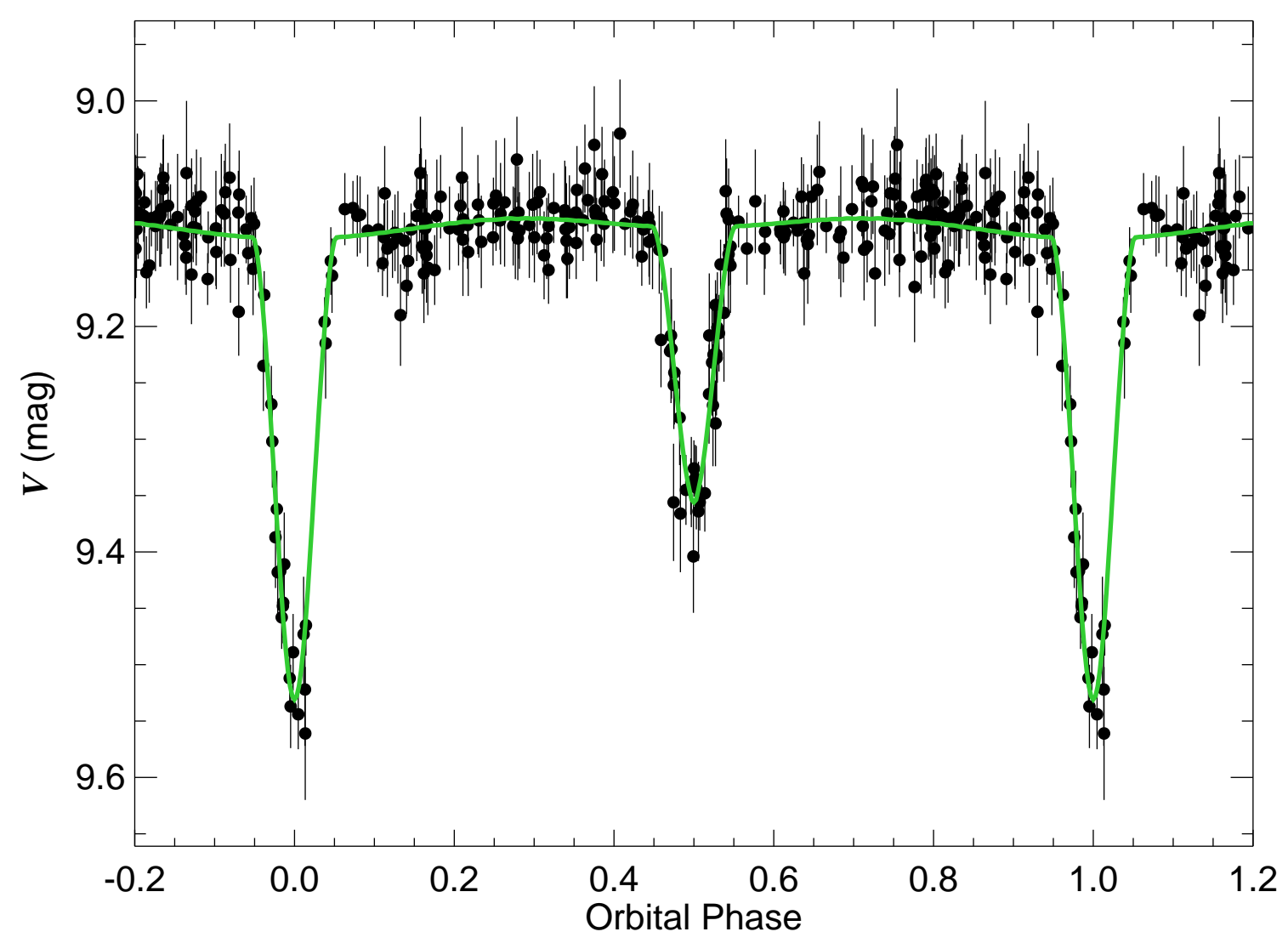

Fig. 9.- The ASAS $V$-band light curve for ASAS 054816+2046.1 (HD 247740). Filled circles with lines represent data with associated uncertainties. The best fit orbital solution listed in Table 2 is shown as a solid line passing through the data. 


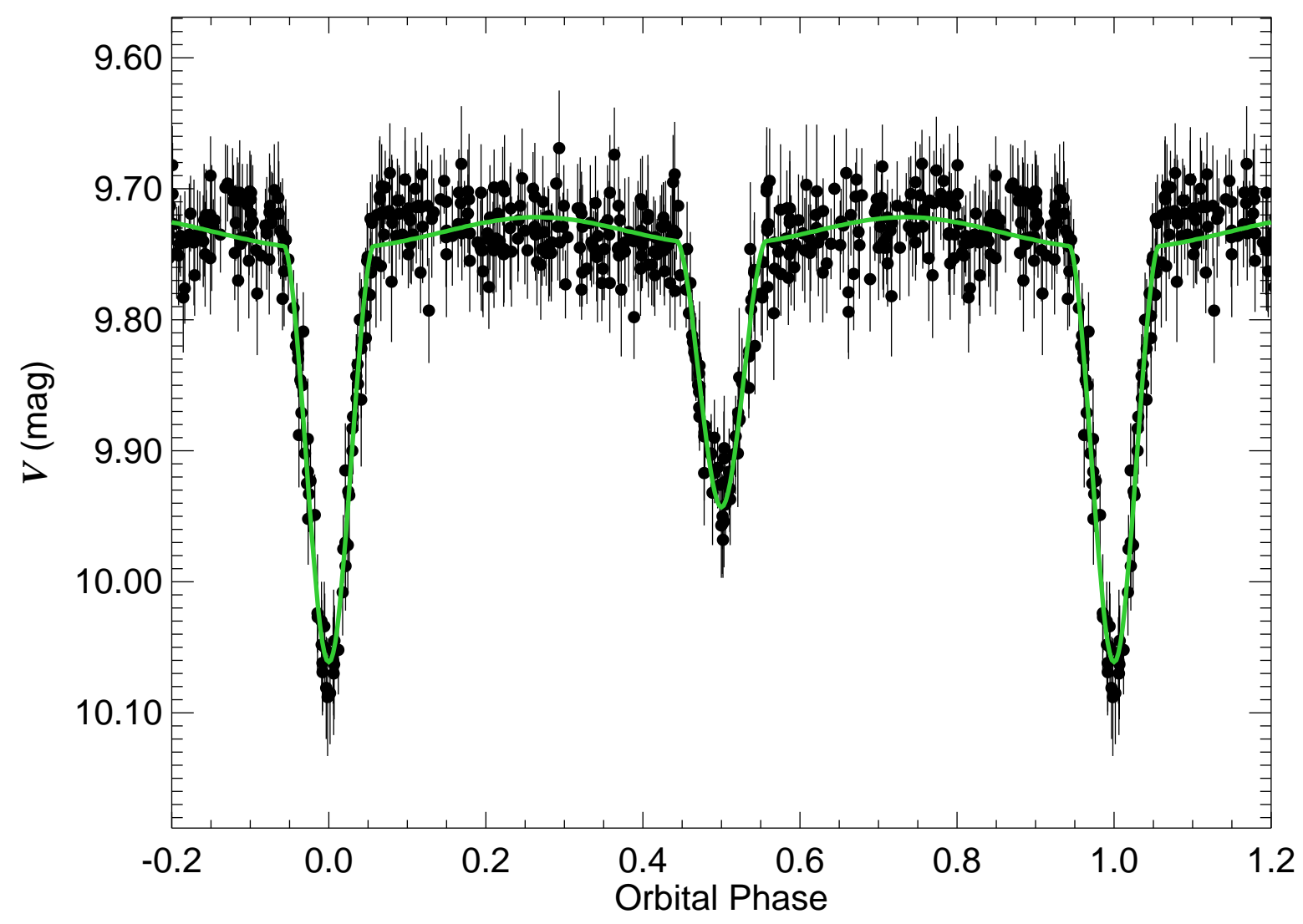

Fig. 10.- The ASAS $V$-band light curve for ASAS 060857+1128.9 (HD 252416). Filled circles with lines represent data with associated uncertainties. The best fit orbital solution listed in Table 2 is shown as a solid line passing through the data. 


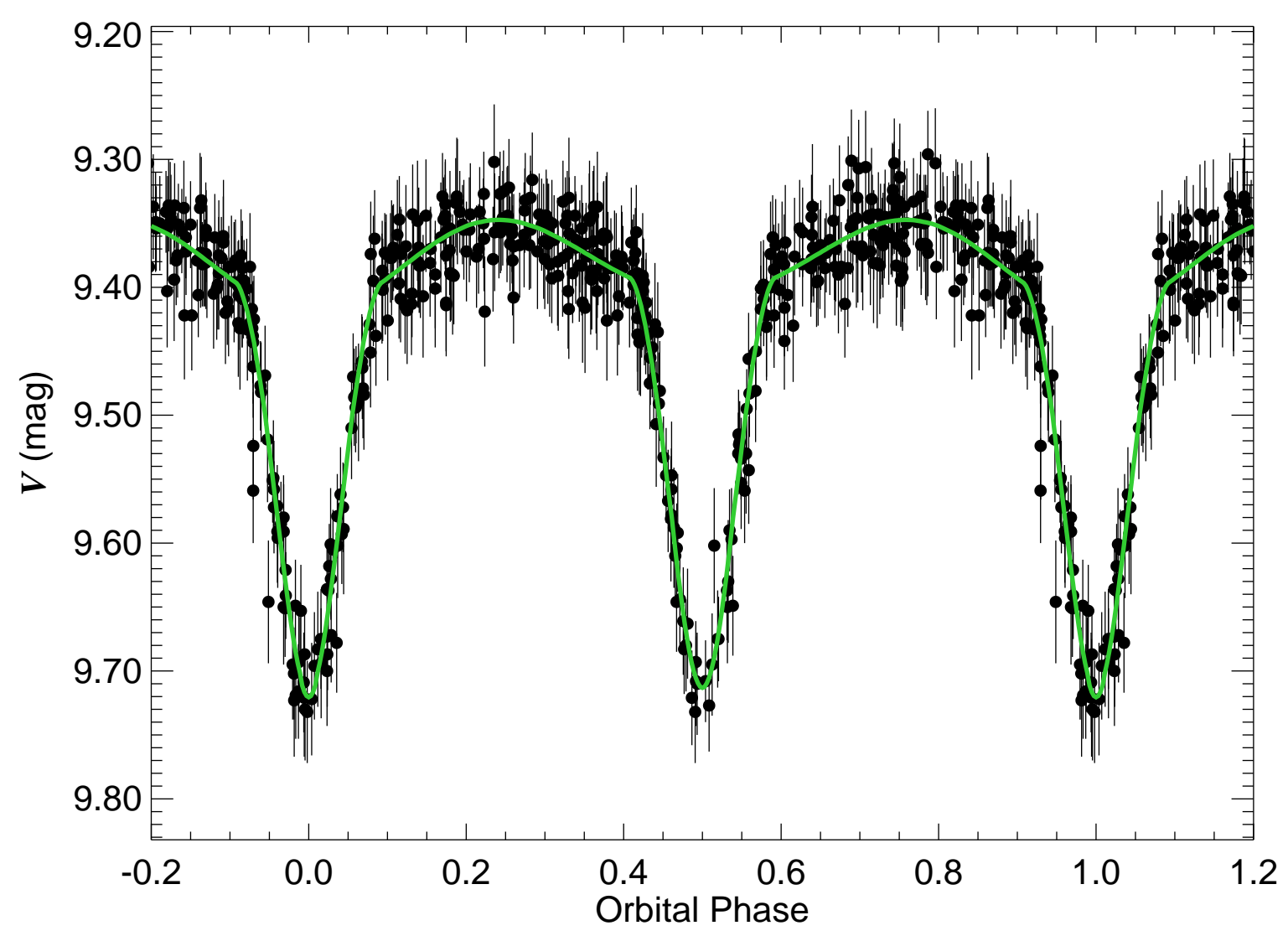

Fig. 11. - The ASAS $V$-band light curve for ASAS 060927-1501.7 (TYC 5933-142-1). Filled circles with lines represent data with associated uncertainties. The best fit orbital solution listed in Table 2 is shown as a solid line passing through the data. 


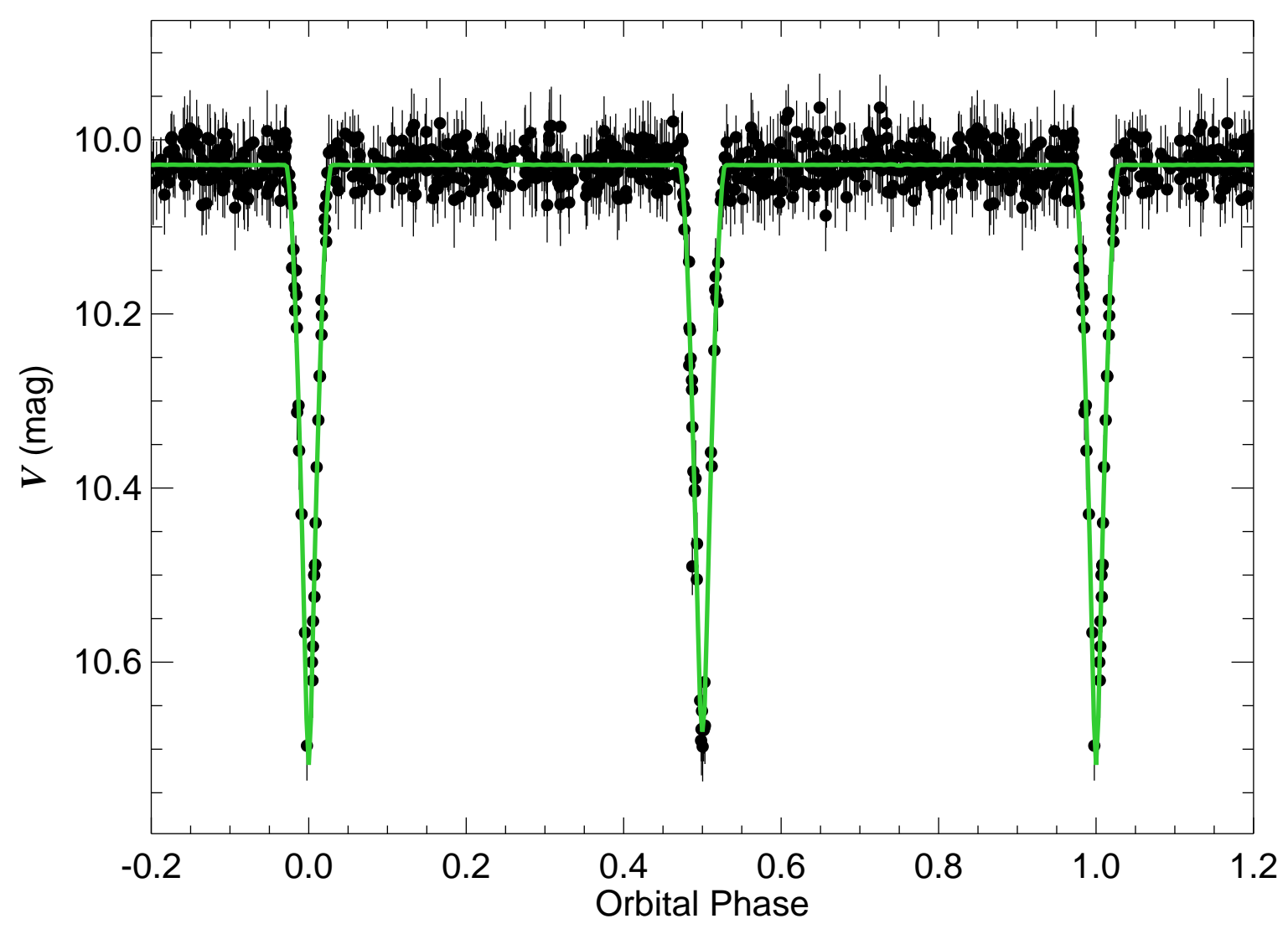

Fig. 12.- The ASAS $V$ band light curve for ASAS 062556-1254.5 (HD 45263). Filled circles with lines represent data with associated uncertainties. The best fit orbital solution listed in Table 2 is shown as a solid line passing through the data. 


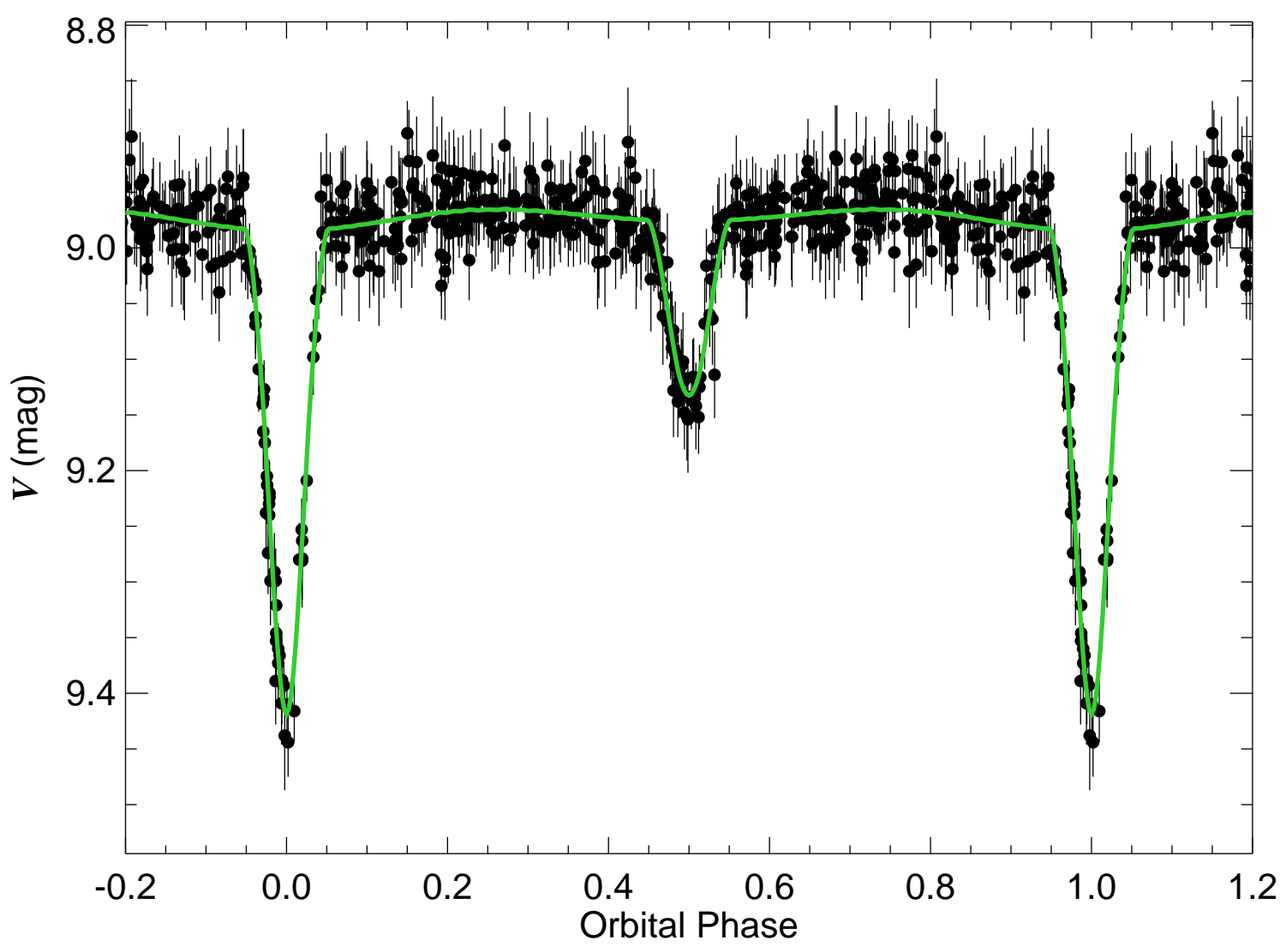

Fig. 13.- The ASAS $V$-band light curve for ASAS 063347-1410.5 (HD 46621). Filled circles with lines represent data with associated uncertainties. The best fit orbital solution listed in Table 2 is shown as a solid line passing through the data. 


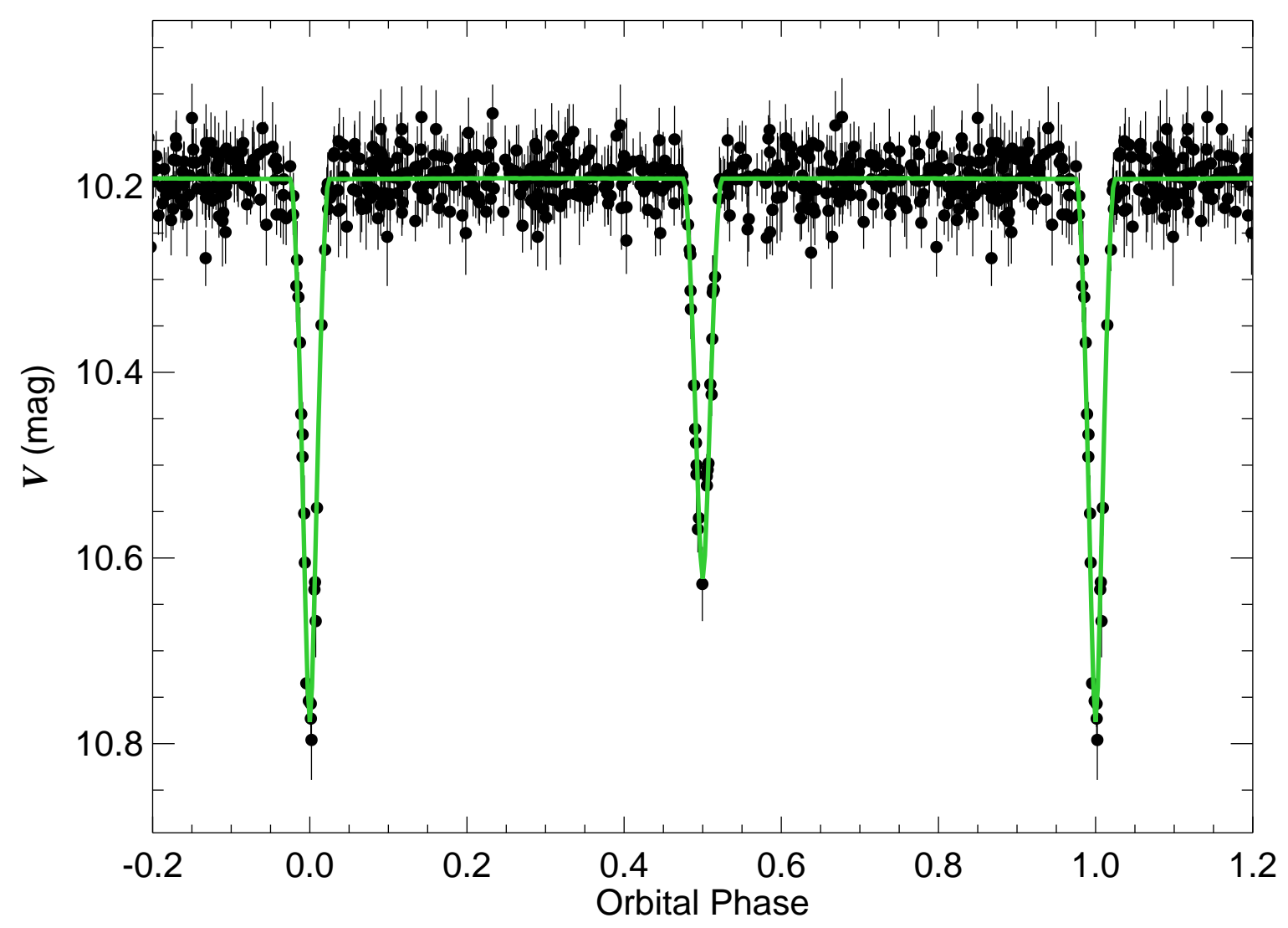

Fig. 14. - The ASAS $V$-band light curve for ASAS064010-1140.3 (HD 47845). Filled circles with lines represent data with associated uncertainties. The best fit orbital solution listed in Table 2 is shown as a solid line passing through the data. 


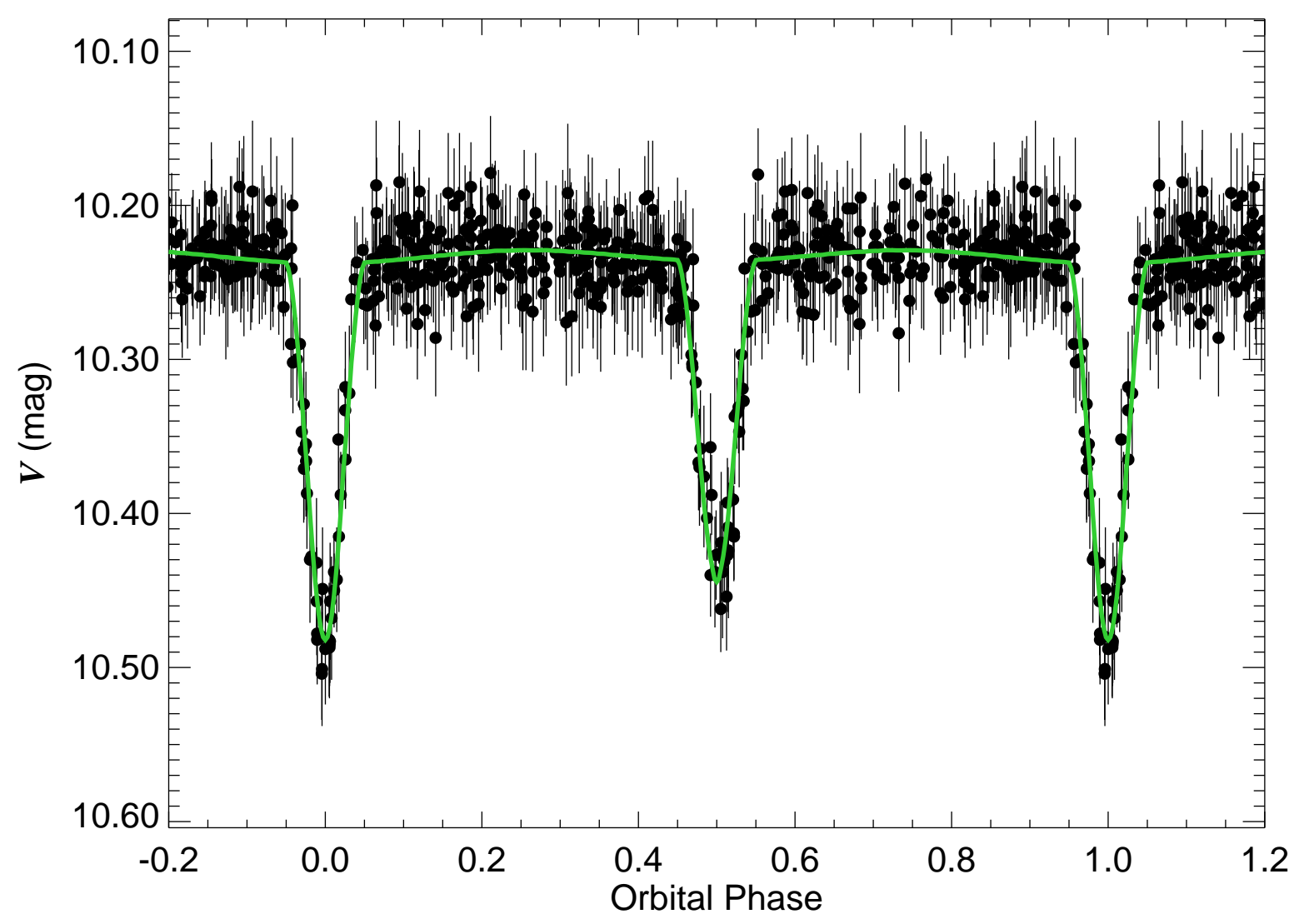

Fig. 15. - The ASAS $V$-band light curve for ASAS 064118-0551 (2MASS 06411762-0551065). Filled circles with lines represent data with associated uncertainties. The best fit orbital solution listed in Table 2 is shown as a solid line passing through the data. 


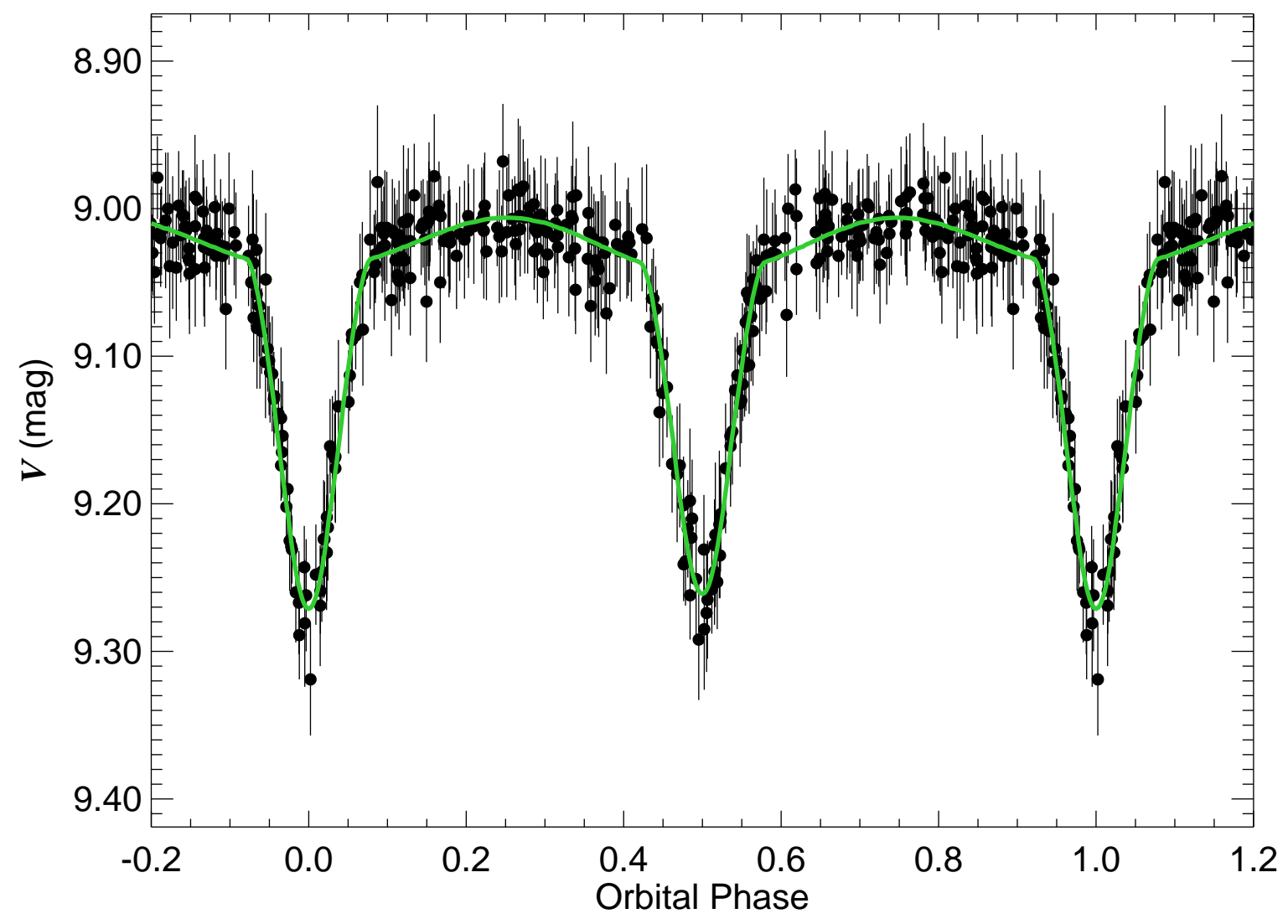

Fig. 16. - The ASAS $V$-band light curve for ASAS 064539+0219.4 (HD 48866). Filled circles with lines represent data with associated uncertainties. The best fit orbital solution listed in Table 2 is shown as a solid line passing through the data. 


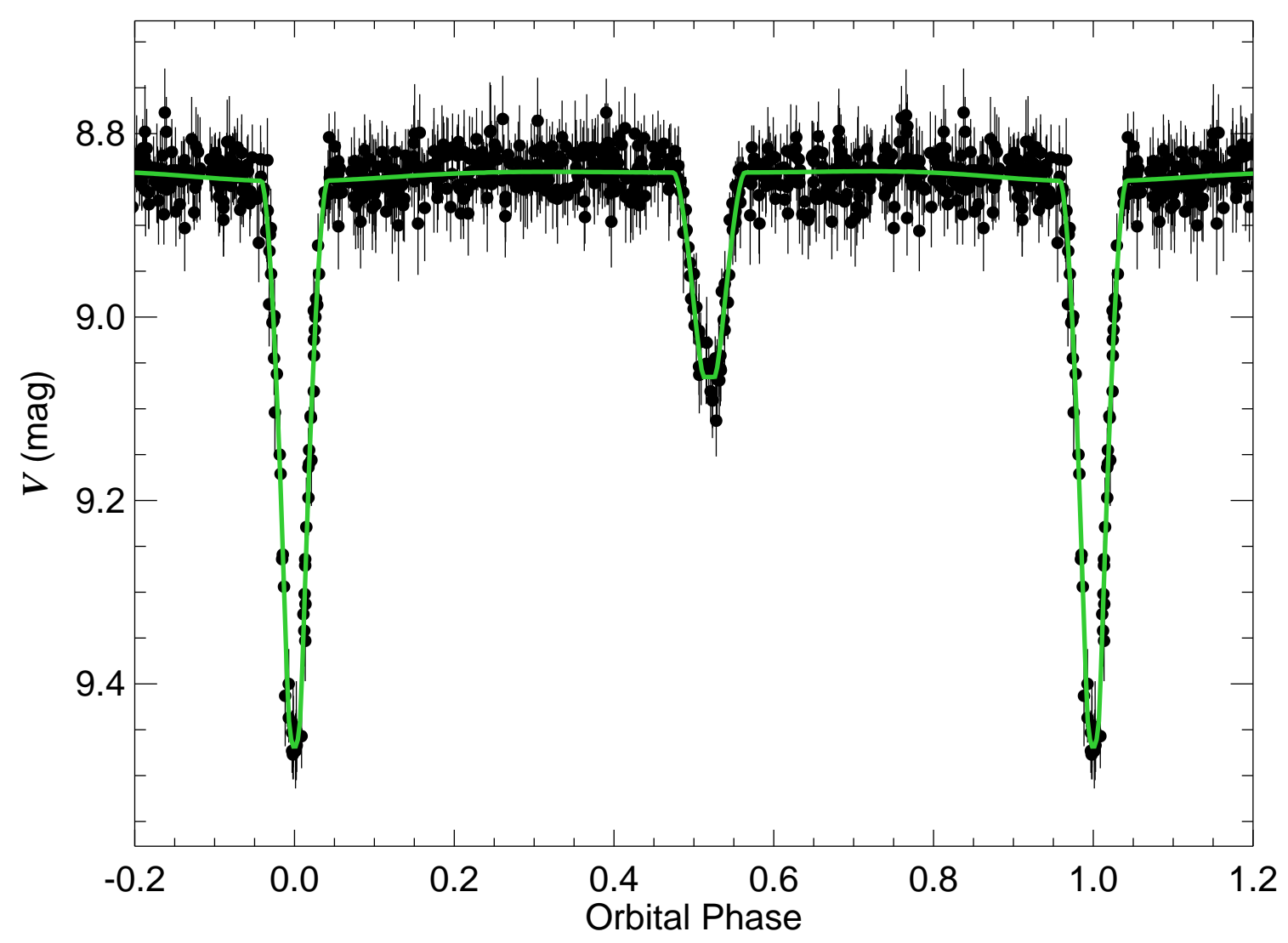

Fig. 17.- The ASAS $V$-band light curve for ASAS 064609-1923.8 (HD 49125). Filled circles with lines represent data with associated uncertainties. The best fit orbital solution listed in Table 2 is shown as a solid line passing through the data. 


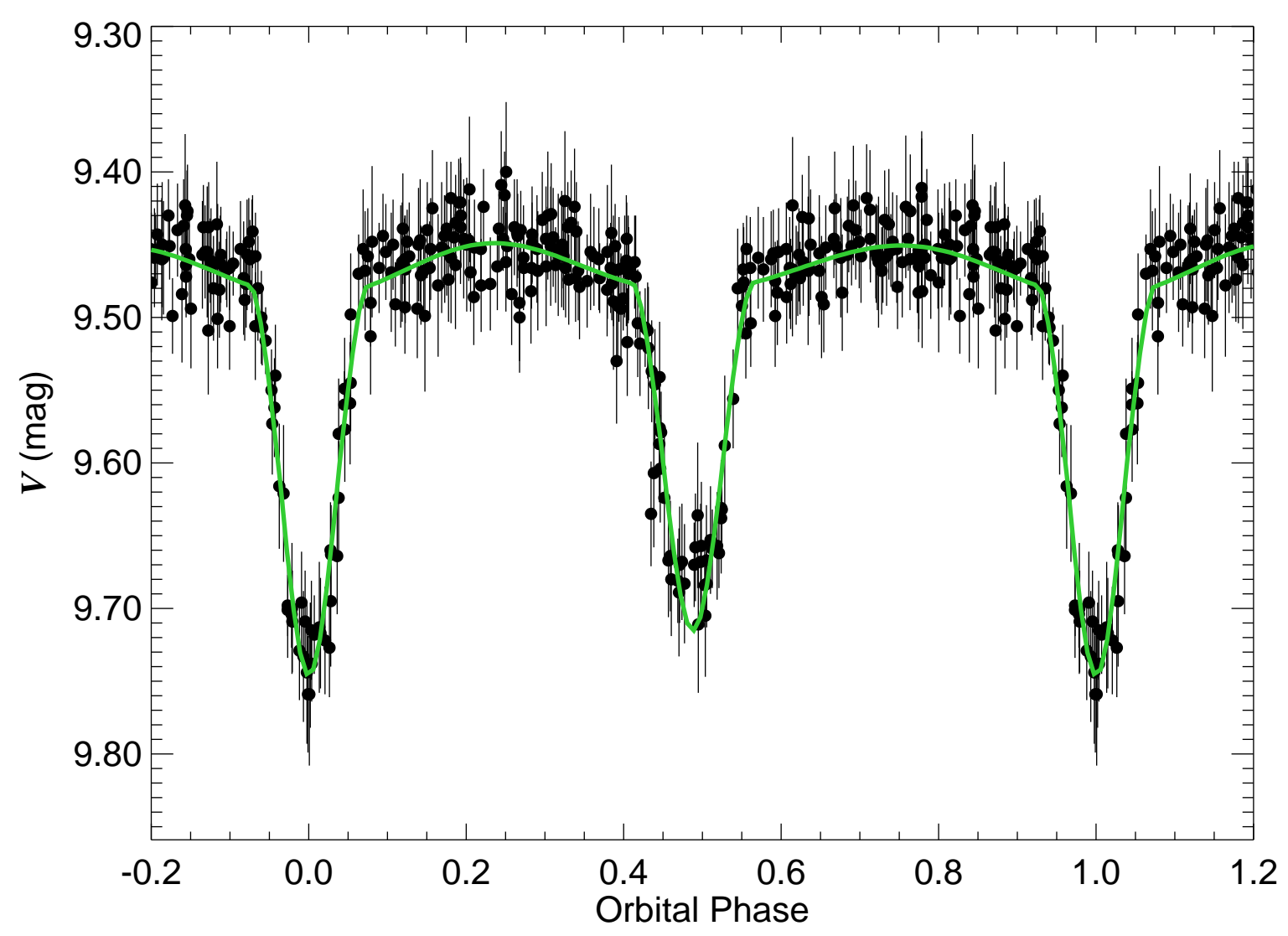

Fig. 18.- The ASAS $V$-band light curve for ASAS 064715+0225.6 (HD 289072). Filled circles with lines represent data with associated uncertainties. The best fit orbital solution listed in Table 2 is shown as a solid line passing through the data. 


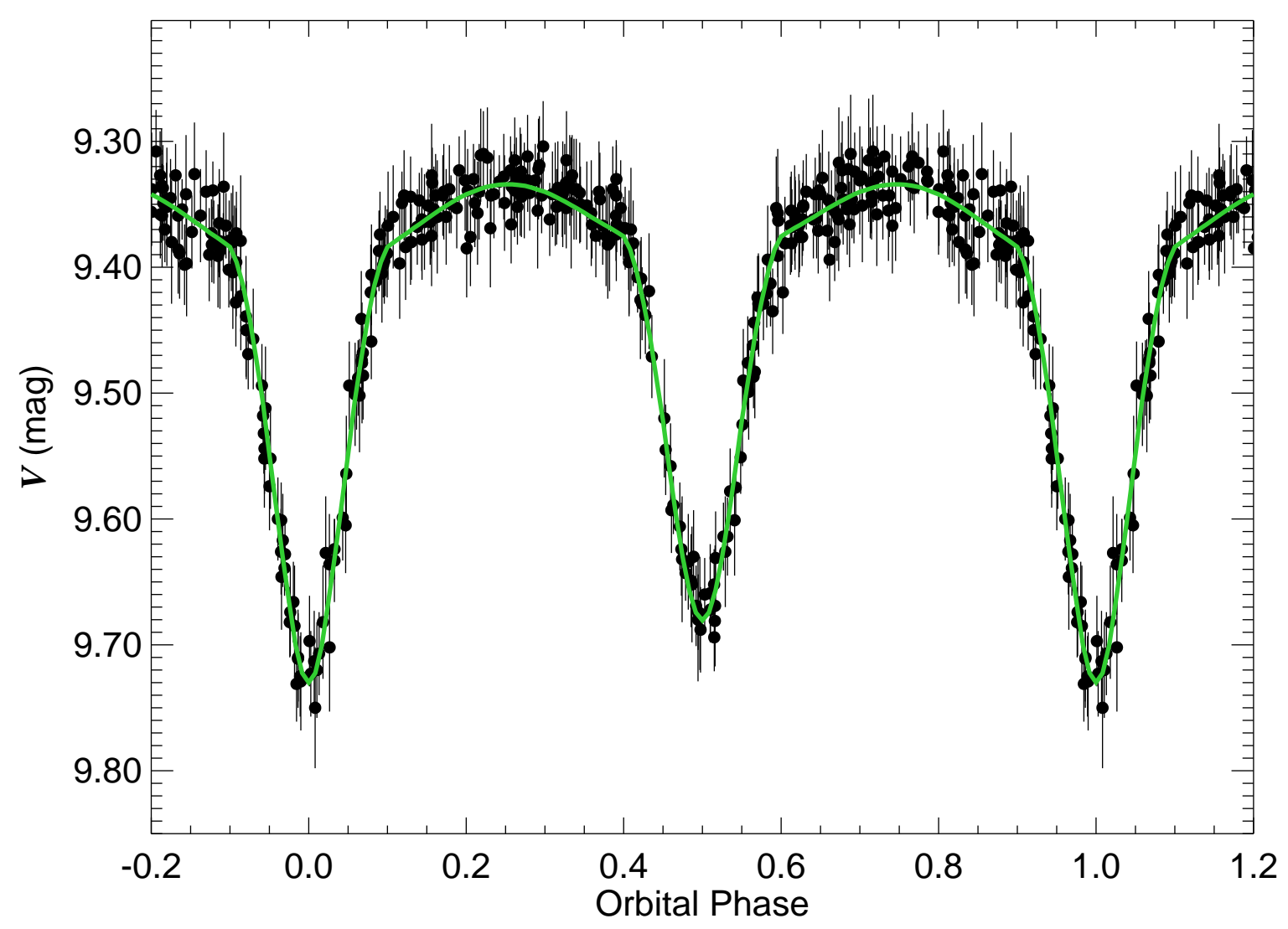

Fig. 19.- The ASAS $V$-band light curve for ASAS 064745+0122.3 (V448 Mon). Filled circles with lines represent data with associated uncertainties. The best fit orbital solution listed in Table 2 is shown as a solid line passing through the data. 


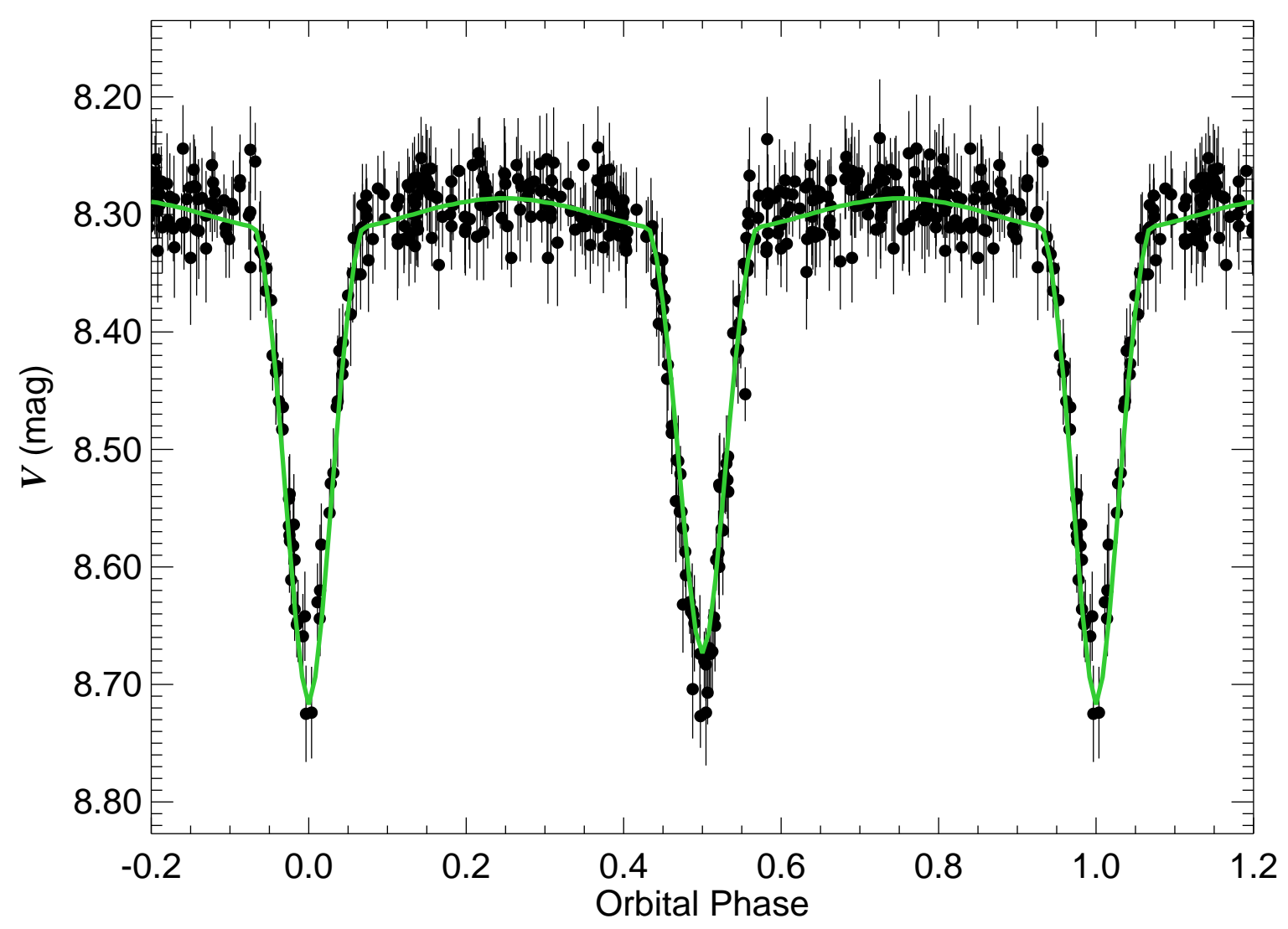

Fig. 20.- The ASAS $V$-band light curve for ASAS 065534-1013.2 (HD 51082). Filled circles with lines represent data with associated uncertainties. The best fit orbital solution listed in Table 2 is shown as a solid line passing through the data. 


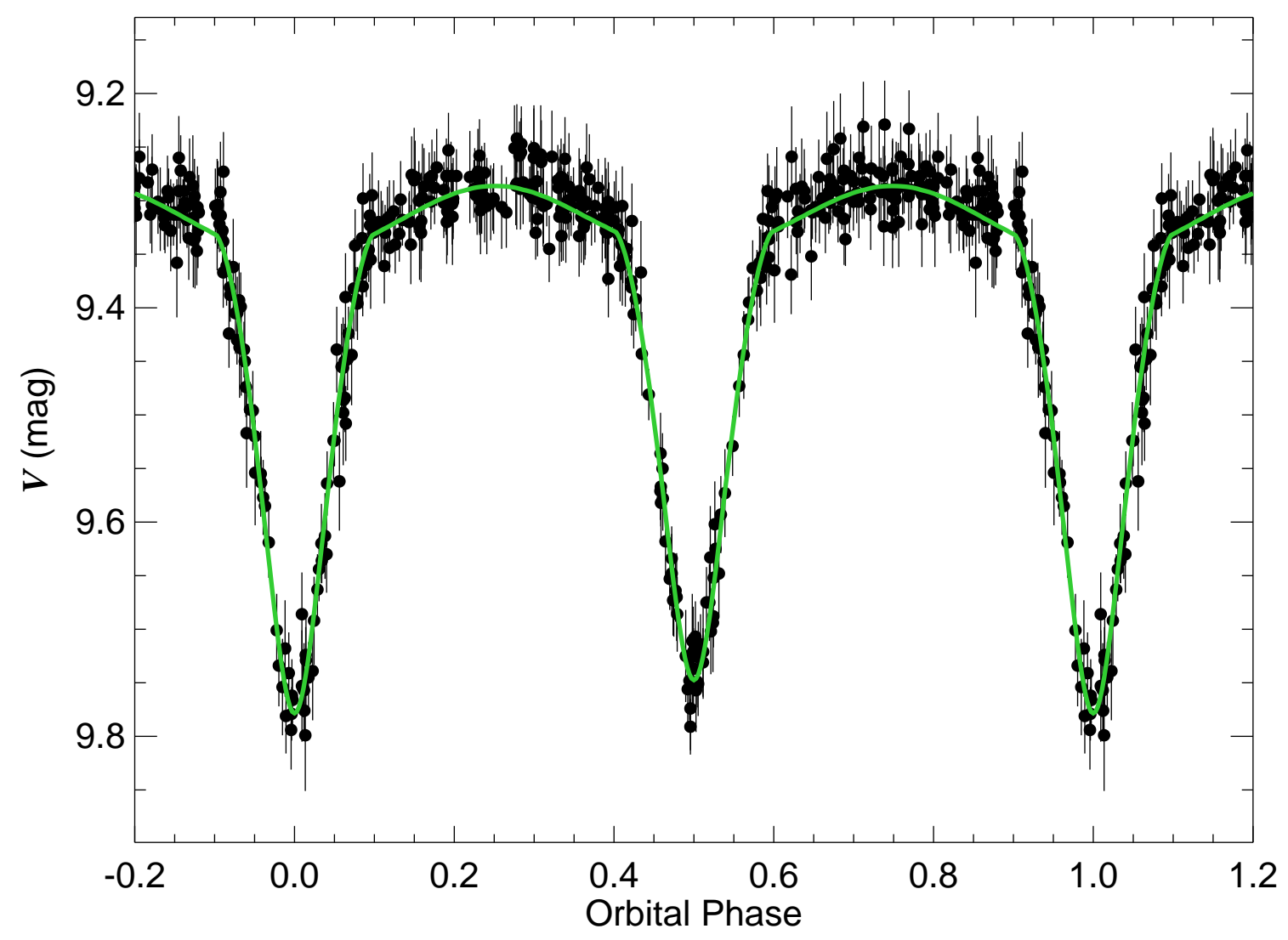

Fig. 21.- The ASAS $V$-band light curve for ASAS 065549-0402.6 (HI Mon). Filled circles with lines represent data with associated uncertainties. The best fit orbital solution listed in Table 2 is shown as a solid line passing through the data. 


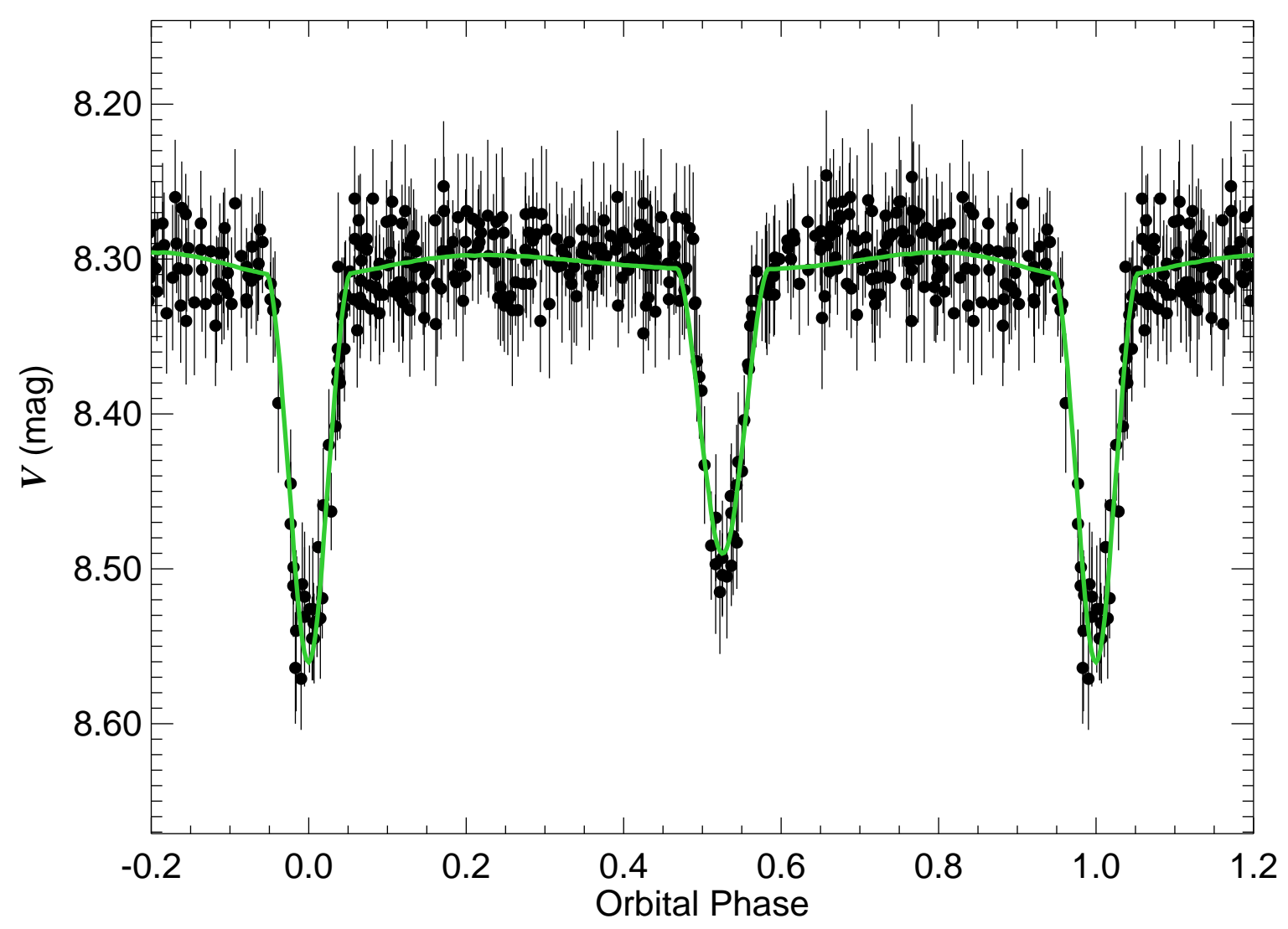

Fig. 22.- The ASAS $V$-band light curve for ASAS 070105-0358.2 (HD 52433). Filled circles with lines represent data with associated uncertainties. The best fit orbital solution listed in Table 2 is shown as a solid line passing through the data. 


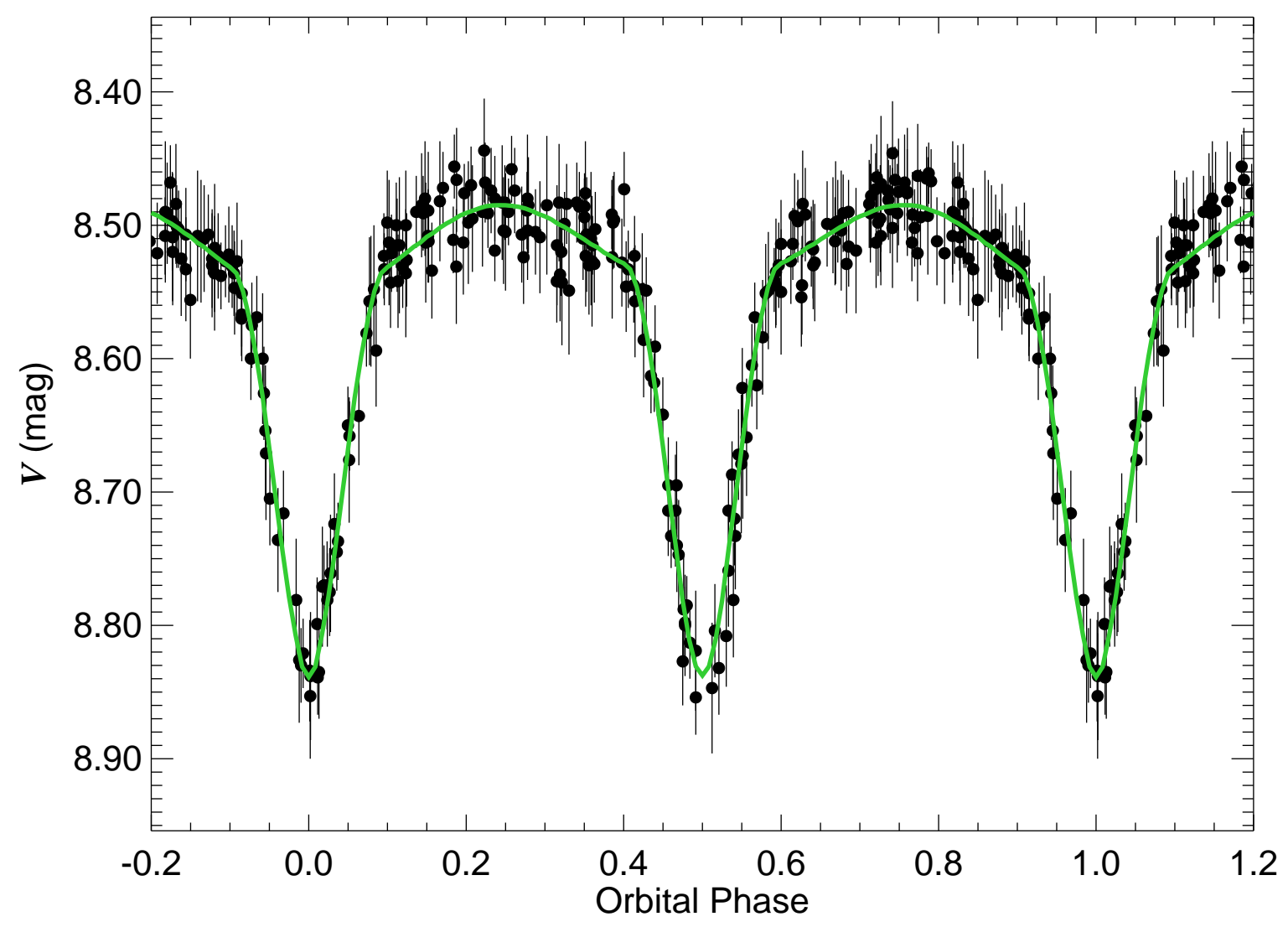

Fig. 23. - The ASAS $V$-band light curve for ASAS 070238+1347.0 (HD 52637). Filled circles with lines represent data with associated uncertainties. The best fit orbital solution listed in Table 2 is shown as a solid line passing through the data. 


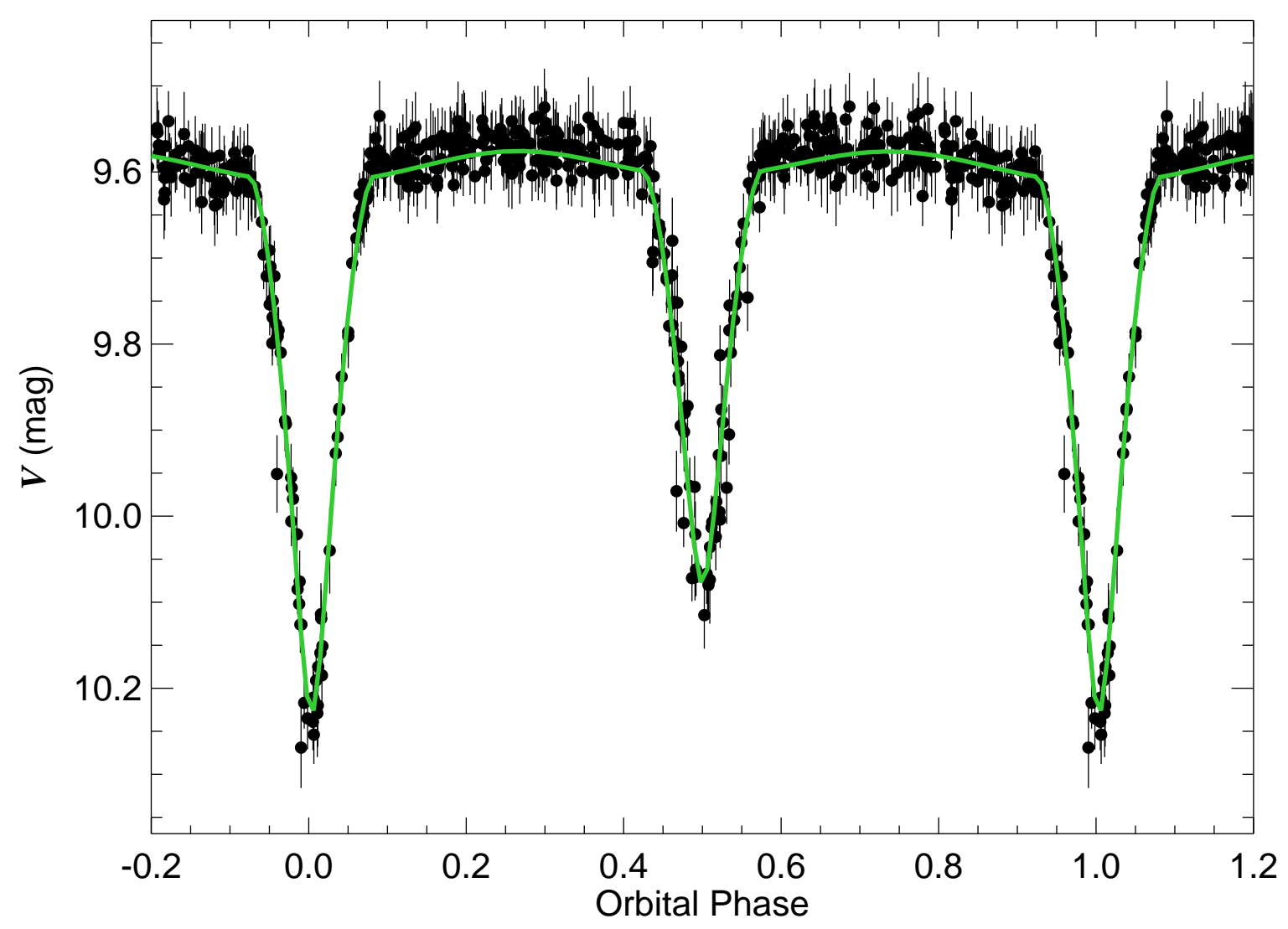

Fig. 24.- The ASAS $V$-band light curve for ASAS 070636-0437.4 (AO Mon). Filled circles with lines represent data with associated uncertainties. The best fit orbital solution listed in Table 2 is shown as a solid line passing through the data. 


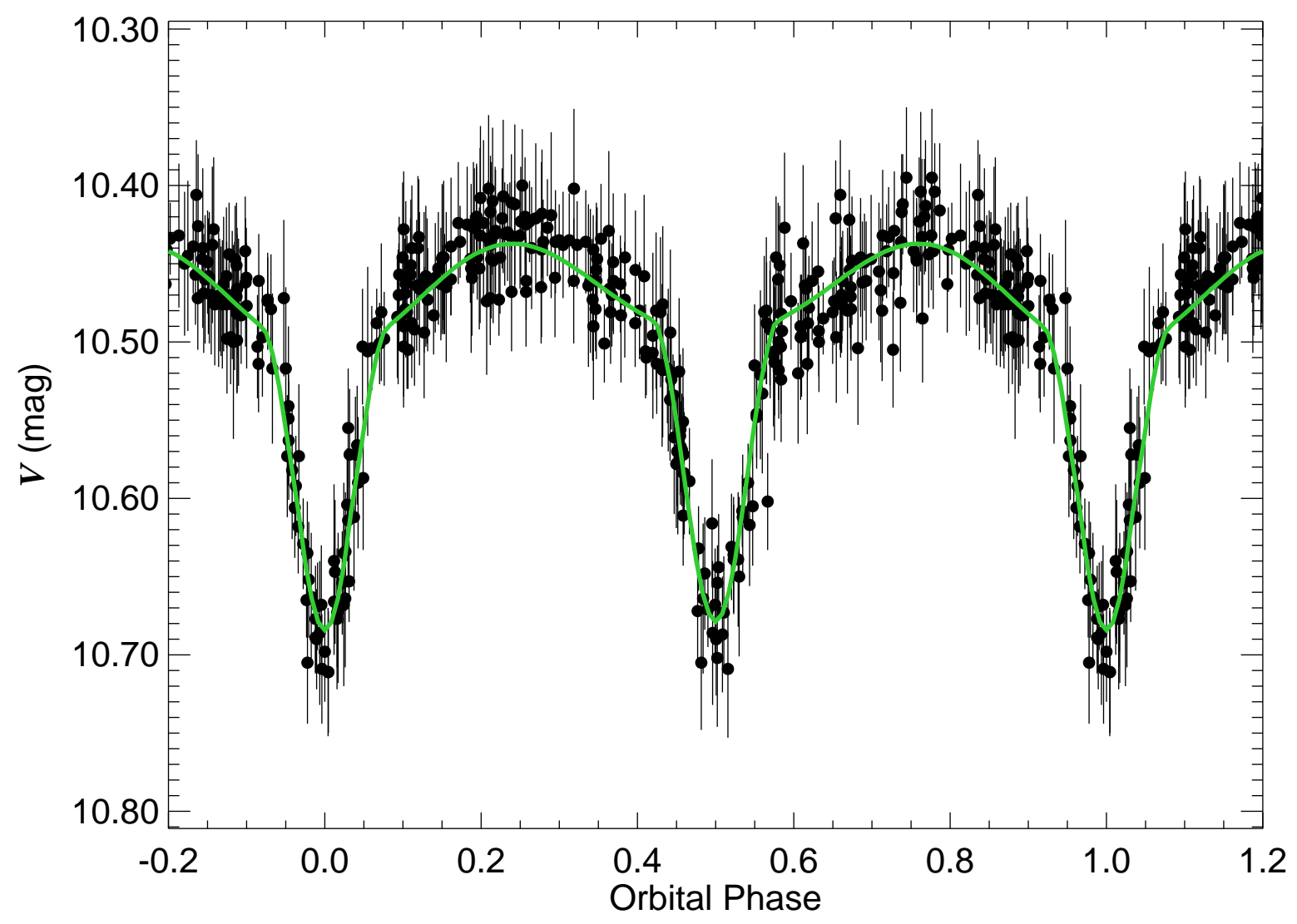

Fig. 25. - The ASAS $V$-band light curve for ASAS 070943+2341.7 (BD +23 1621). Filled circles with lines represent data with associated uncertainties. The best fit orbital solution listed in Table 2 is shown as a solid line passing through the data. 


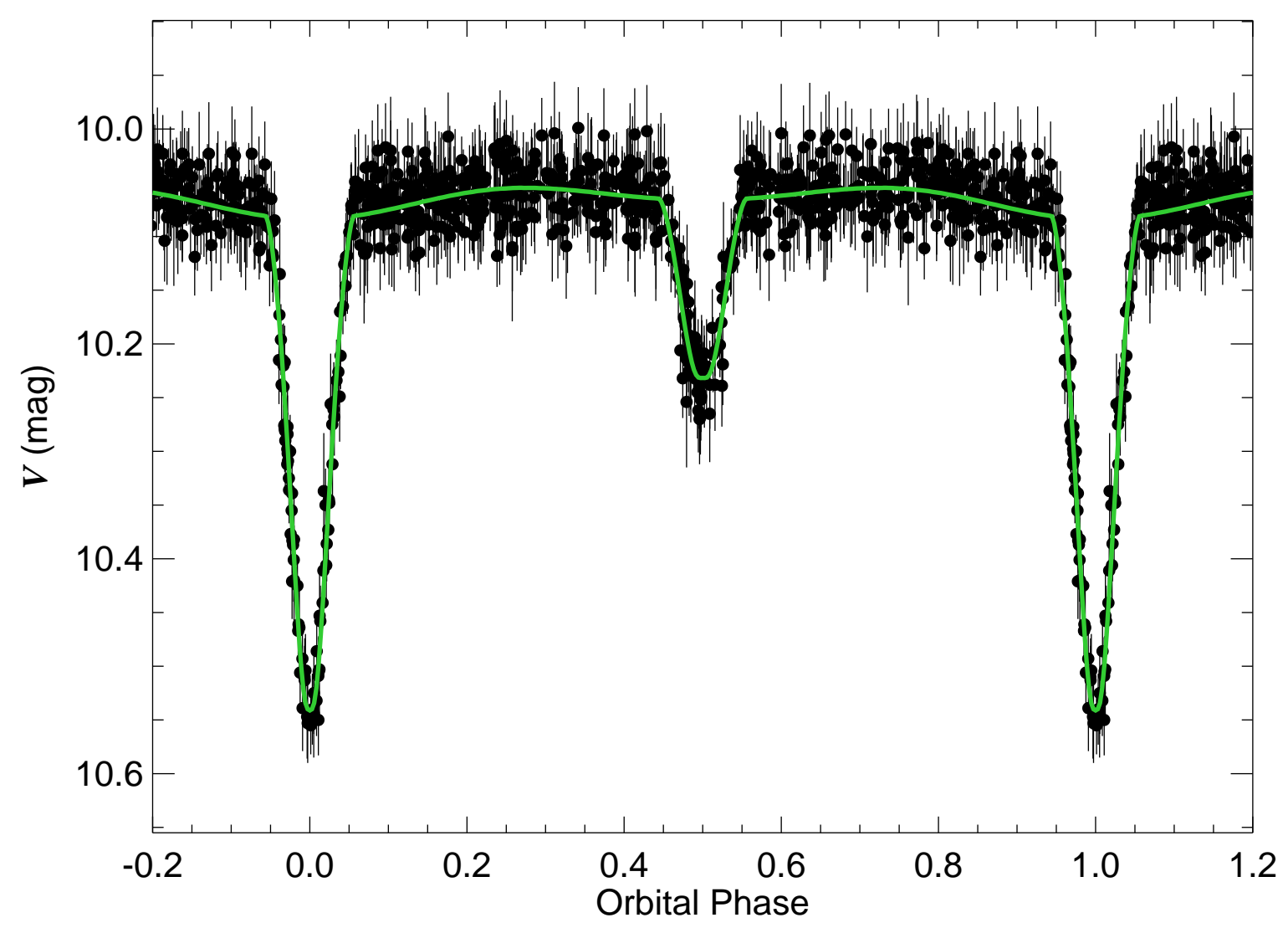

Fig. 26. - The ASAS $V$-band light curve for ASAS 070946-2005.5 (NSV 3433). Filled circles with lines represent data with associated uncertainties. The best fit orbital solution listed in Table 2 is shown as a solid line passing through the data. 


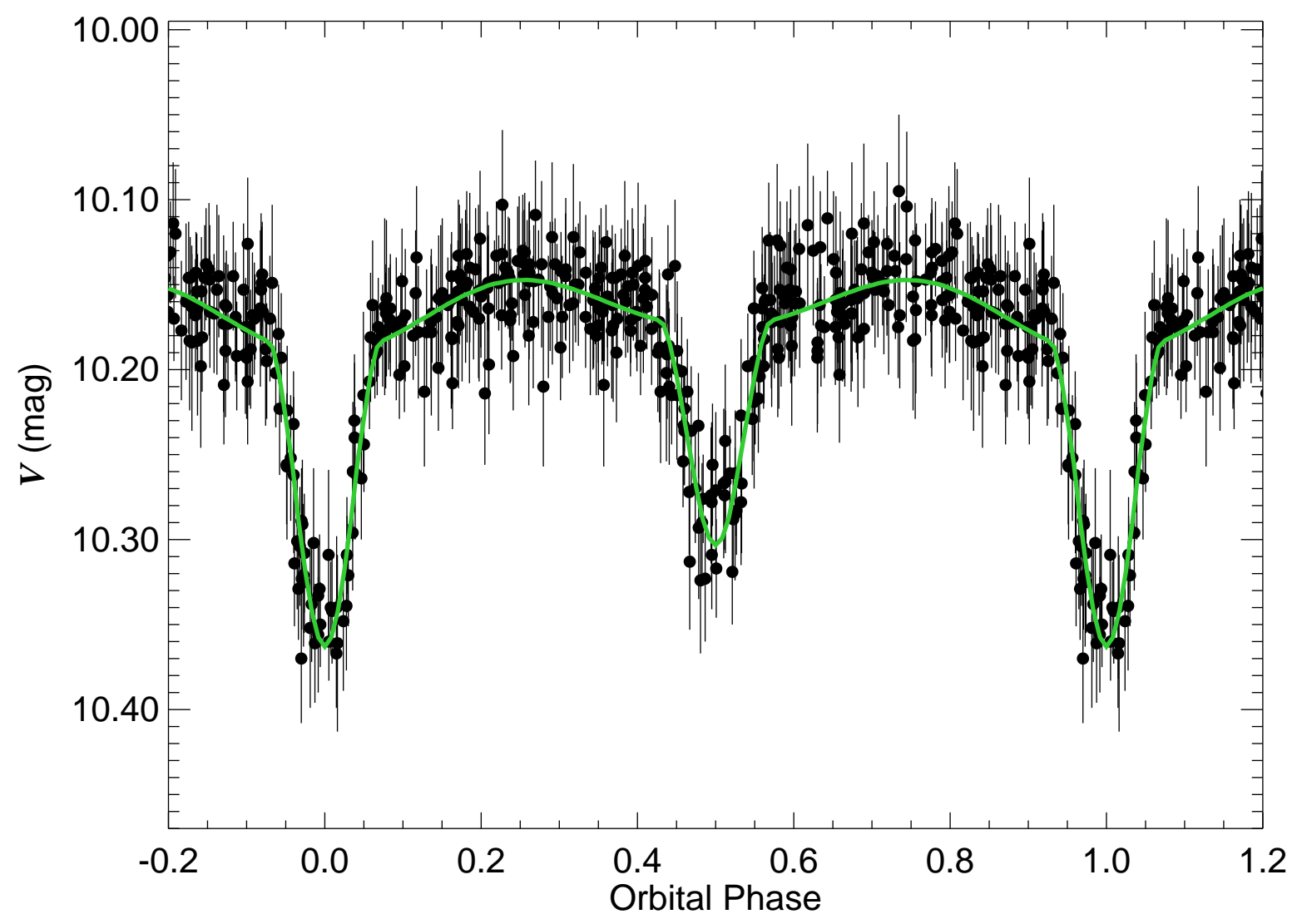

Fig. 27.- The ASAS $V$-band light curve for ASAS 071010-0035.1 (HD 54780). Filled circles with lines represent data with associated uncertainties. The best fit orbital solution listed in Table 2 is shown as a solid line passing through the data. 


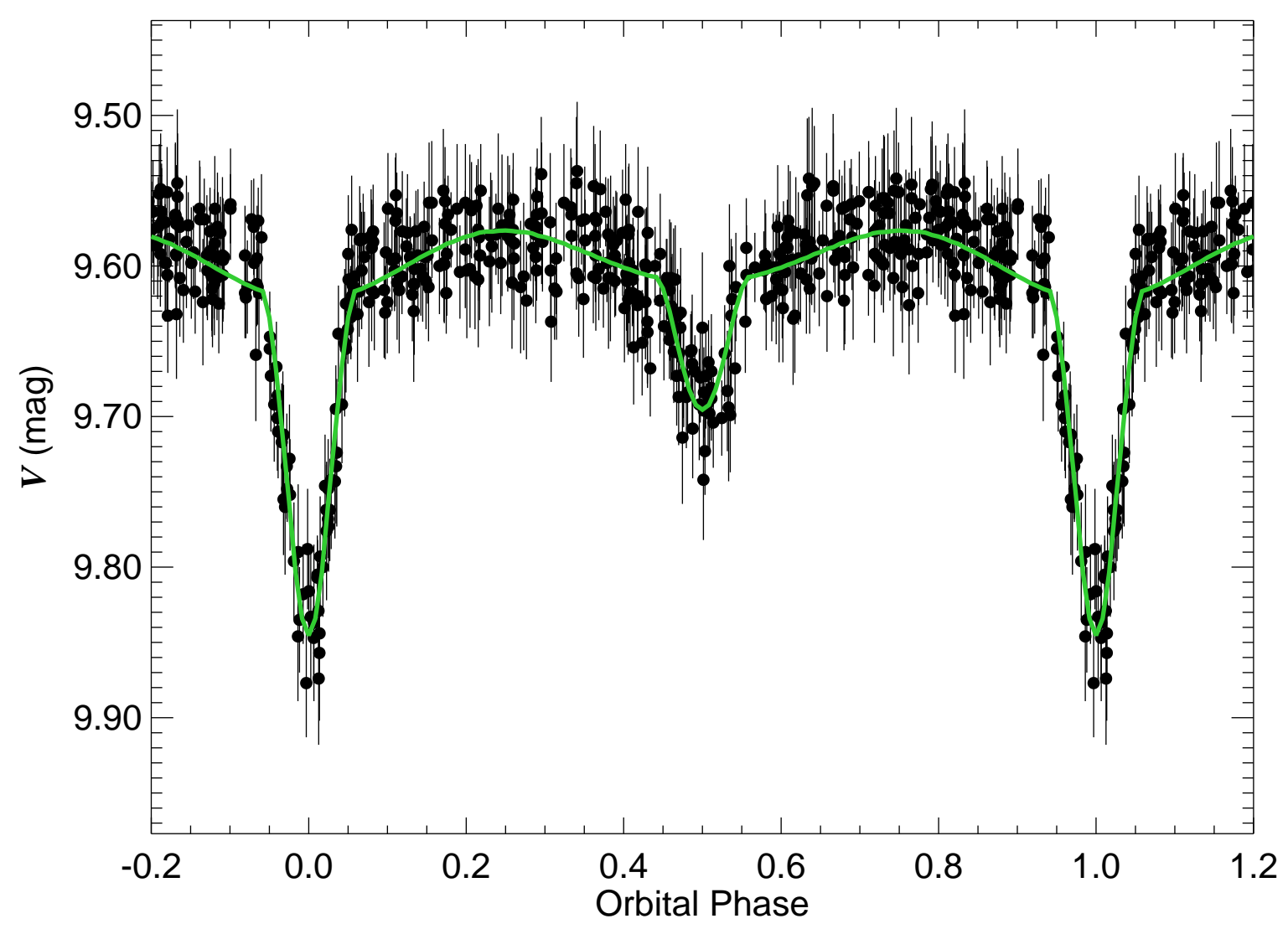

Fig. 28. - The ASAS $V$-band light curve for ASAS 071203-0139.1 (HD 55236). Filled circles with lines represent data with associated uncertainties. The best fit orbital solution listed in Table 2 is shown as a solid line passing through the data. 


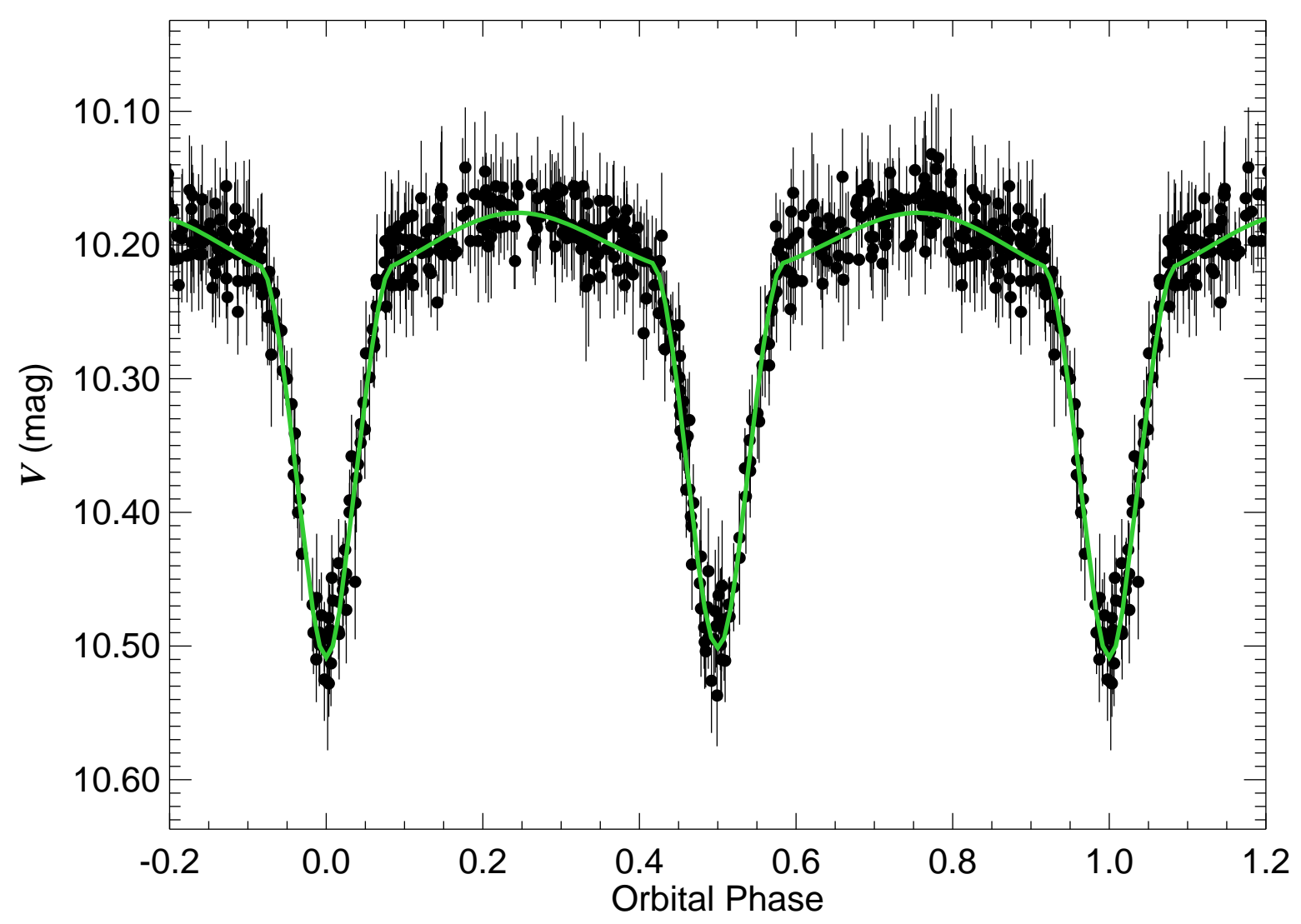

Fig. 29.- The ASAS $V$-band light curve for ASAS 071702-1034.9 (HD 56544). Filled circles with lines represent data with associated uncertainties. The best fit orbital solution listed in Table 2 is shown as a solid line passing through the data. 


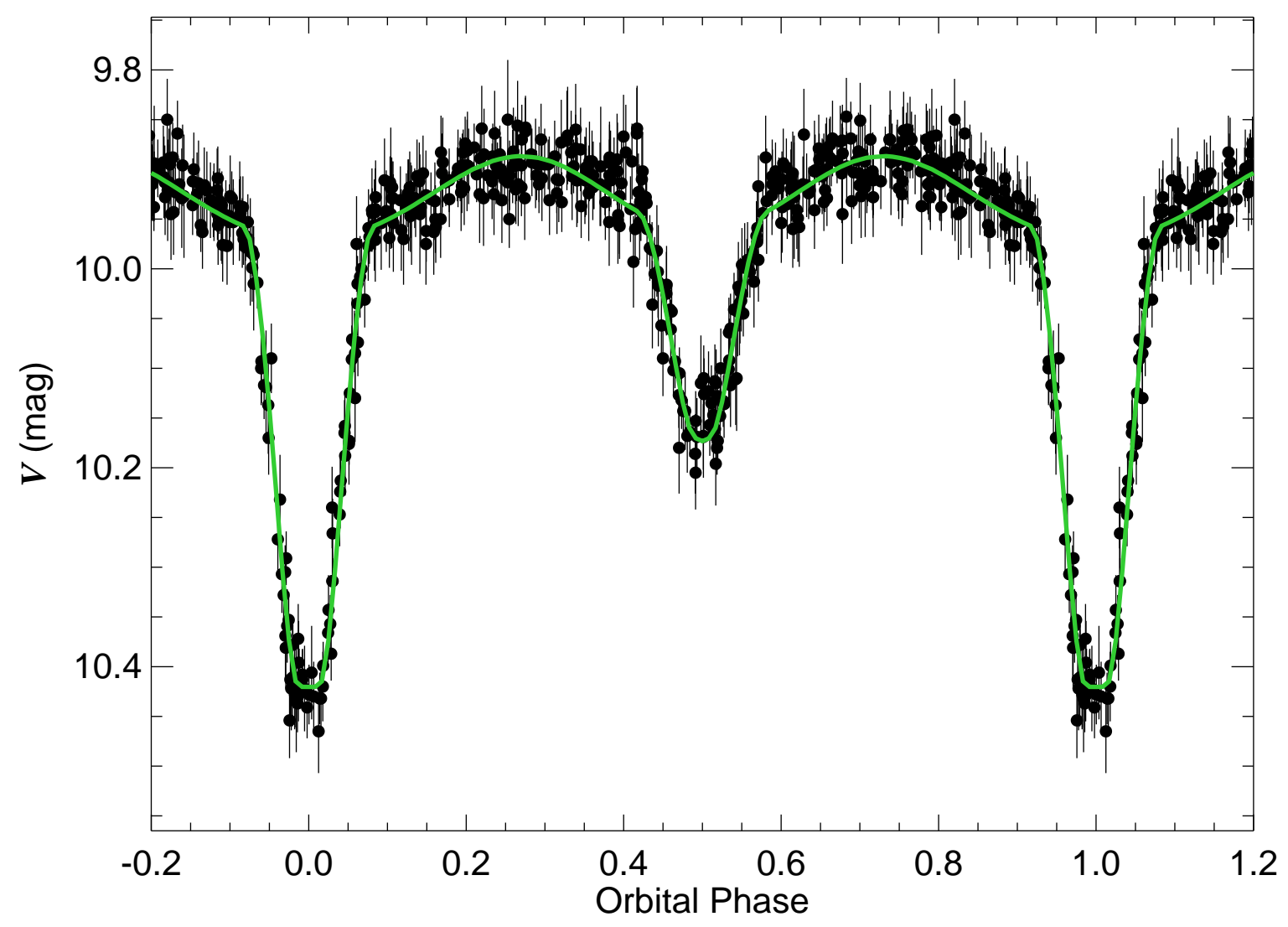

Fig. 30.- The ASAS $V$-band light curve for ASAS 072201-2552.6 (CX CMa). Filled circles with lines represent data with associated uncertainties. The best fit orbital solution listed in Table 2 is shown as a solid line passing through the data. 


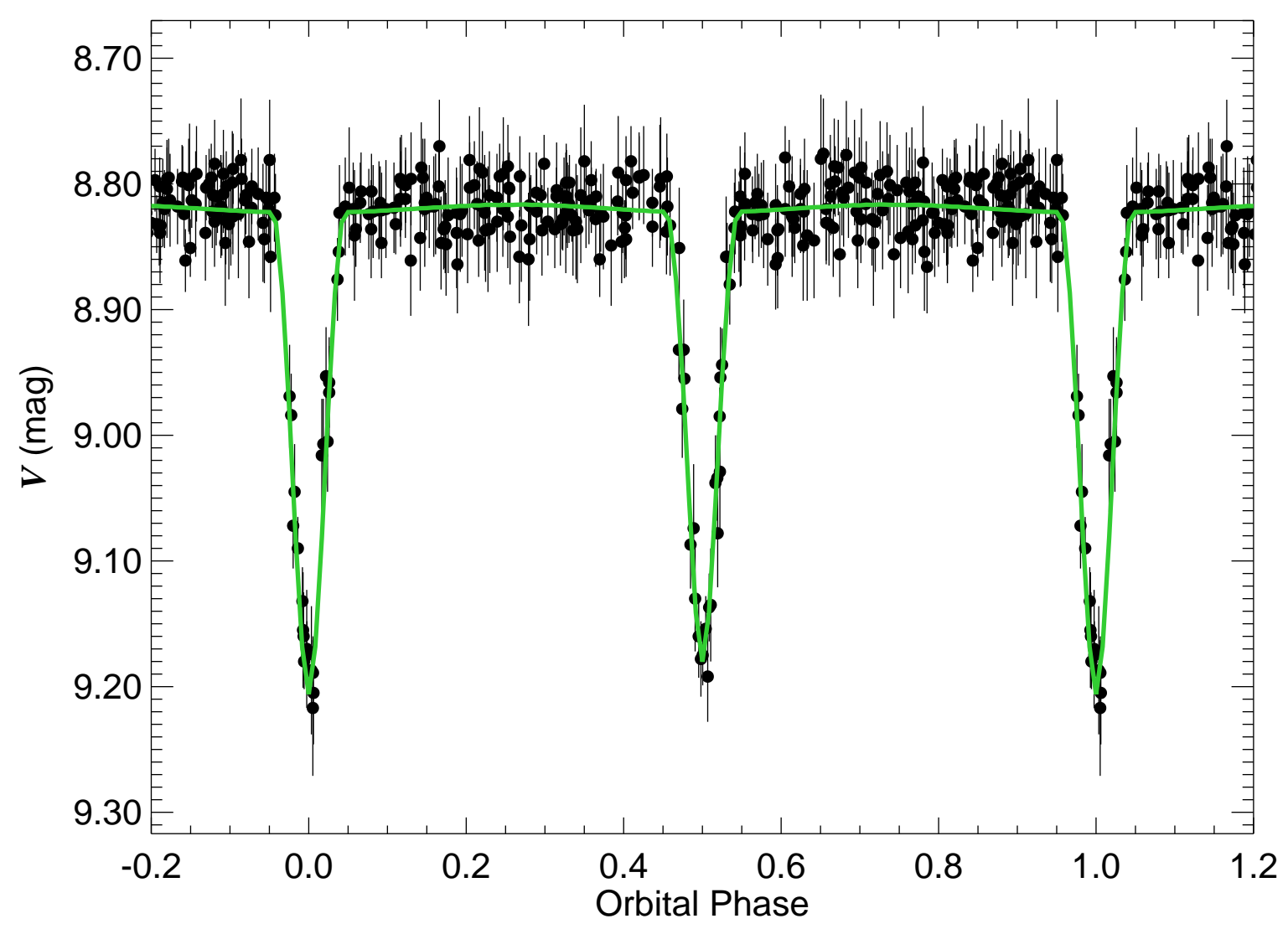

Fig. 31. - The ASAS $V$-band light curve for ASAS 073053+0513.7 (HD 59607). Filled circles with lines represent data with associated uncertainties. The best fit orbital solution listed in Table 2 is shown as a solid line passing through the data. 


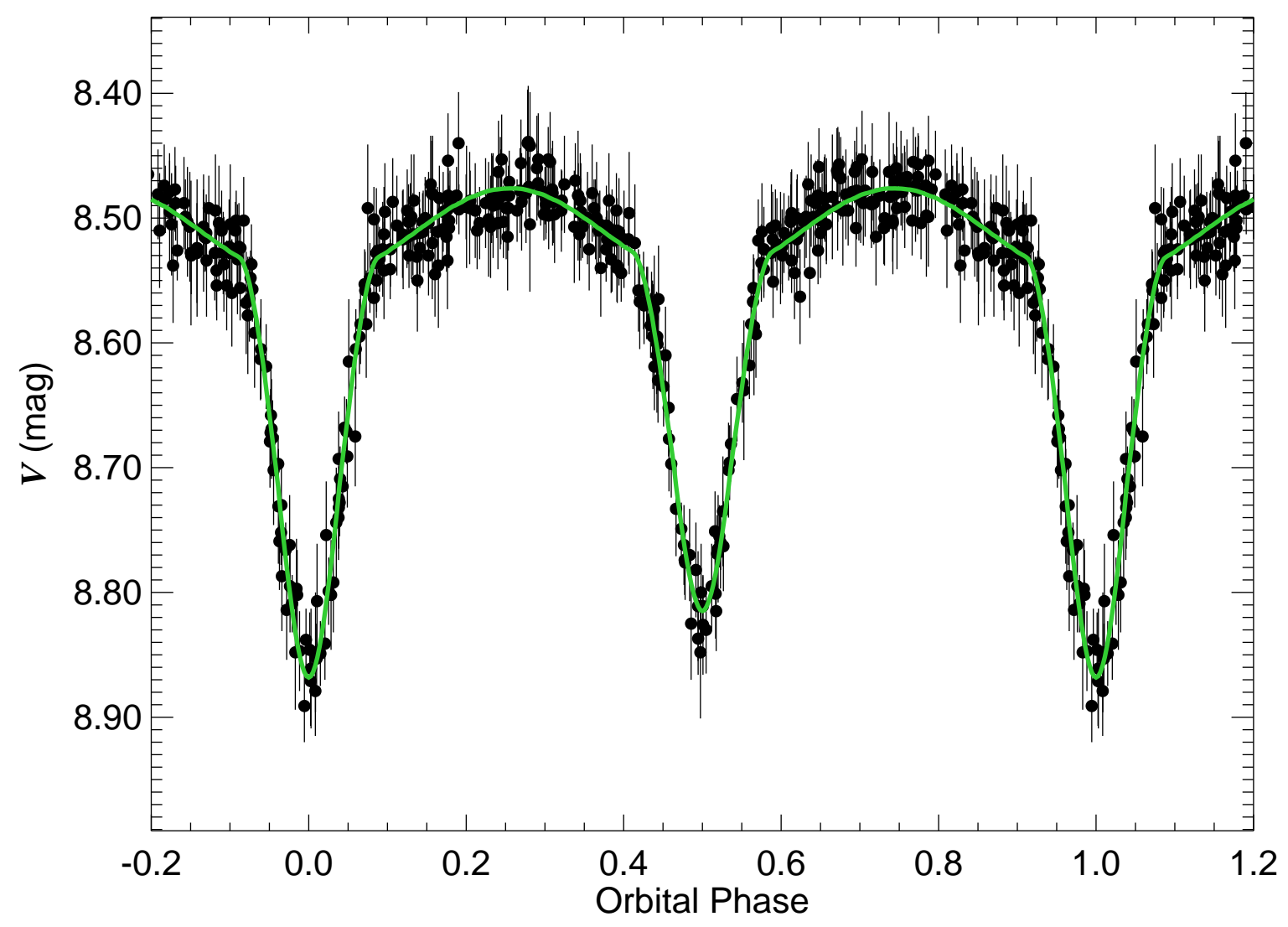

Fig. 32.- The ASAS $V$-band light curve for ASAS 073348-0940.9 (HD 60389). Filled circles with lines represent data with associated uncertainties. The best fit orbital solution listed in Table 2 is shown as a solid line passing through the data. 


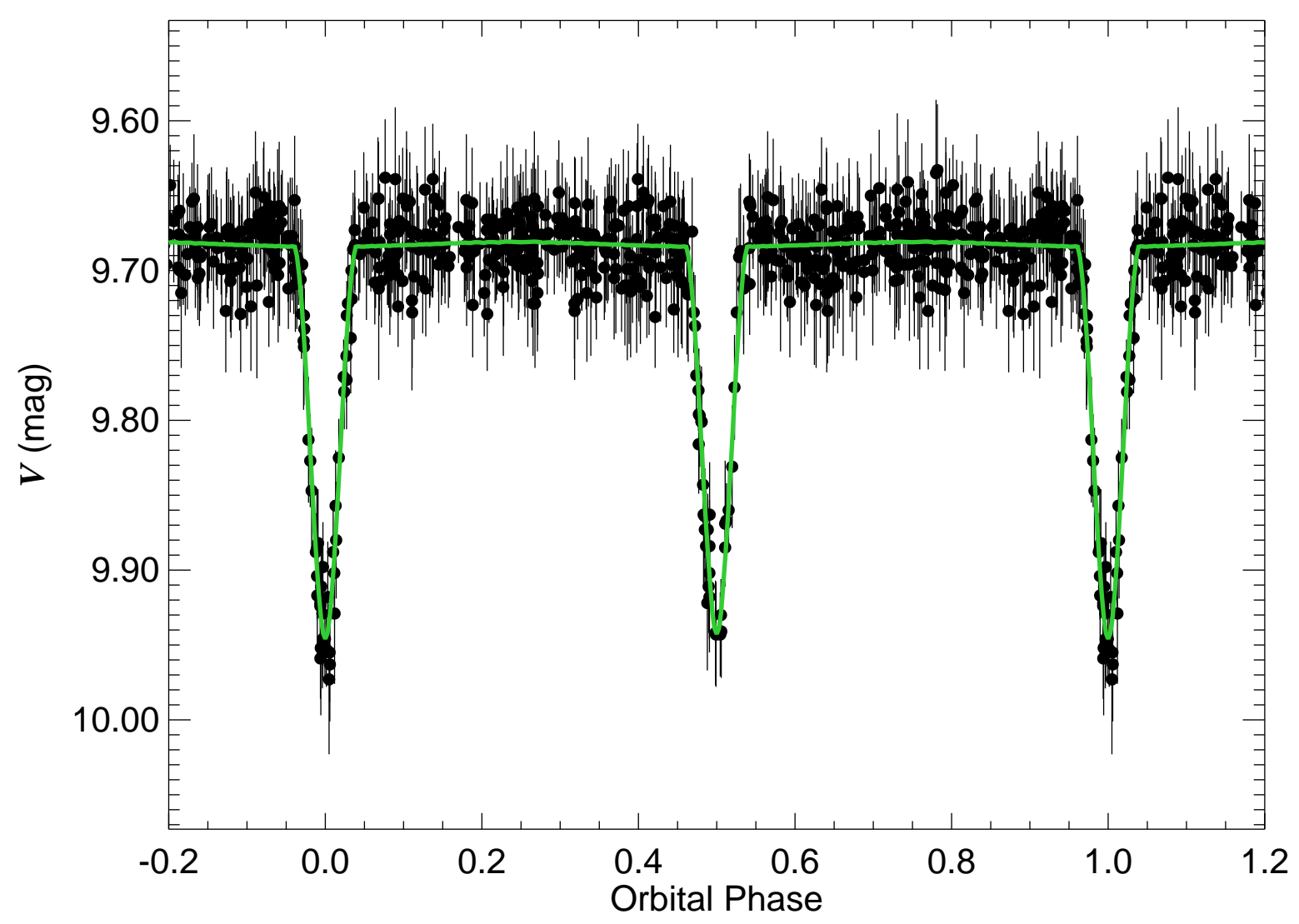

Fig. 33.- The ASAS $V$-band light curve for ASAS 074355-2517.9 (HD 62607). Filled circles with lines represent data with associated uncertainties. The best fit orbital solution listed in Table 2 is shown as a solid line passing through the data. 


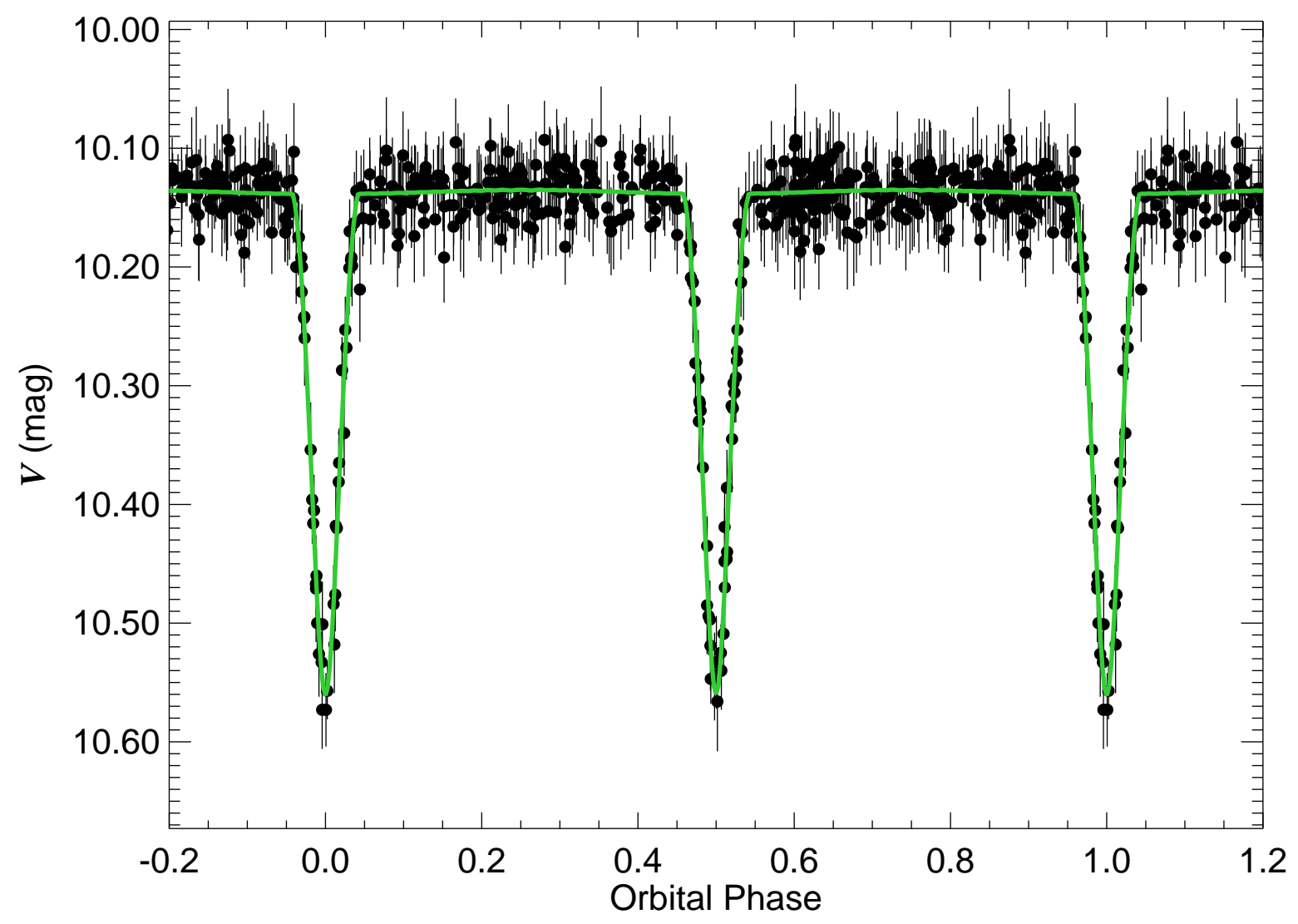

Fig. 34.- The ASAS $V$-band light curve for ASAS 074714-0519.8 (HD 63141). Filled circles with lines represent data with associated uncertainties. The best fit orbital solution listed in Table 2 is shown as a solid line passing through the data. 


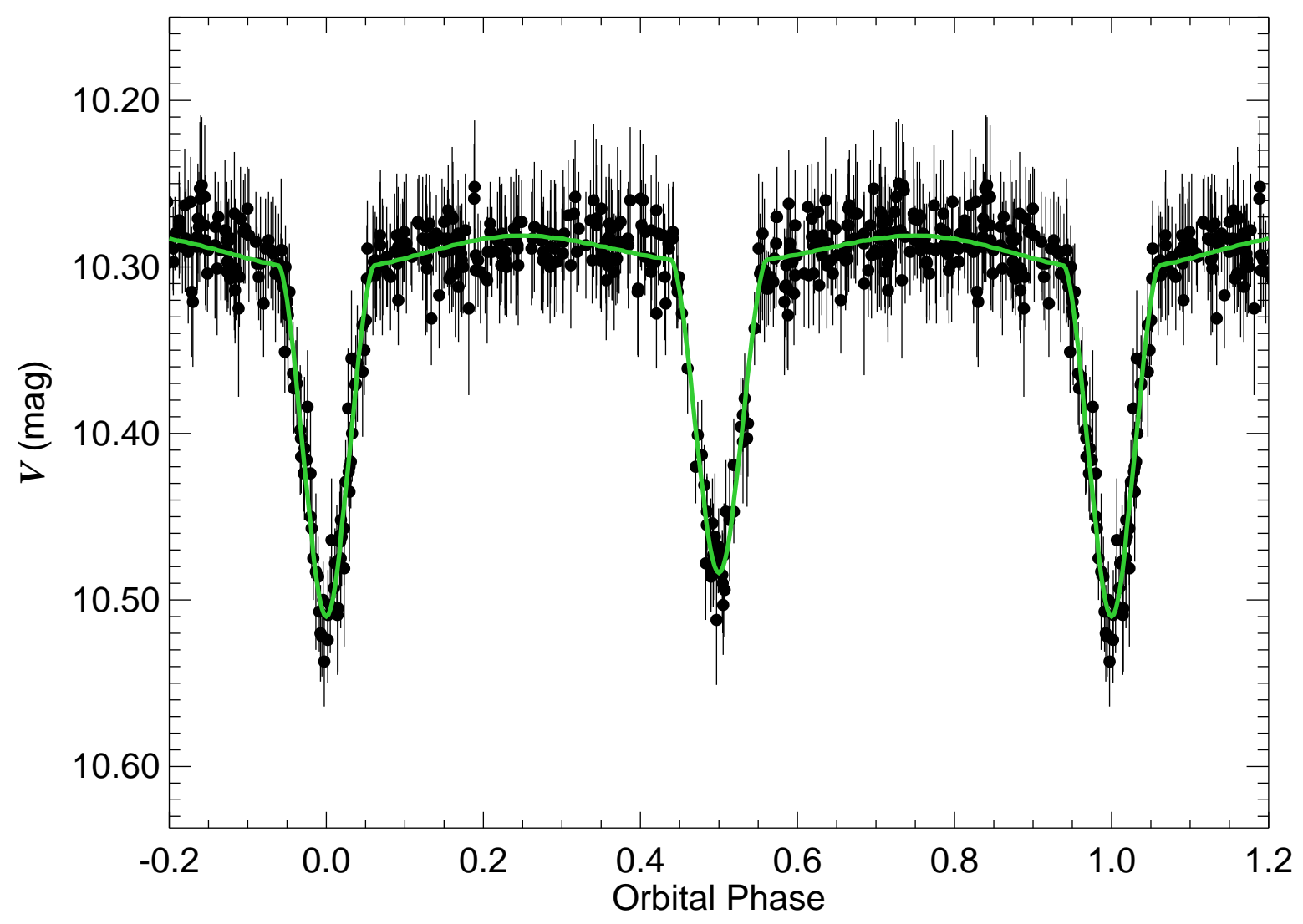

Fig. 35.- The ASAS $V$-band light curve for ASAS 074928-0721.6 (BD -06 2317). Filled circles with lines represent data with associated uncertainties. The best fit orbital solution listed in Table 2 is shown as a solid line passing through the data. 


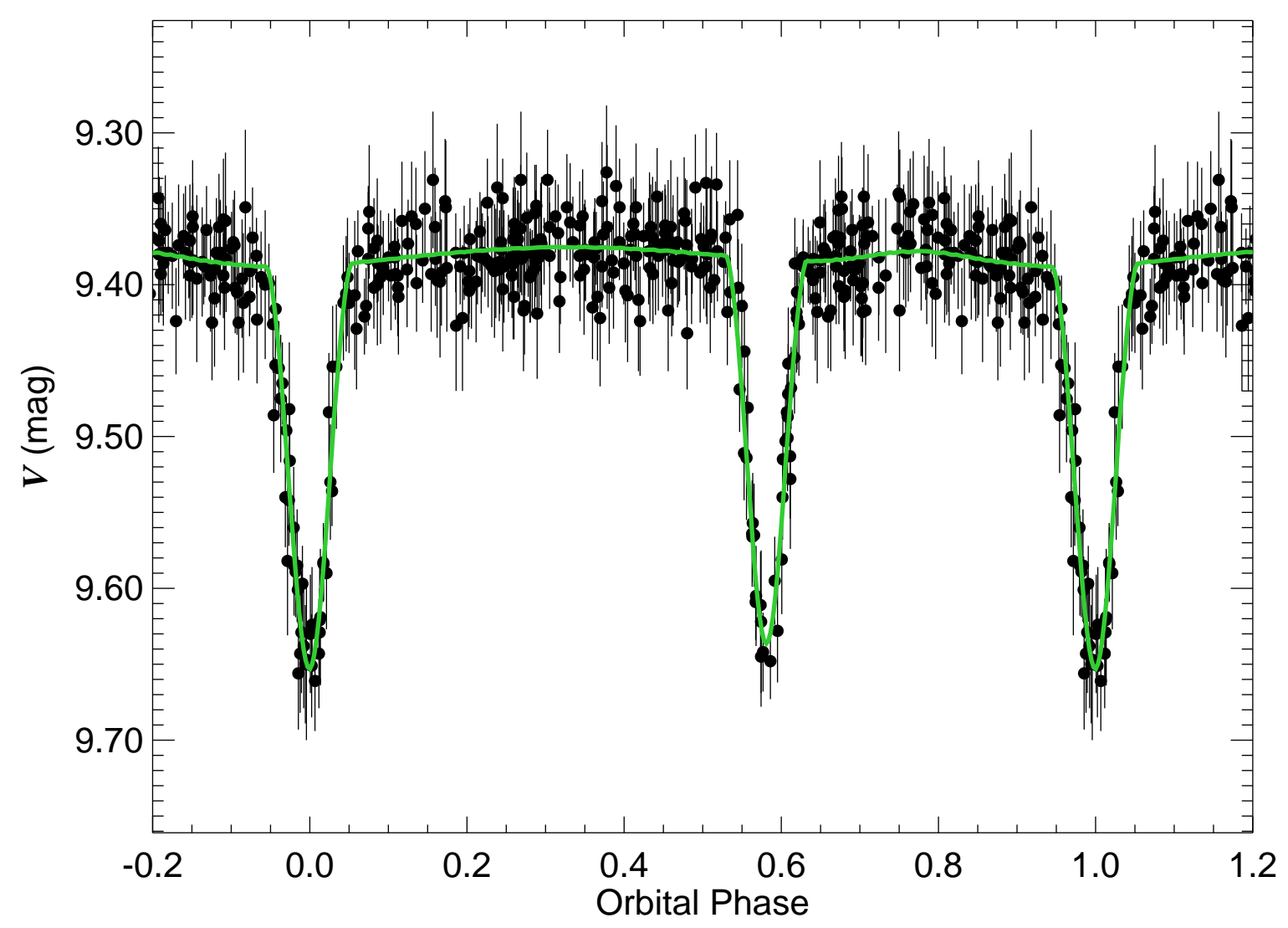

Fig. 36.- The ASAS $V$-band light curve for ASAS 075052+0048.0 (HD 63818). Filled circles with lines represent data with associated uncertainties. The best fit orbital solution listed in Table 2 is shown as a solid line passing through the data. 


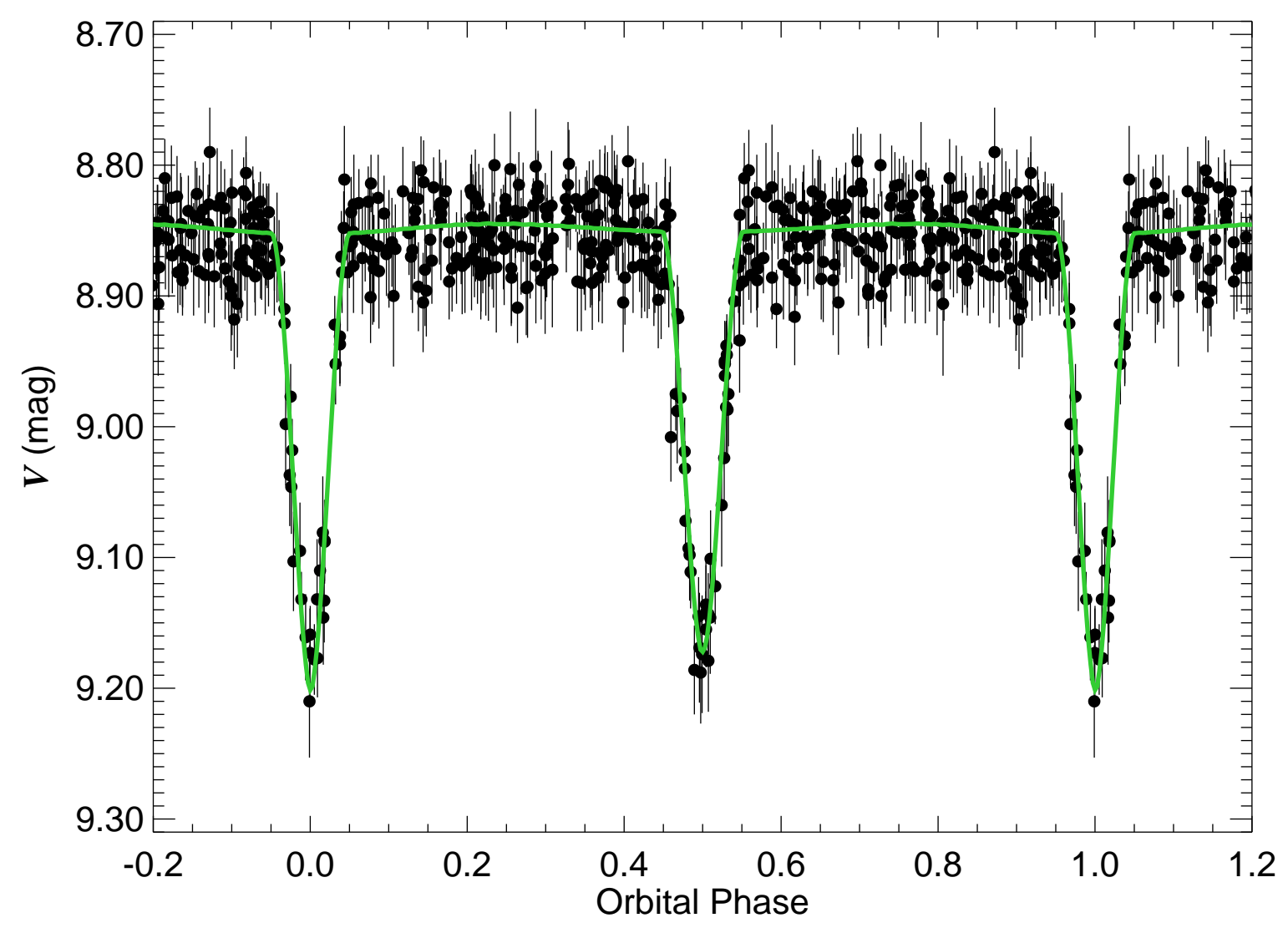

Fig. 37.- The ASAS $V$-band light curve for ASAS 080617-0426.8 (V871 Mon). Filled circles with lines represent data with associated uncertainties. The best fit orbital solution listed in Table 2 is shown as a solid line passing through the data. 


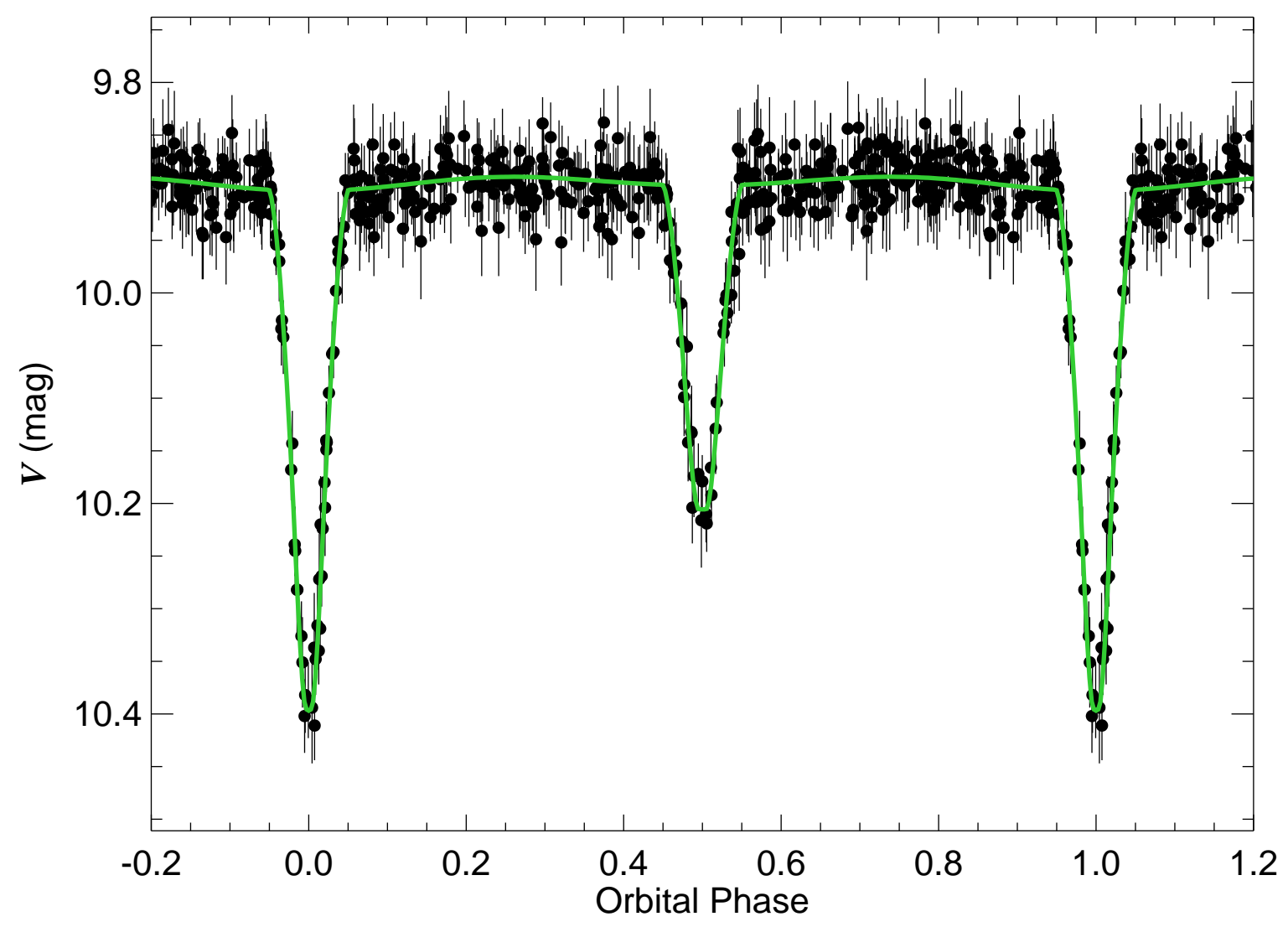

Fig. 38. - The ASAS $V$-band light curve for ASAS 081749-2659.7 (HD 69797). Filled circles with lines represent data with associated uncertainties. The best fit orbital solution listed in Table 2 is shown as a solid line passing through the data. 


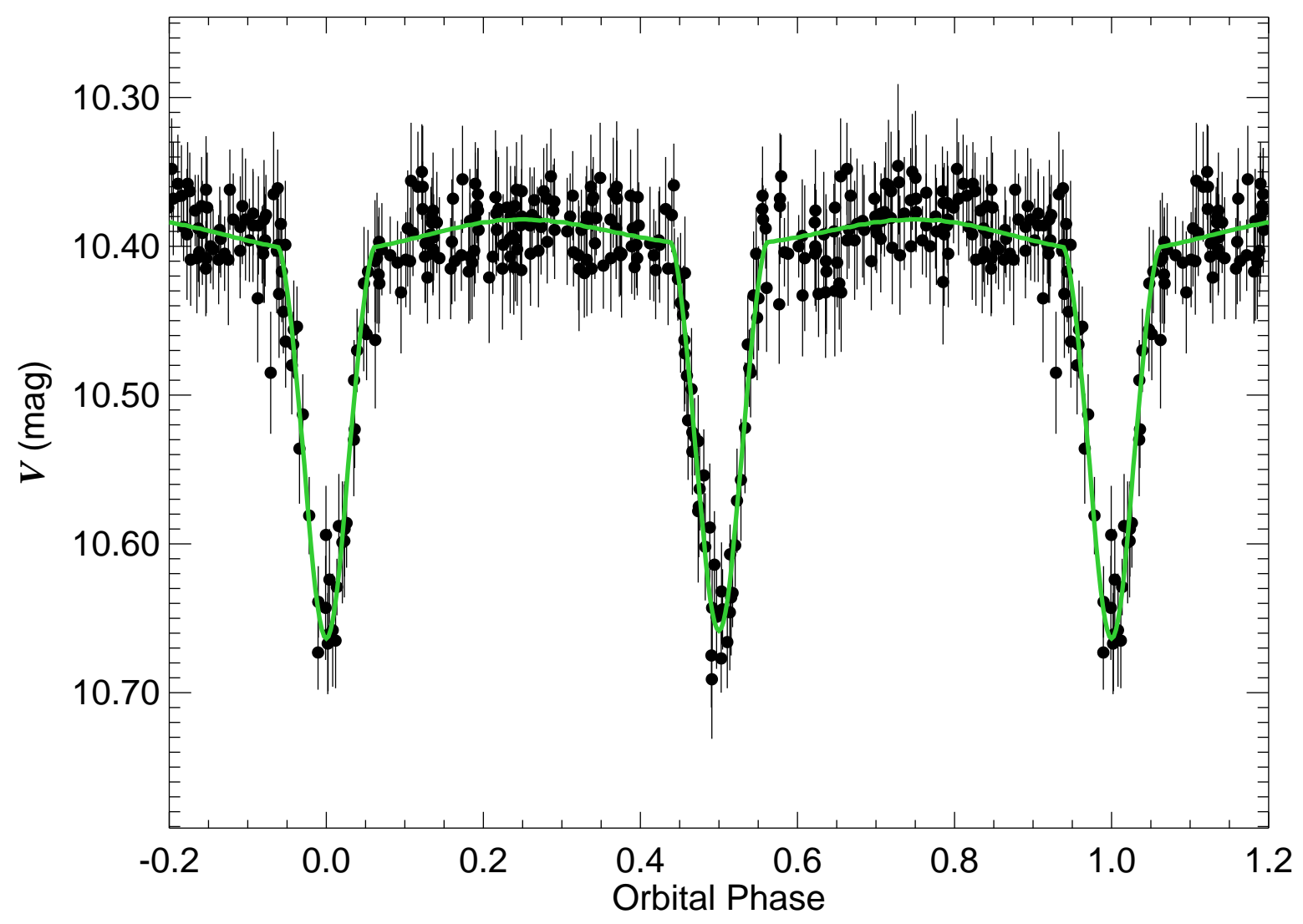

Fig. 39. - The ASAS $V$-band light curve for ASAS 083245+0247.3 (BD +03 2001). Filled circles with lines represent data with associated uncertainties. The best fit orbital solution listed in Table 2 is shown as a solid line passing through the data. 


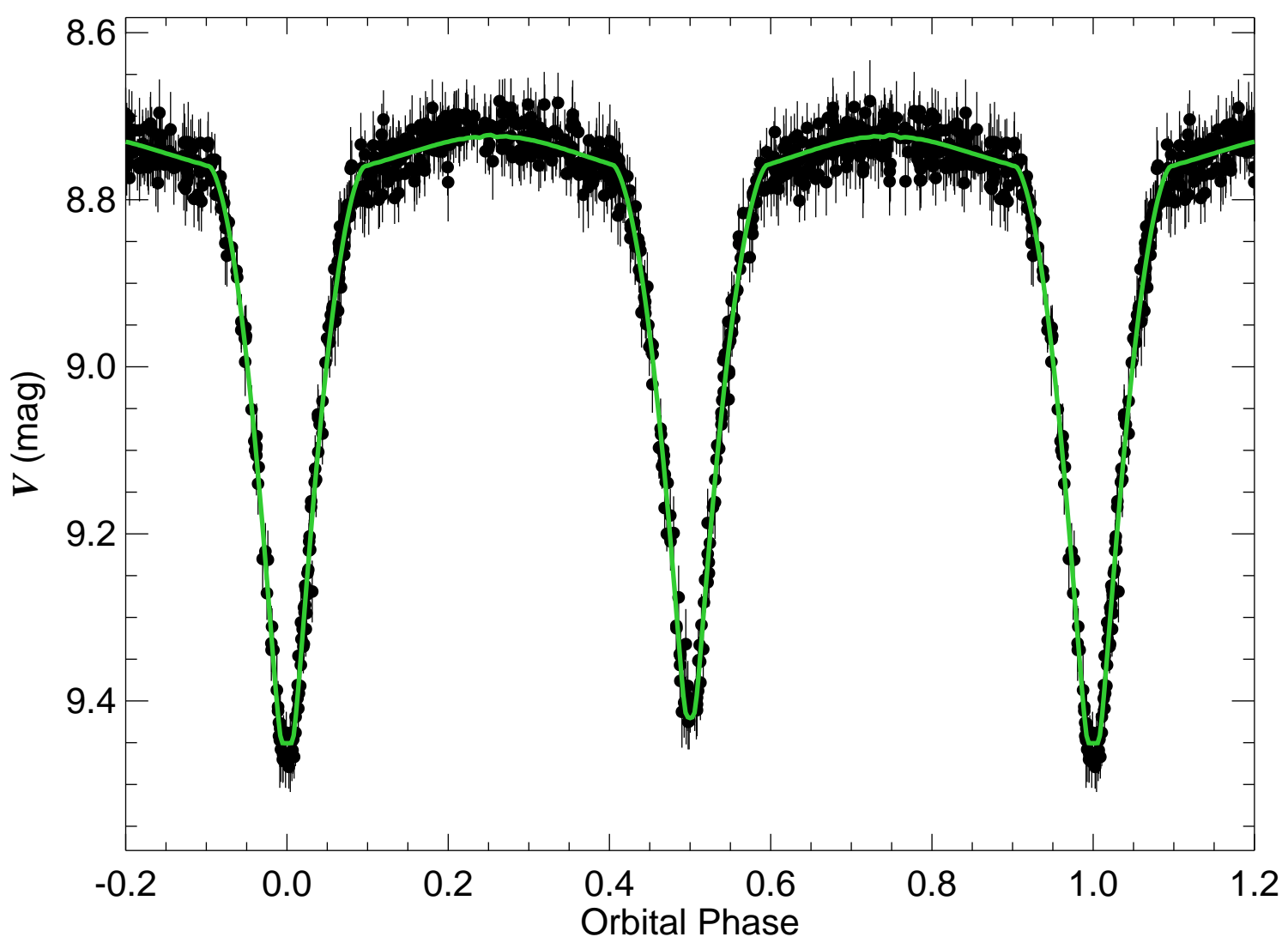

Fig. 40.- The ASAS $V$-band light curve for ASAS 084831-2609.8 (TT Pyx). Filled circles with lines represent data with associated uncertainties. The best fit orbital solution listed in Table 2 is shown as a solid line passing through the data. 


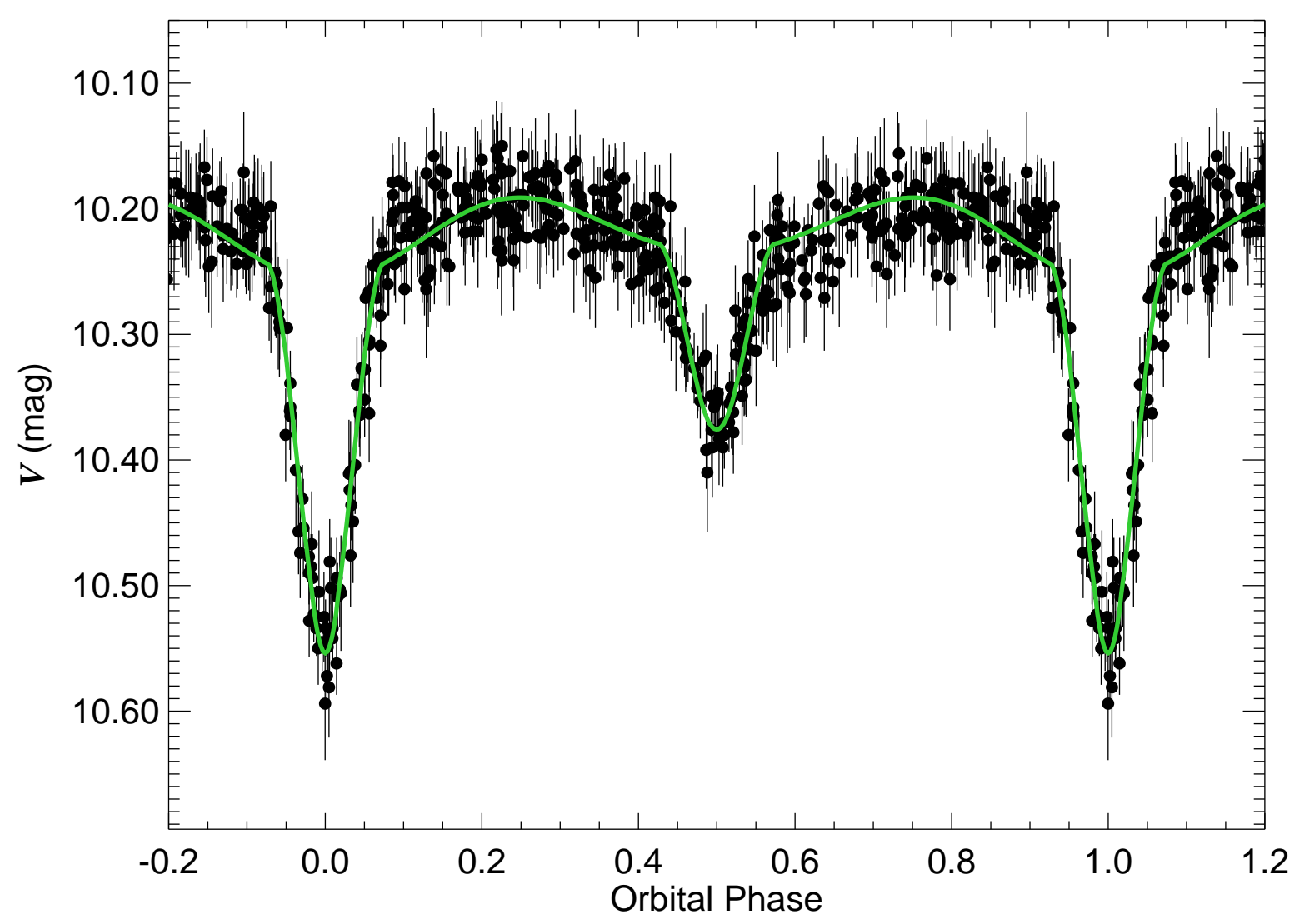

Fig. 41.- The ASAS $V$-band light curve for ASAS 101120-1956.3 (HD 88409). Filled circles with lines represent data with associated uncertainties. The best fit orbital solution listed in Table 2 is shown as a solid line passing through the data. 


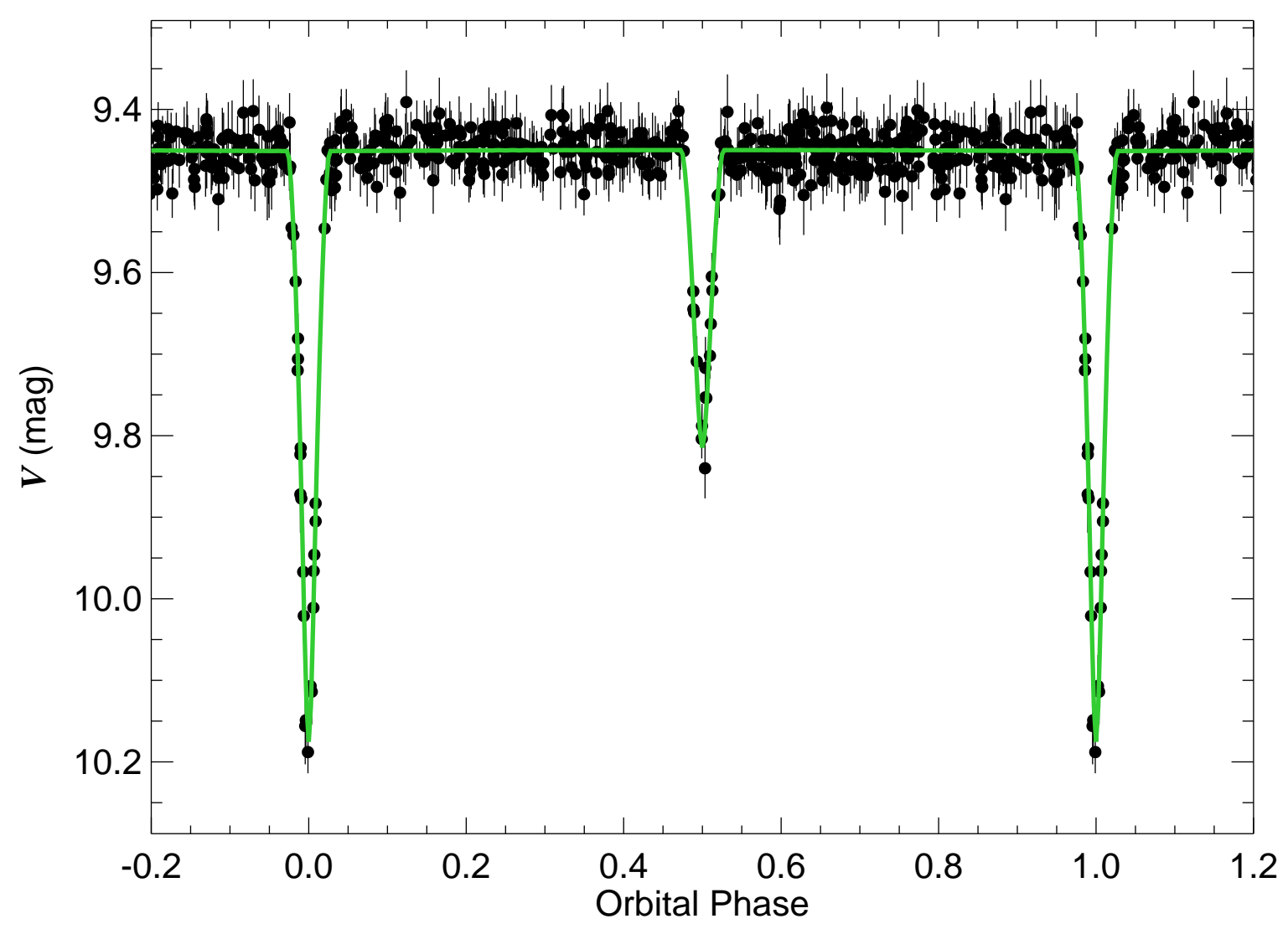

Fig. 42.- The ASAS V-band light curve for ASAS 135949-2745.5 (HD 122026). Filled circles with lines represent data with associated uncertainties. The best fit orbital solution listed in Table 2 is shown as a solid line passing through the data. 


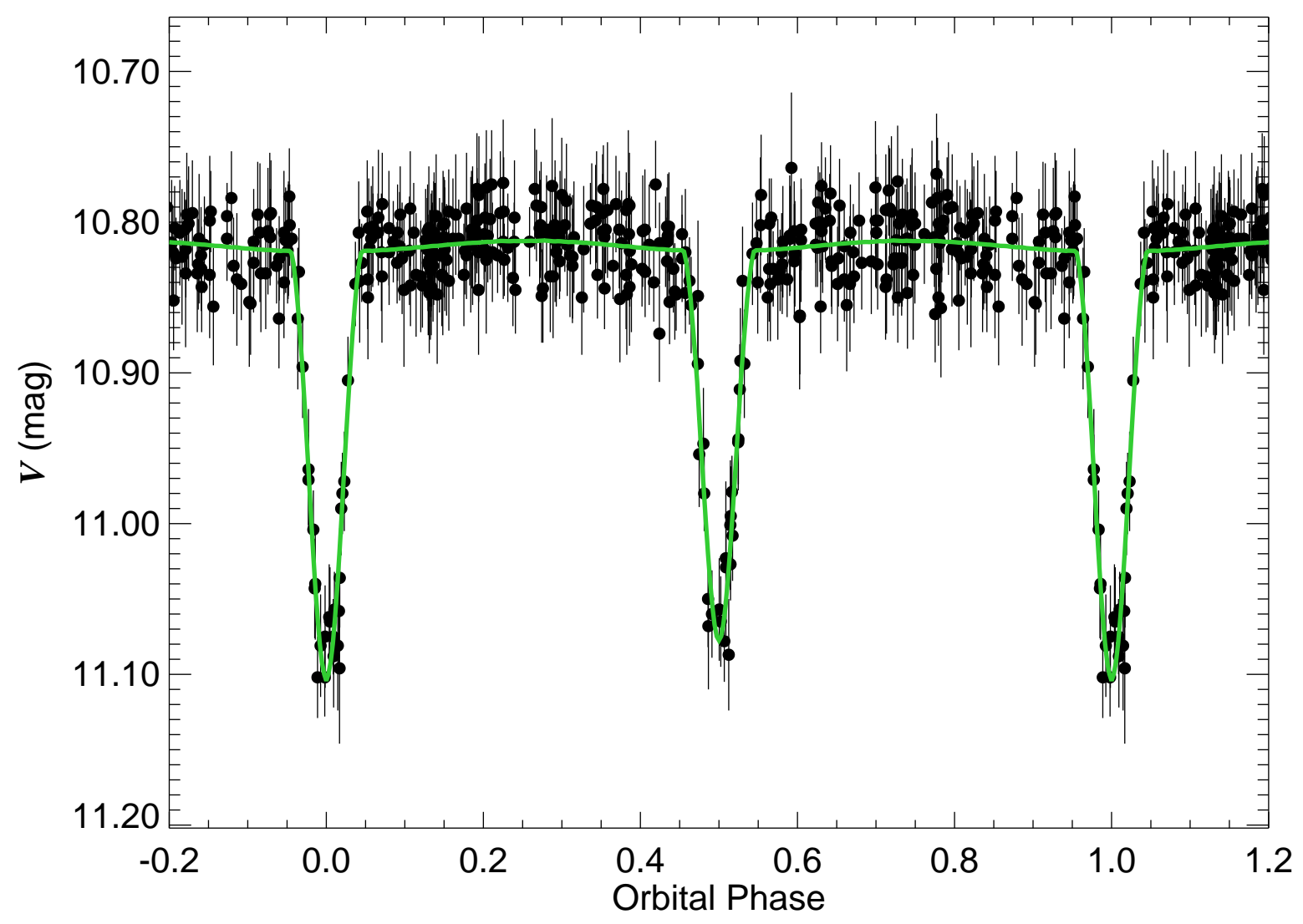

Fig. 43.- The ASAS $V$-band light curve for ASAS 160851-2351.0 (TYC 6780-1523-1). Filled circles with lines represent data with associated uncertainties. The best fit orbital solution listed in Table 2 is shown as a solid line passing through the data. 


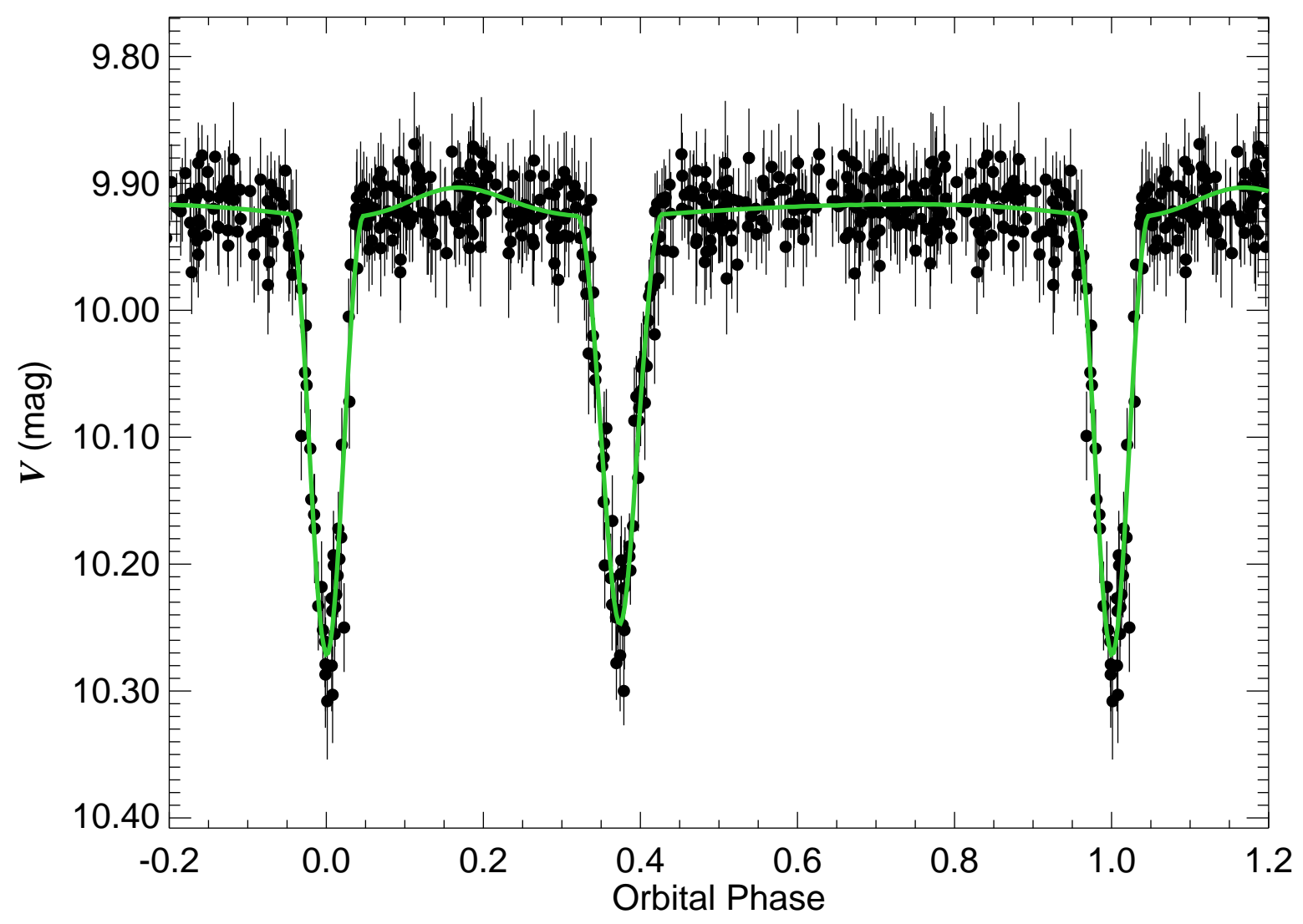

Fig. 44.- The ASAS V-band light curve for ASAS 165354-1301.9 (HD 152451). Filled circles with lines represent data with associated uncertainties. The best fit orbital solution listed in Table 2 is shown as a solid line passing through the data. 


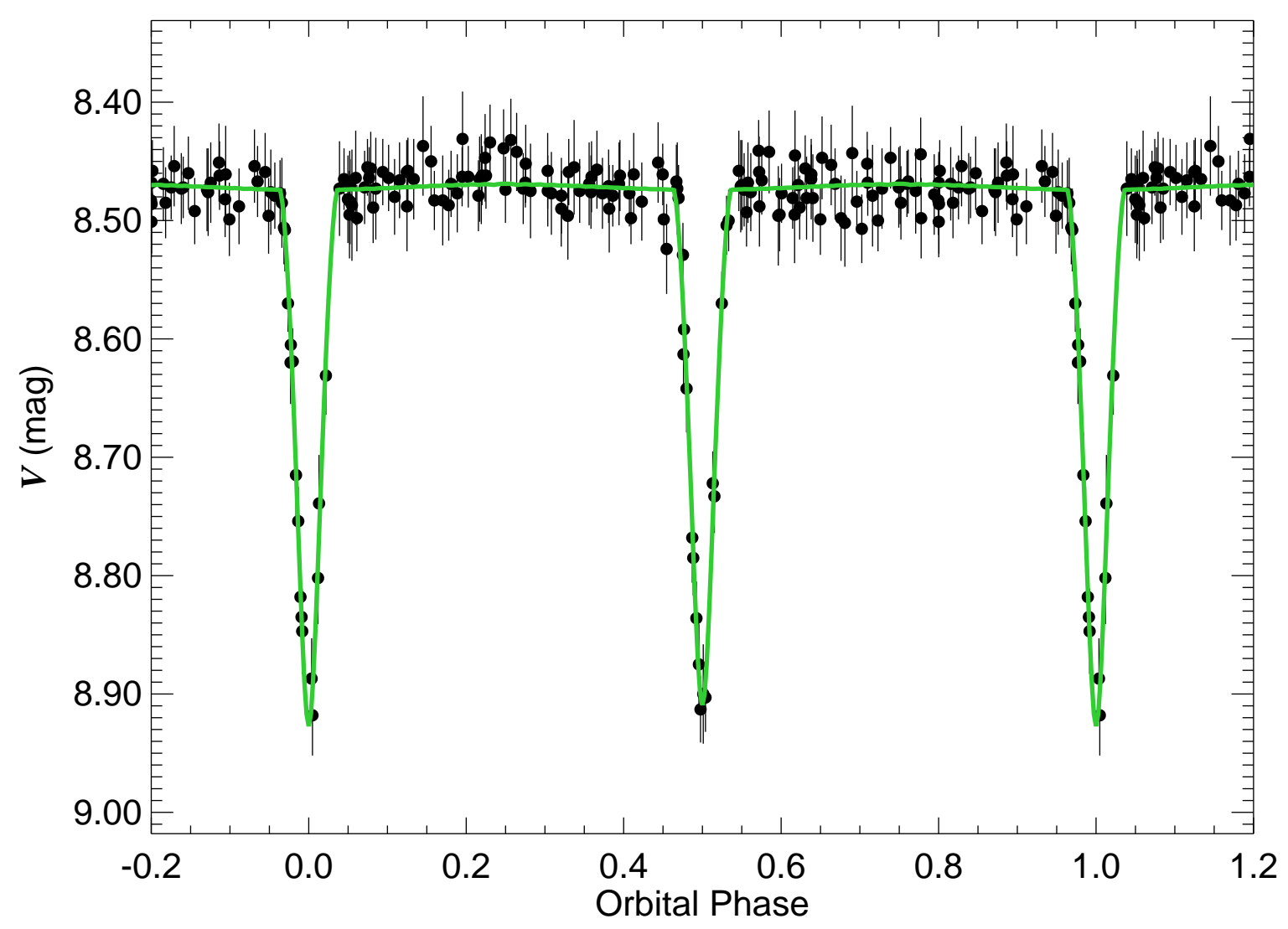

Fig. 45.- The ASAS $V$-band light curve for ASAS 170158+2348.4 (HD 154010). Filled circles with lines represent data with associated uncertainties. The best fit orbital solution listed in Table 2 is shown as a solid line passing through the data. 


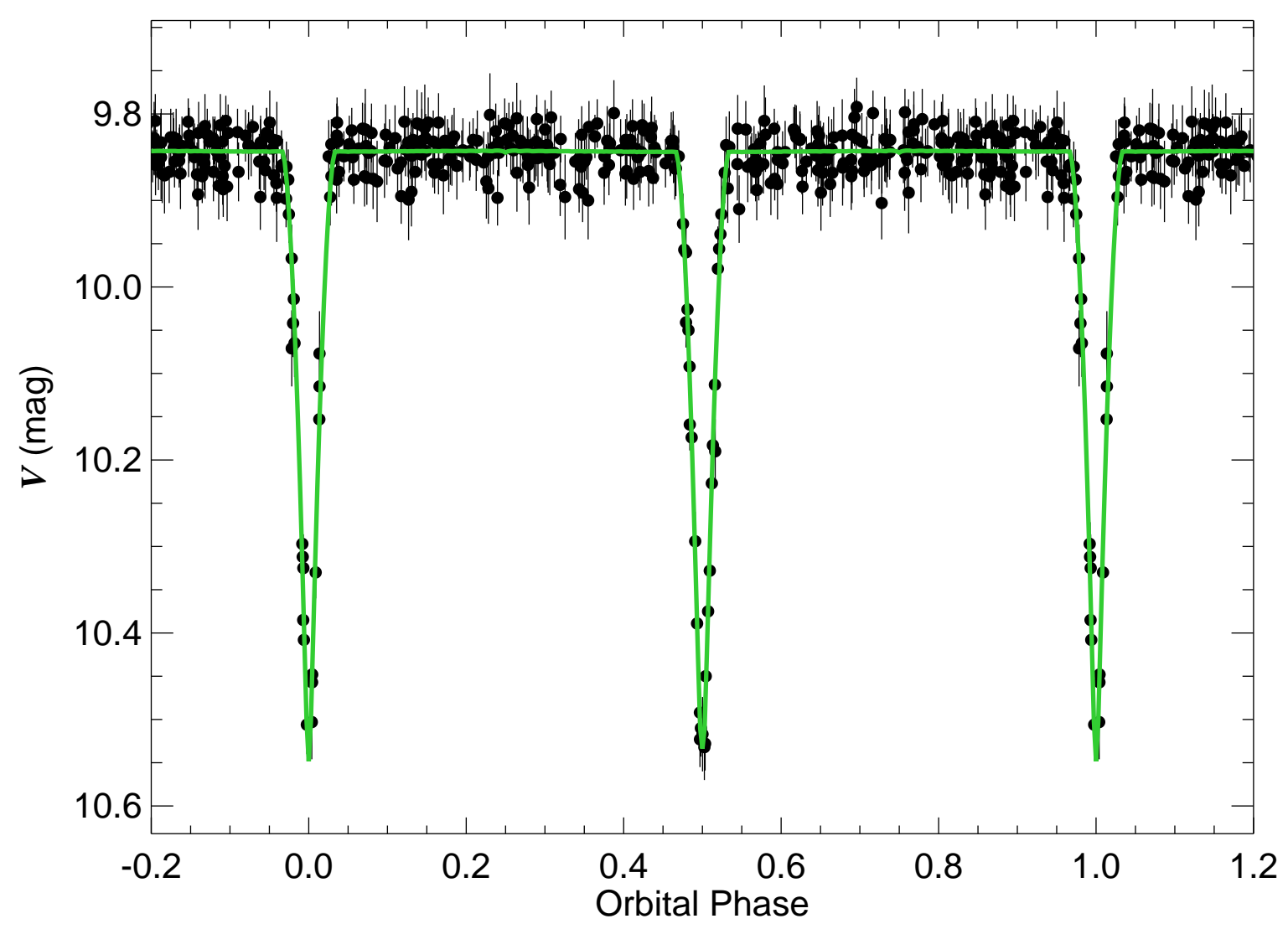

Fig. 46.- The ASAS V-band light curve for ASAS 173421-1836.3 (HD 159246). Filled circles with lines represent data with associated uncertainties. The best fit orbital solution listed in Table 2 is shown as a solid line passing through the data. 


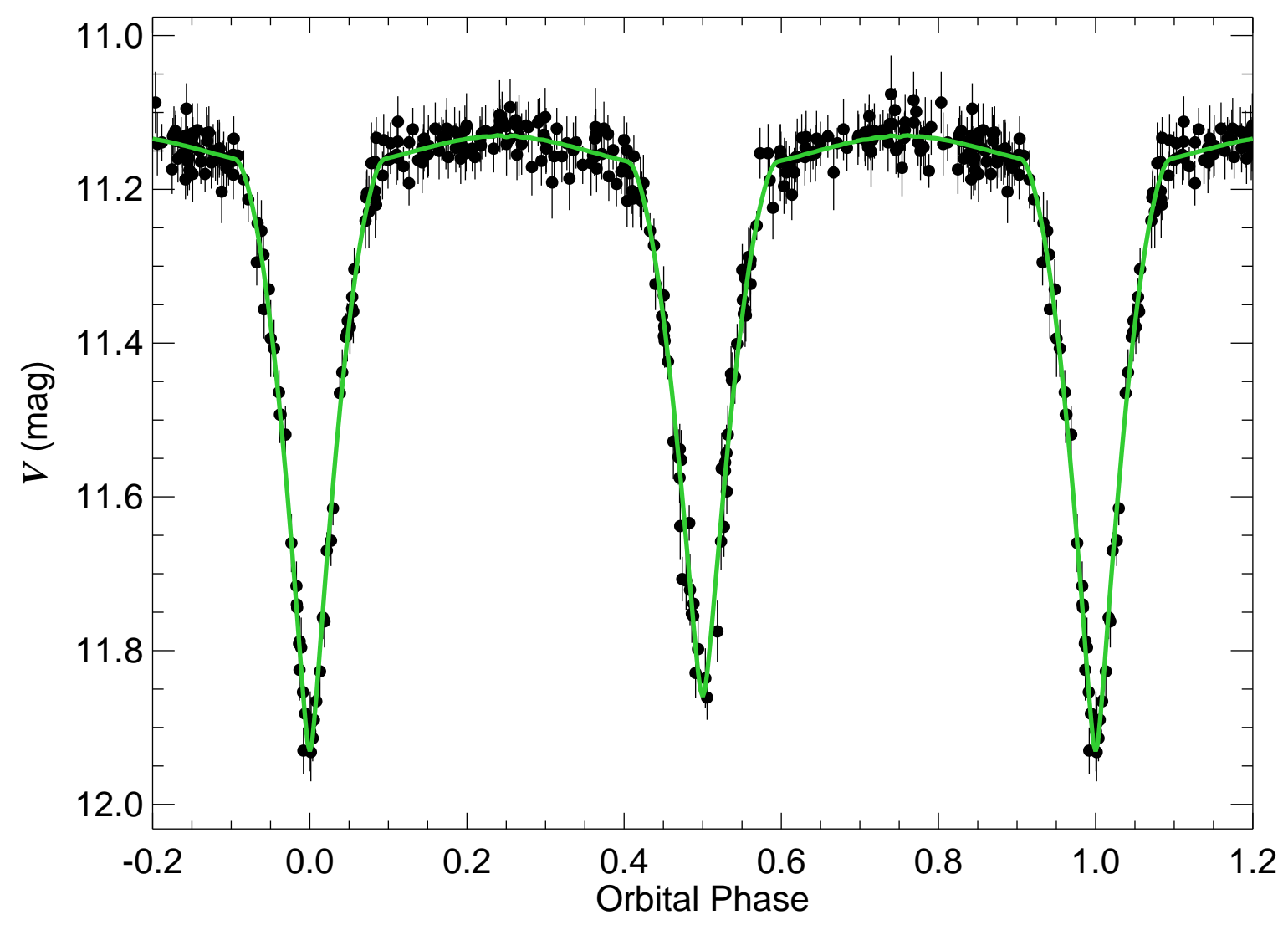

Fig. 47.- The ASAS $V$-band light curve for ASAS 174104+0747.1 (V506 Oph). Filled circles with lines represent data with associated uncertainties. The best fit orbital solution listed in Table 2 is shown as a solid line passing through the data. 


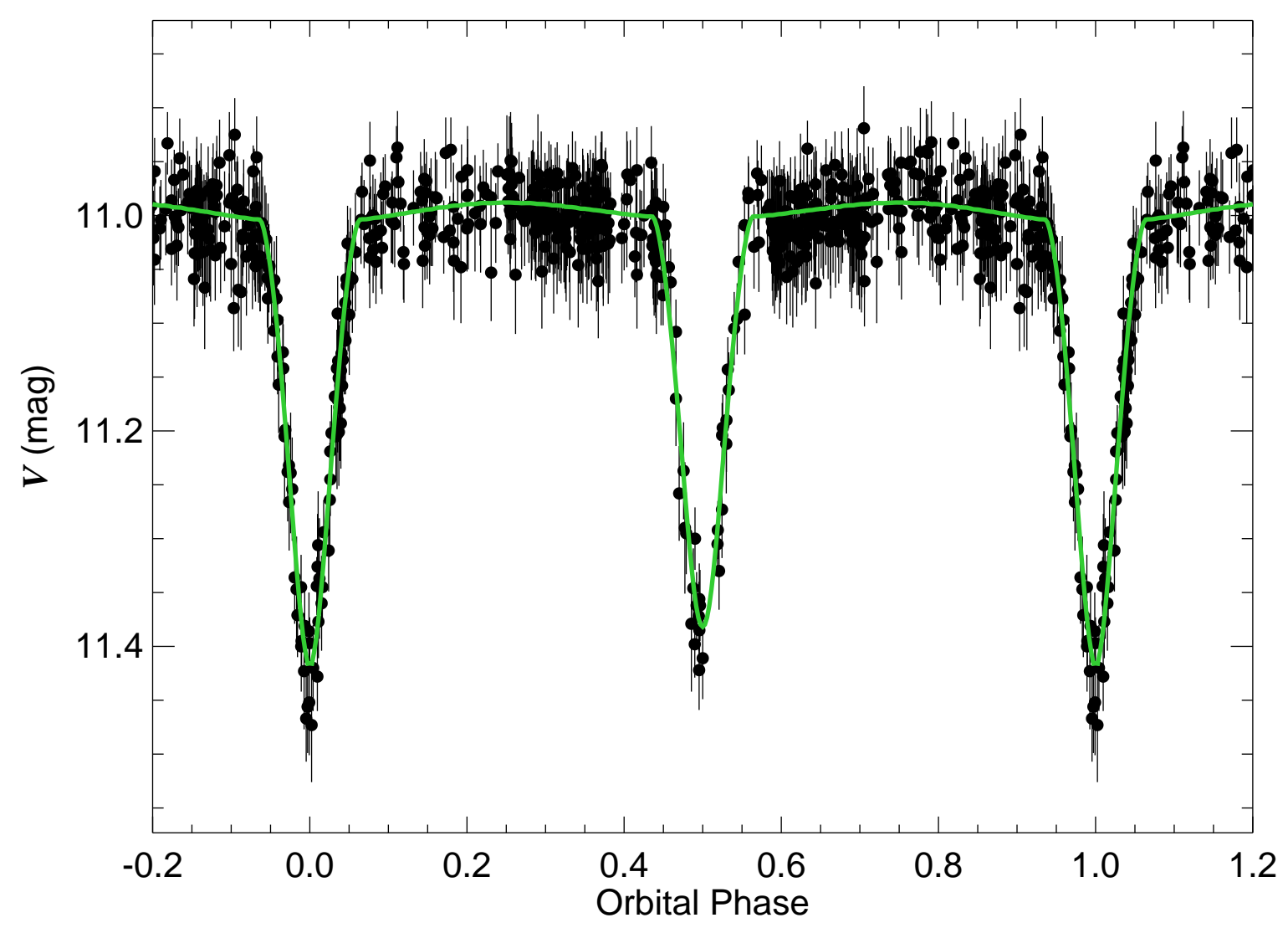

Fig. 48.- The ASAS V-band light curve for ASAS 175659-2012.2 (HD 312444). Filled circles with lines represent data with associated uncertainties. The best fit orbital solution listed in Table 2 is shown as a solid line passing through the data. 


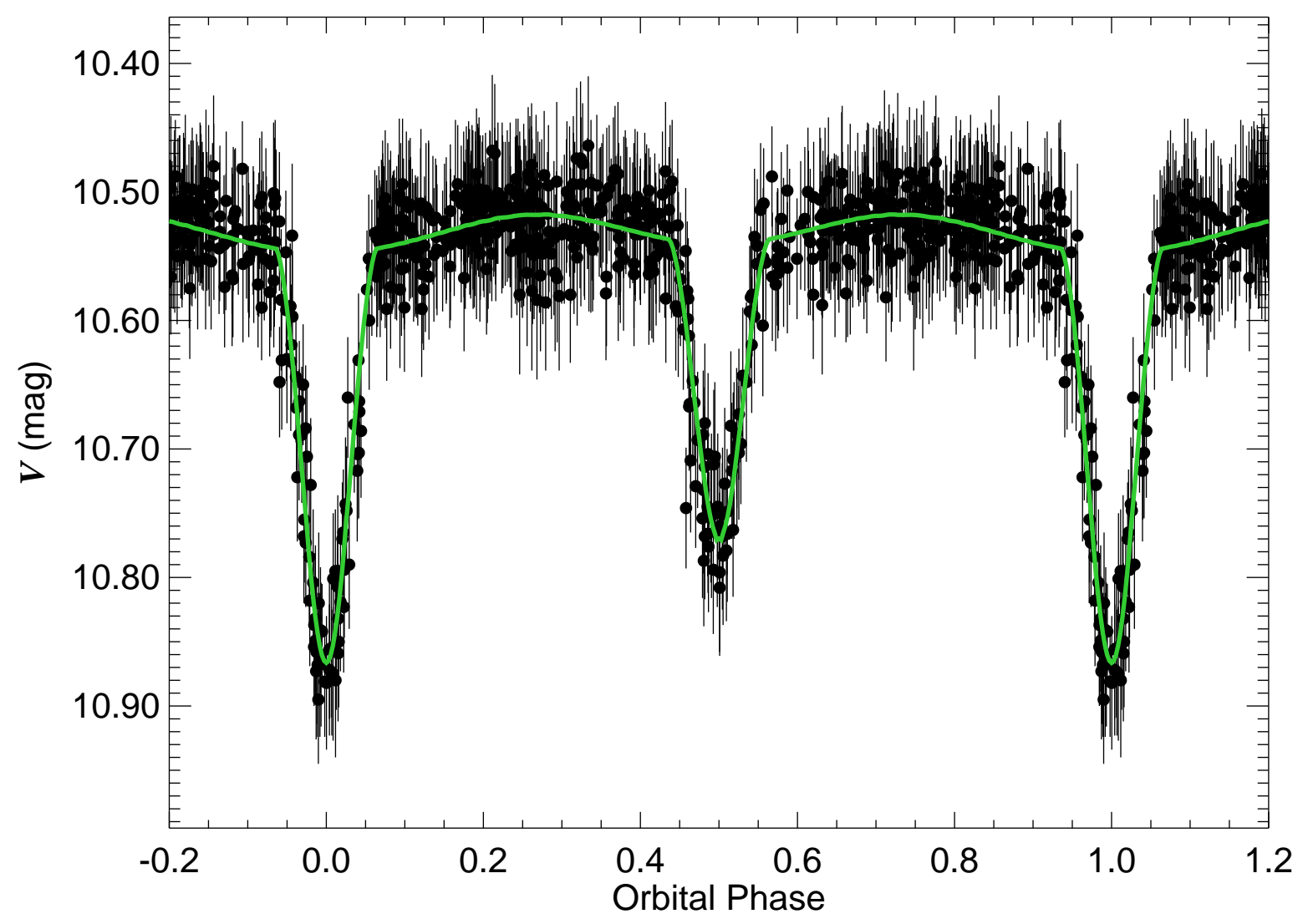

Fig. 49.- The ASAS $V$-band light curve for ASAS 175859-2323.1 (HD 313508). Filled circles with lines represent data with associated uncertainties. The best fit orbital solution listed in Table 2 is shown as a solid line passing through the data. 


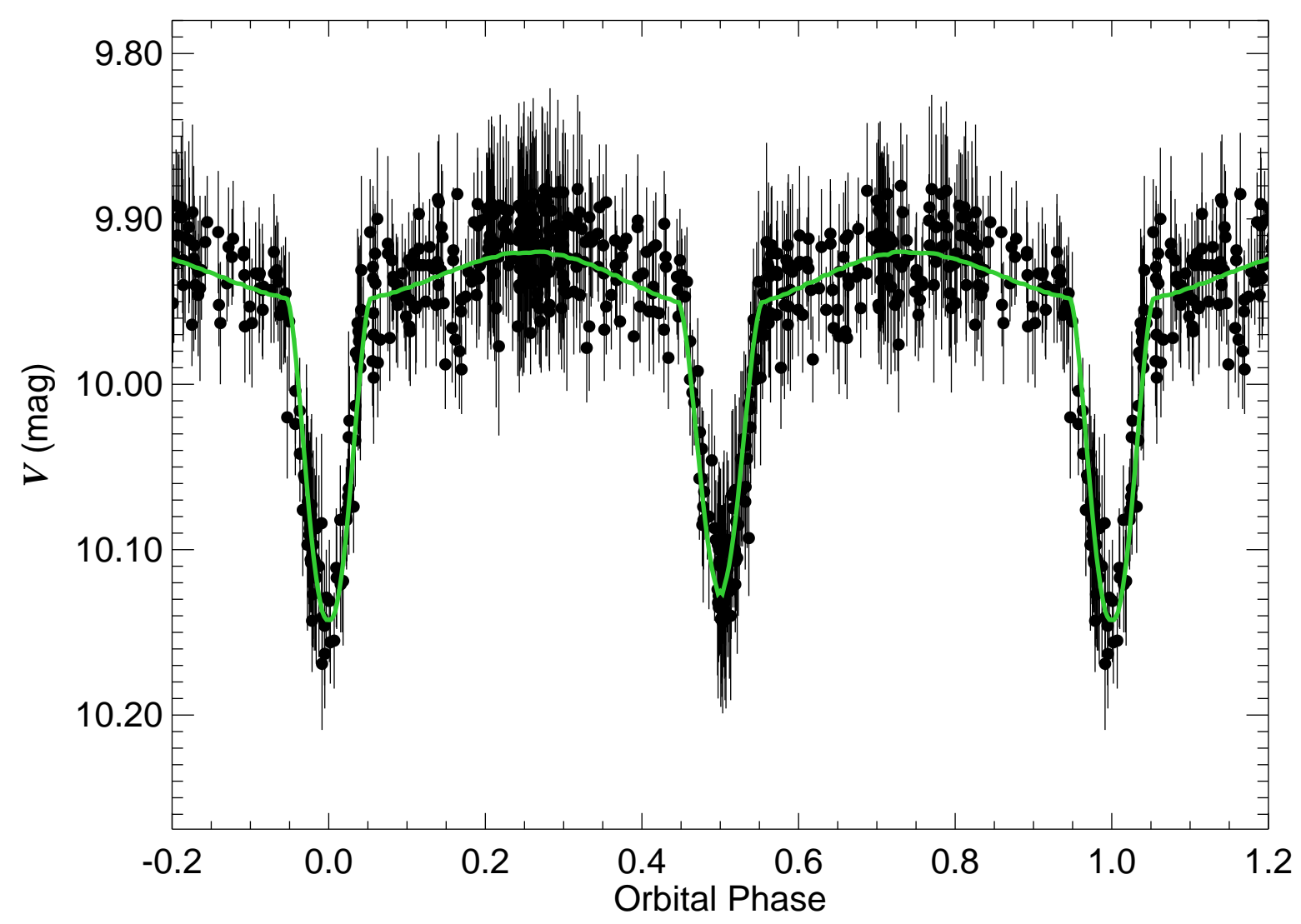

Fig. 50.- The ASAS $V$-band light curve for ASAS 180903-1824.5 (HD 165890). Filled circles with lines represent data with associated uncertainties. The best fit orbital solution listed in Table 2 is shown as a solid line passing through the data. 


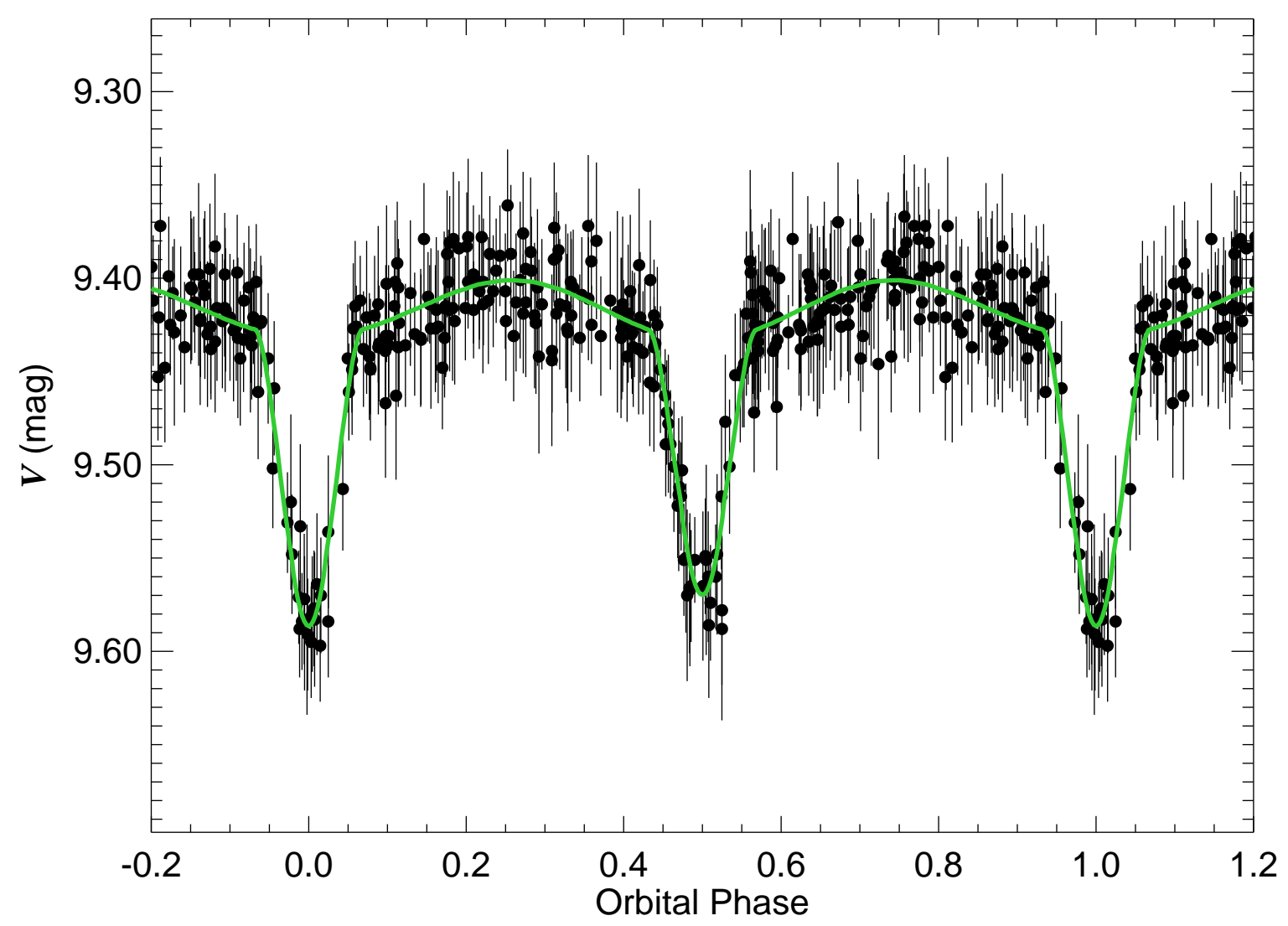

Fig. 51.- The ASAS $V$-band light curve for ASAS 181025+0047.7 (HD 166383). Filled circles with lines represent data with associated uncertainties. The best fit orbital solution listed in Table 2 is shown as a solid line passing through the data. 


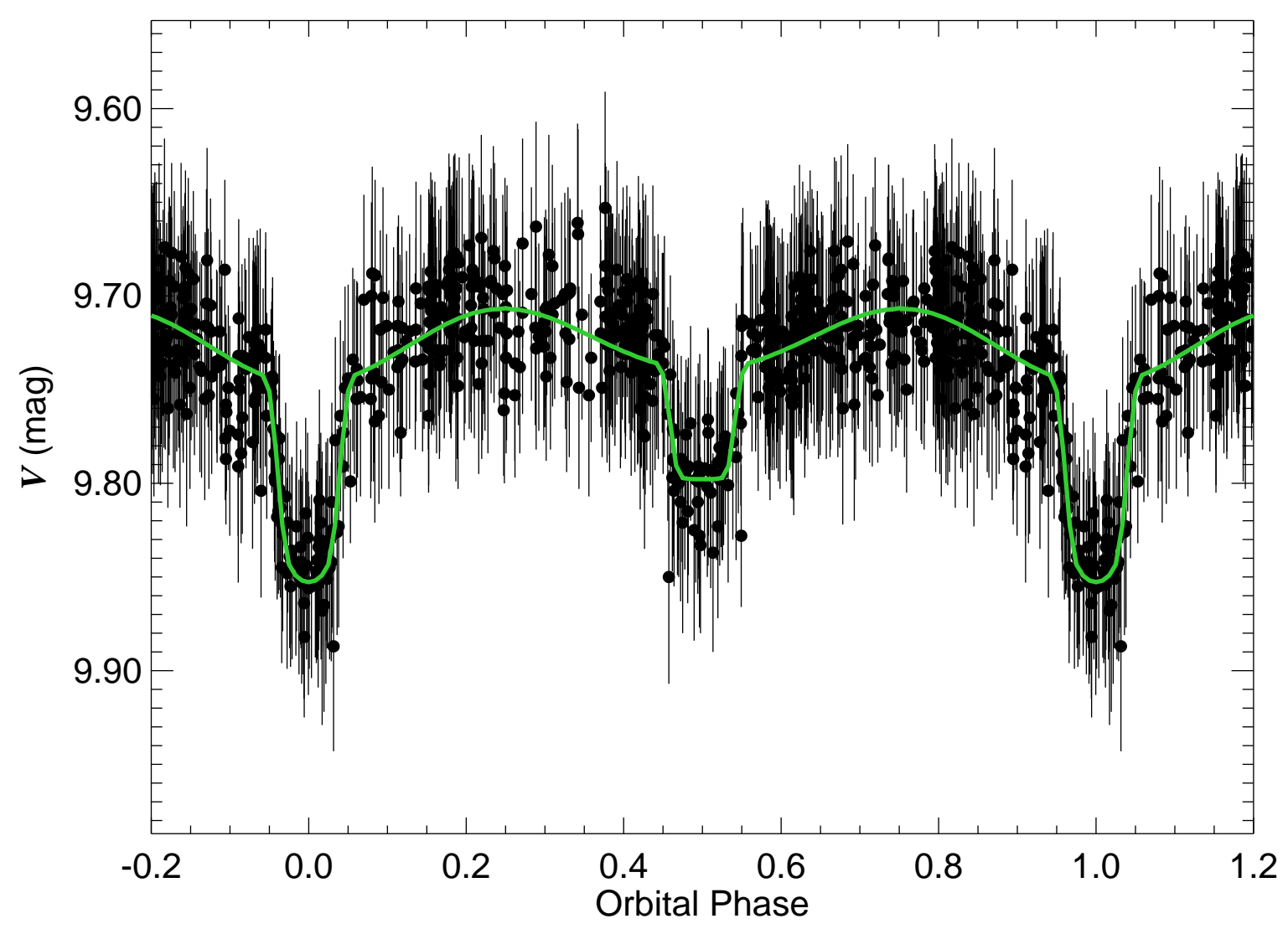

Fig. 52.- The ASAS $V$-band light curve for ASAS 181328-2214.3 (HD 166851). Filled circles with lines represent data with associated uncertainties. The best fit orbital solution listed in Table 2 is shown as a solid line passing through the data. 


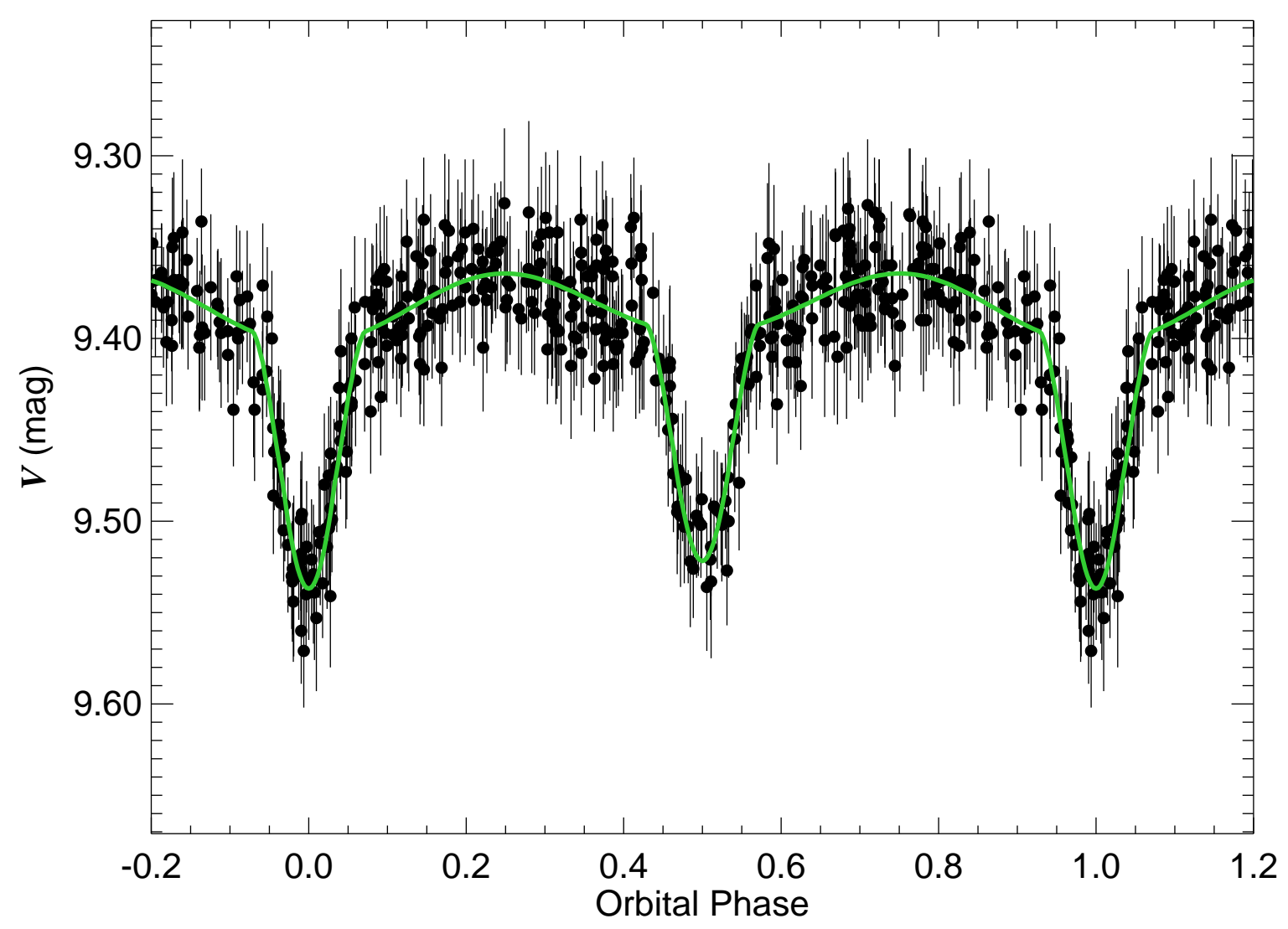

Fig. 53.- The ASAS $V$-band light curve for ASAS 181909-1410.0 (HD 168207). Filled circles with lines represent data with associated uncertainties. The best fit orbital solution listed in Table 2 is shown as a solid line passing through the data. 


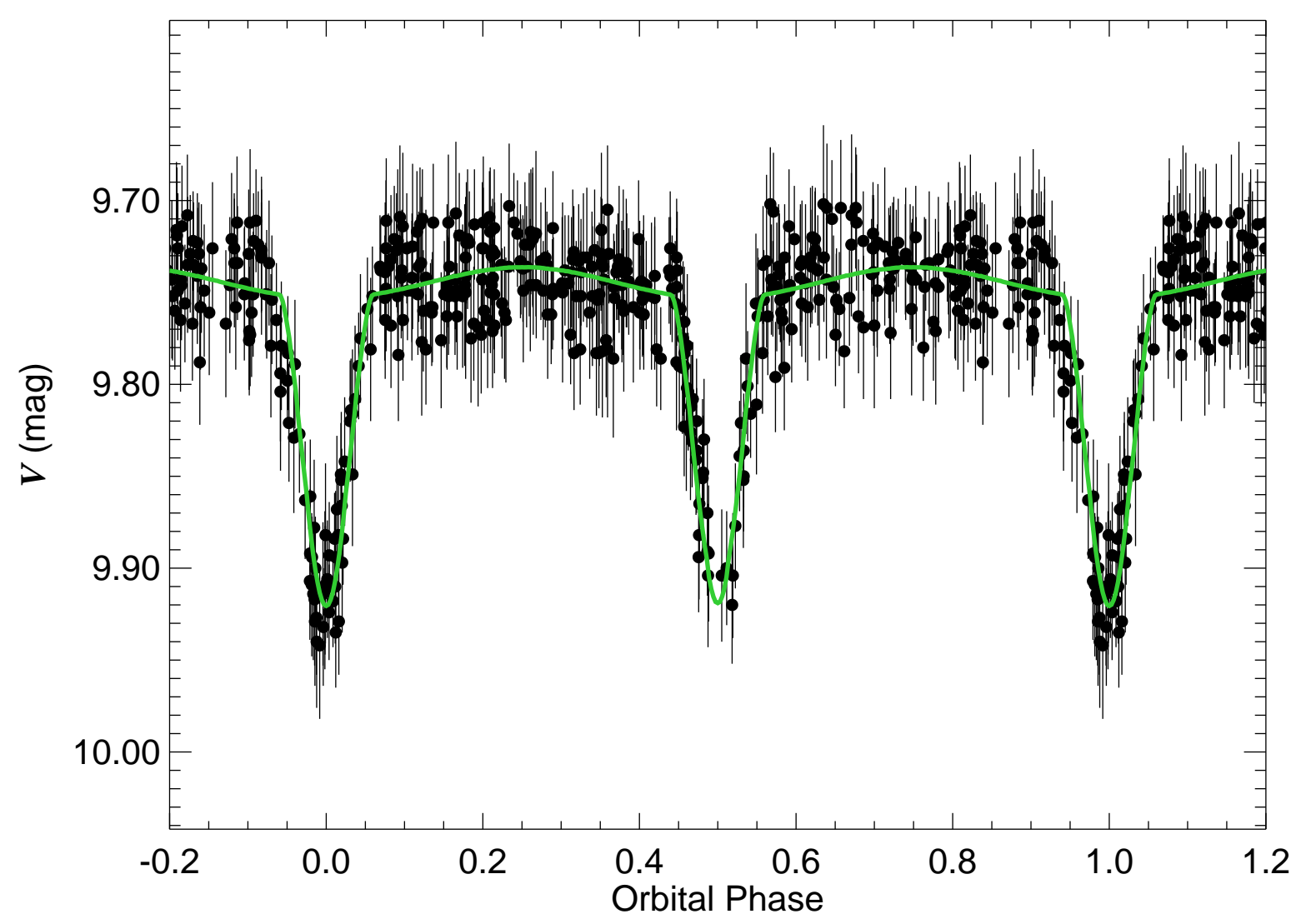

Fig. 54.- The ASAS V-band light curve for ASAS 183129-1918.8 (BD - 19 5039). Filled circles with lines represent data with associated uncertainties. The best fit orbital solution listed in Table 2 is shown as a solid line passing through the data. 


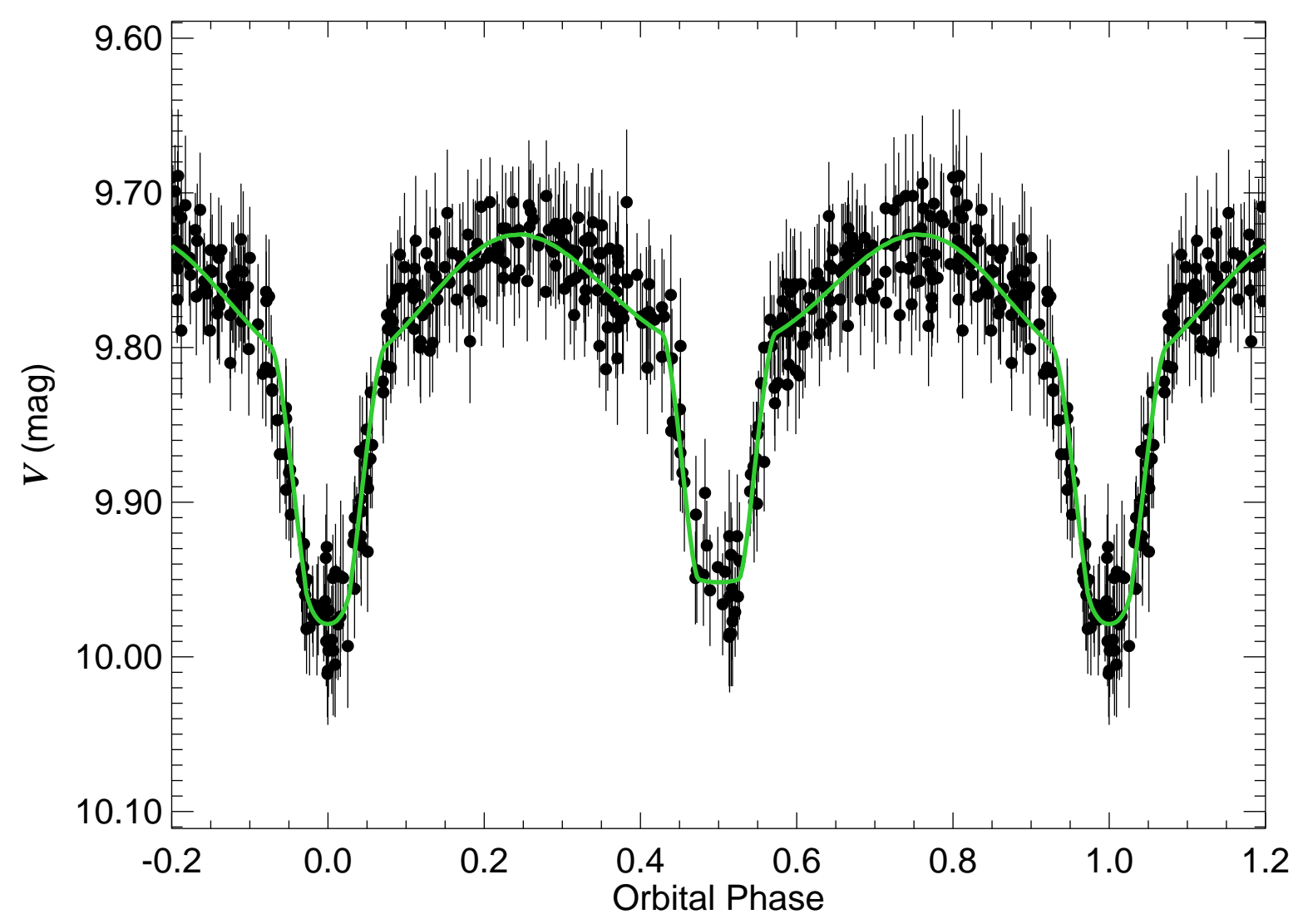

Fig. 55.- The ASAS $V$-band light curve for ASAS 183219-1117.4 (BD -11 4667). Filled circles with lines represent data with associated uncertainties. The best fit orbital solution listed in Table 2 is shown as a solid line passing through the data. 


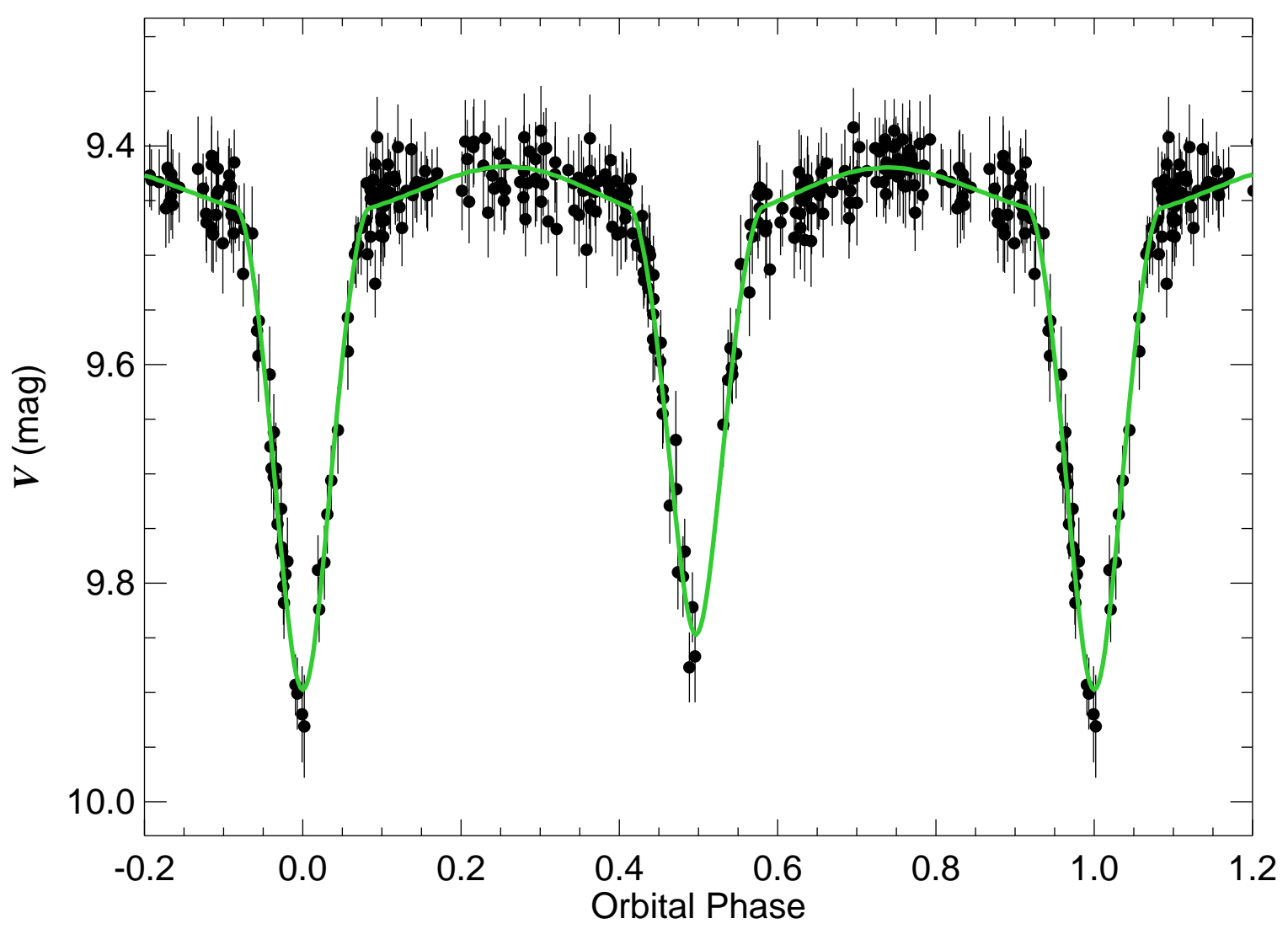

Fig. 56. - The ASAS $V$-band light curve for ASAS 184223+1158.9 (BD +11 3569). Filled circles with lines represent data with associated uncertainties. The best fit orbital solution listed in Table 2 is shown as a solid line passing through the data. 


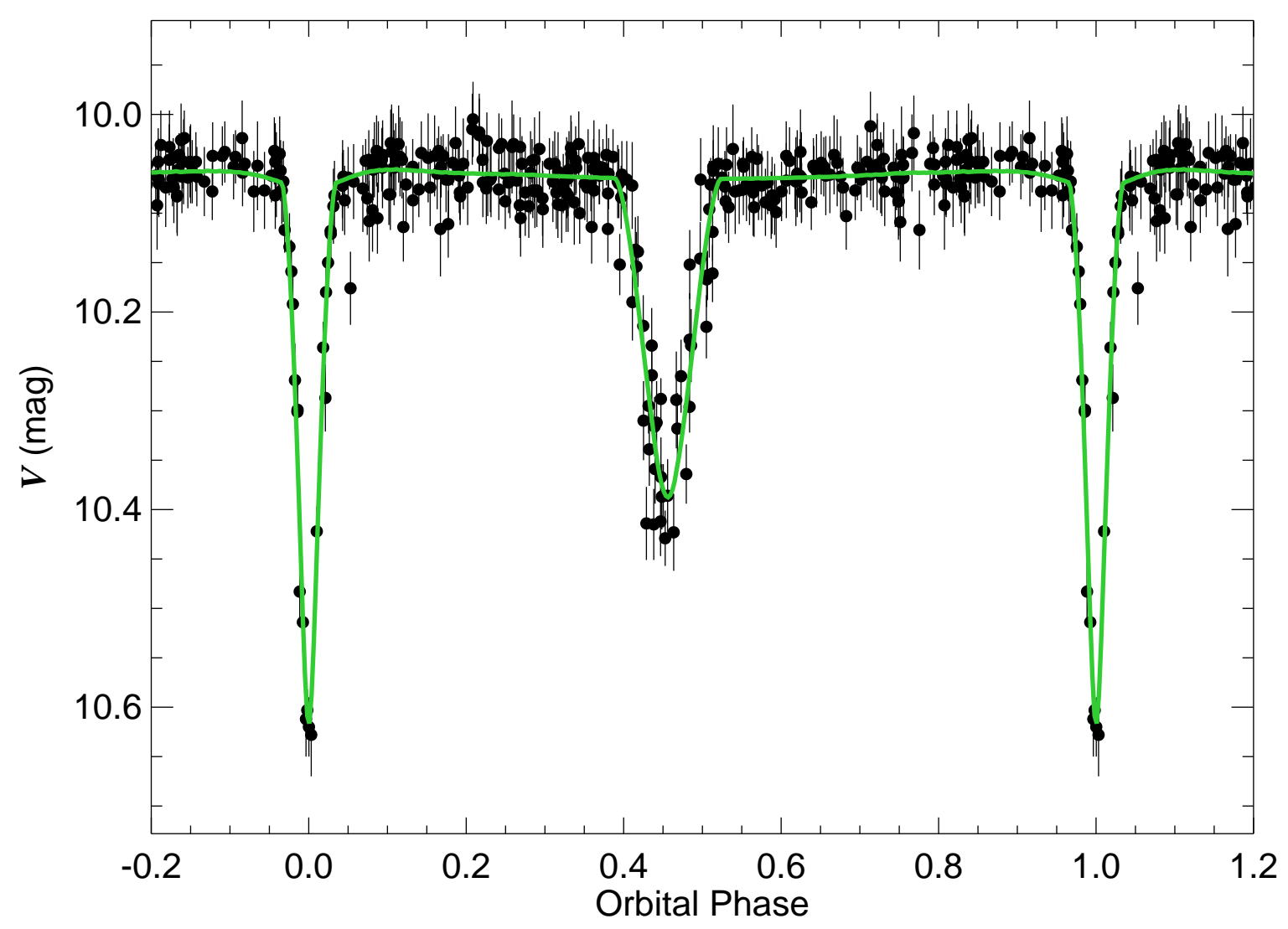

Fig. 57.- The ASAS $V$-band light curve for ASAS 184327+0841.5 (TYC 1025-1524-1). Filled circles with lines represent data with associated uncertainties. The best fit orbital solution listed in Table 2 is shown as a solid line passing through the data. 


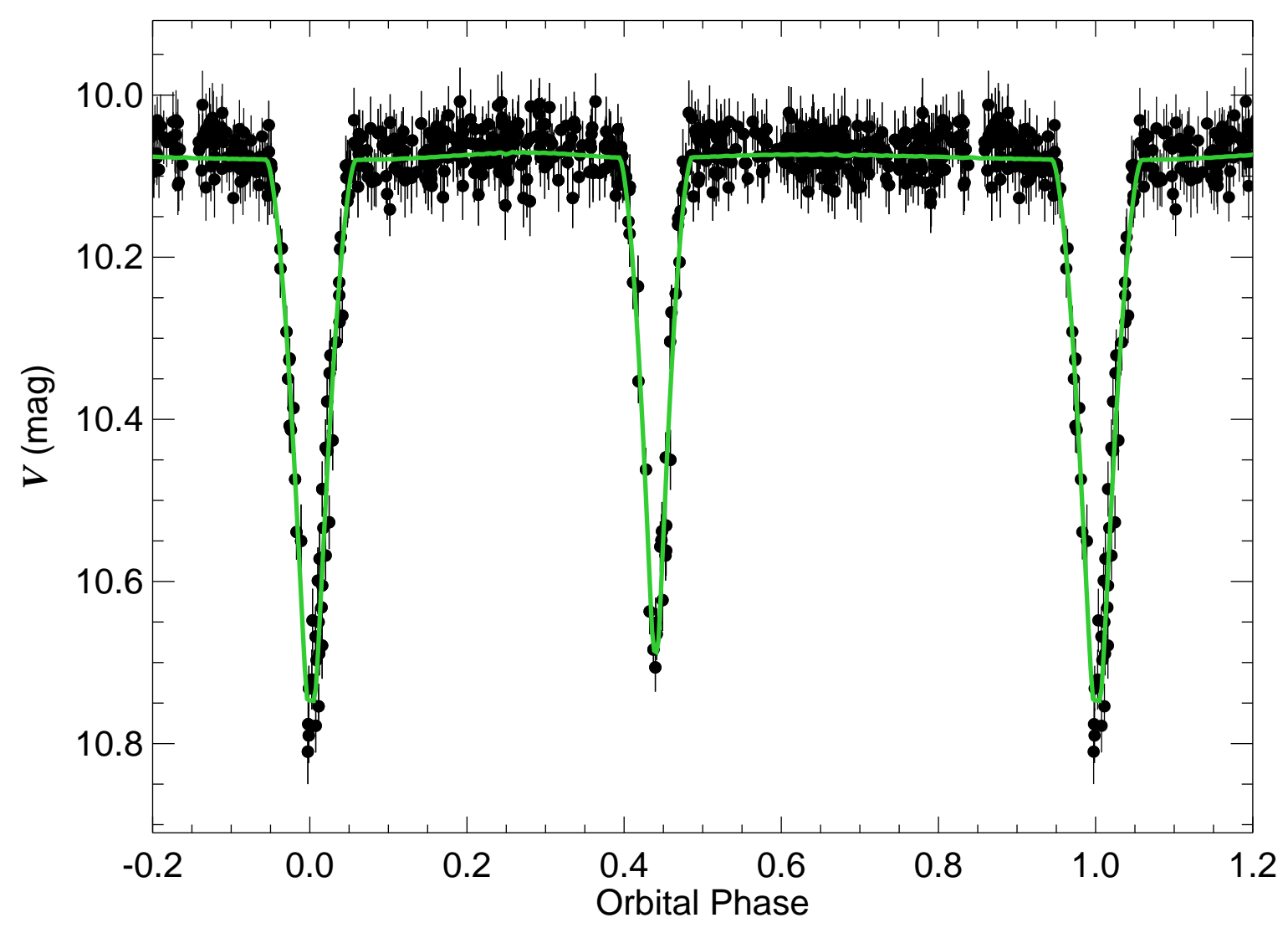

Fig. 58. - The ASAS $V$-band light curve for ASAS 184436-1923.4 (YY Sgr). Filled circles with lines represent data with associated uncertainties. The best fit orbital solution listed in Table 2 is shown as a solid line passing through the data. 


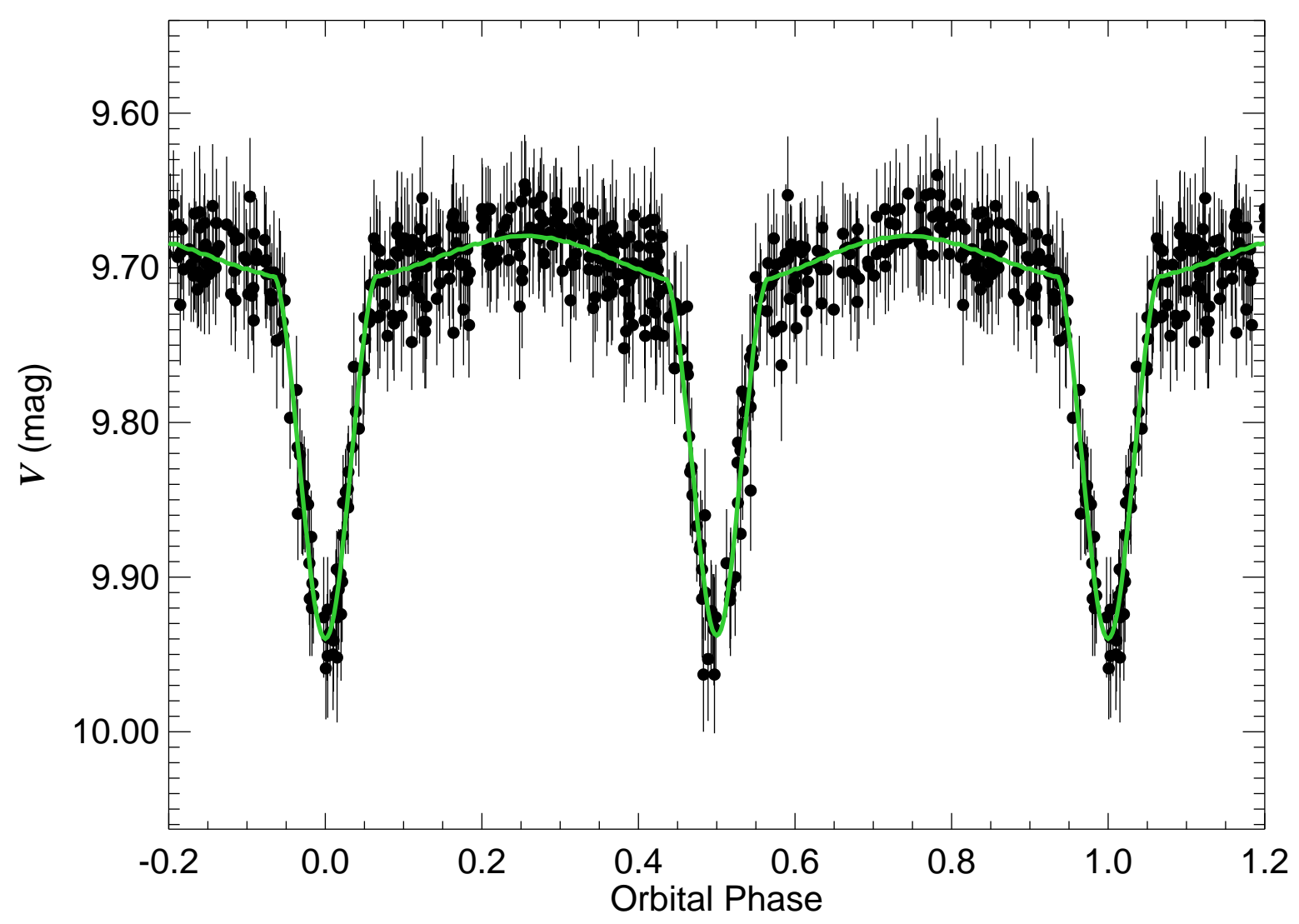

Fig. 59.- The ASAS $V$-band light curve for ASAS 185051-1354.6 (HD 174397). Filled circles with lines represent data with associated uncertainties. The best fit orbital solution listed in Table 2 is shown as a solid line passing through the data. 


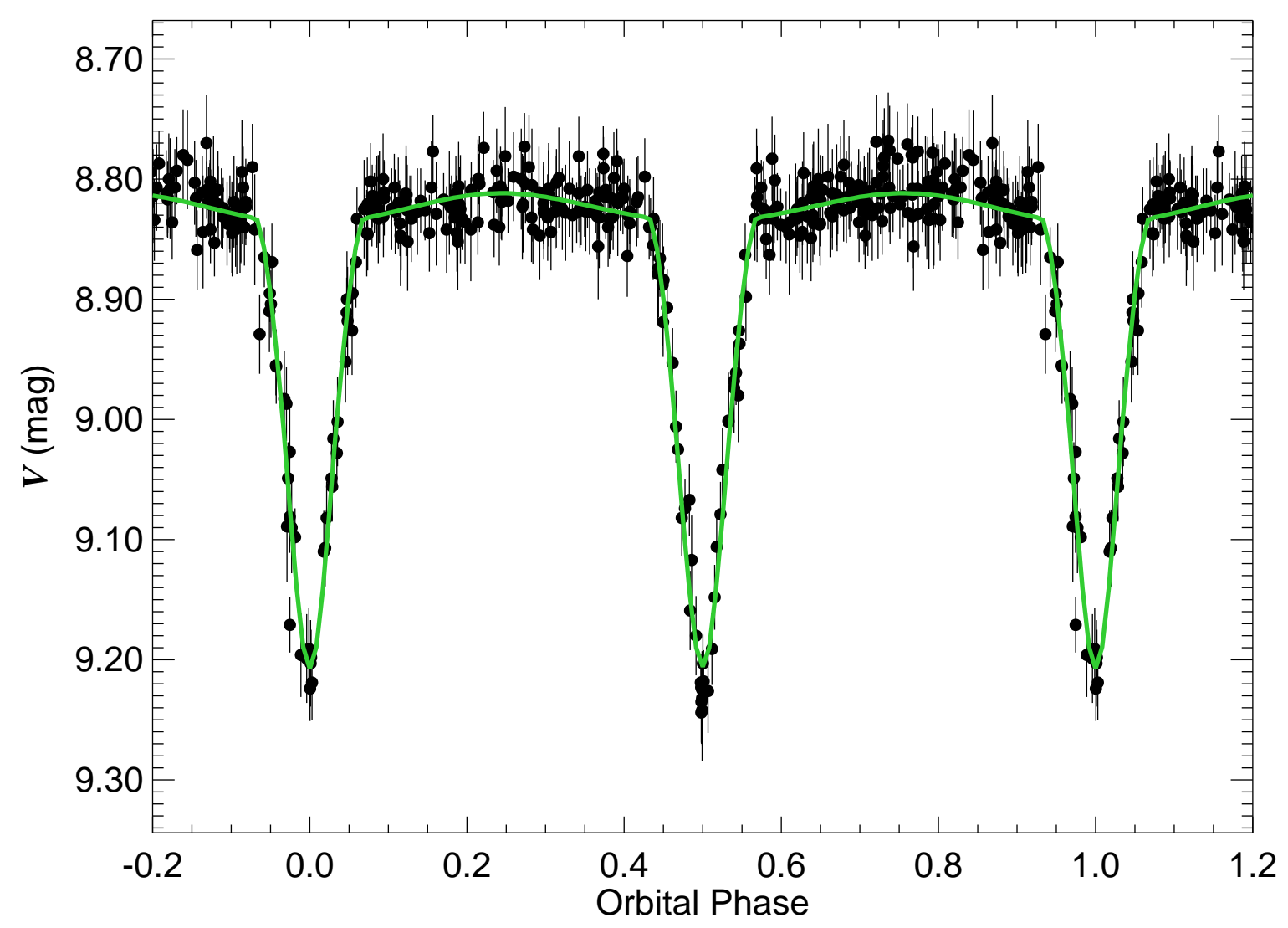

Fig. 60.- The ASAS $V$-band light curve for ASAS 194334-0904.0 (V1461 Aql). Filled circles with lines represent data with associated uncertainties. The best fit orbital solution listed in Table 2 is shown as a solid line passing through the data. 


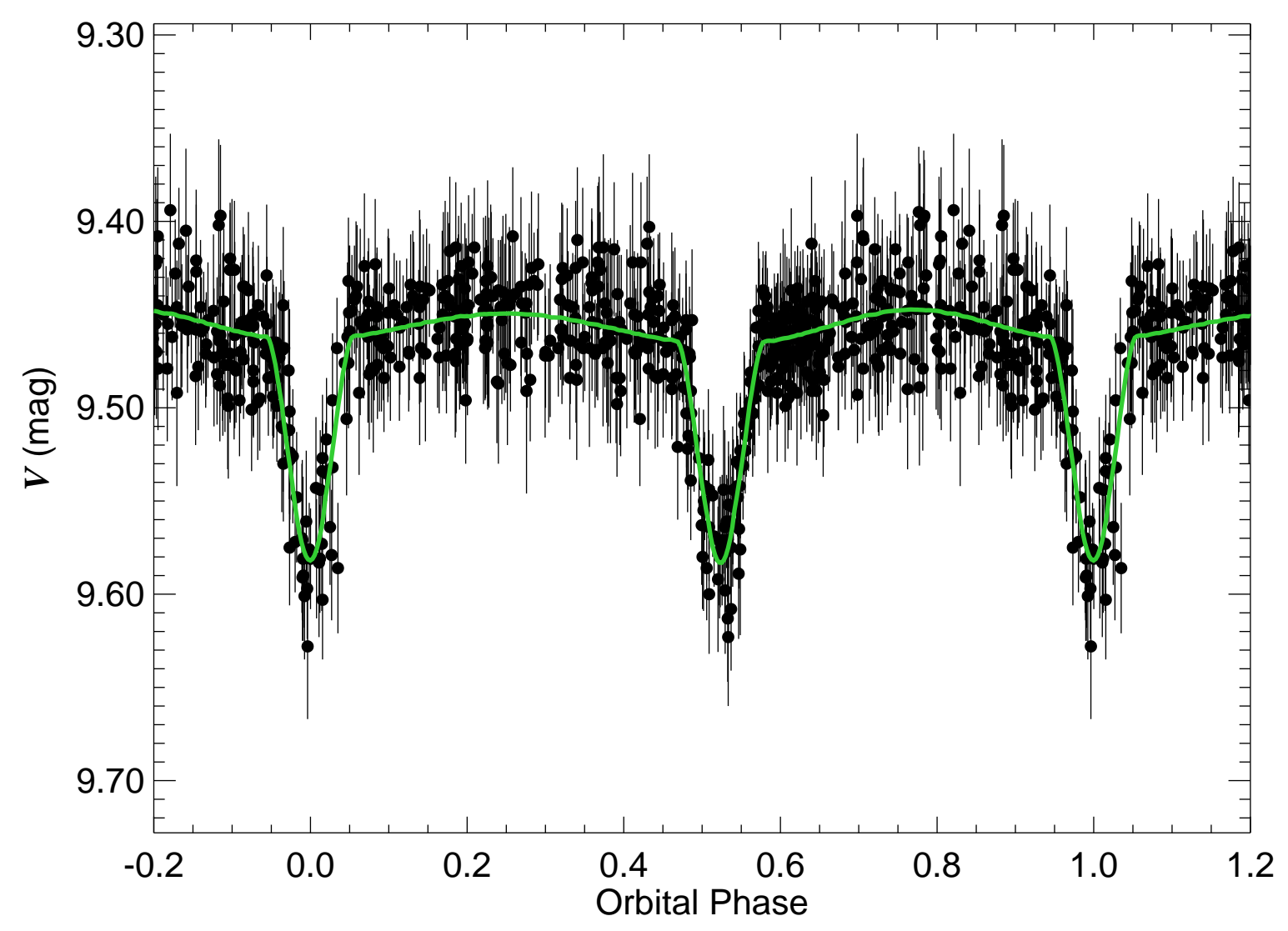

Fig. 61. - The ASAS $V$-band light curve for ASAS 195342+0205.4 (HD 188153). Filled circles with lines represent data with associated uncertainties. The best fit orbital solution listed in Table 2 is shown as a solid line passing through the data. 


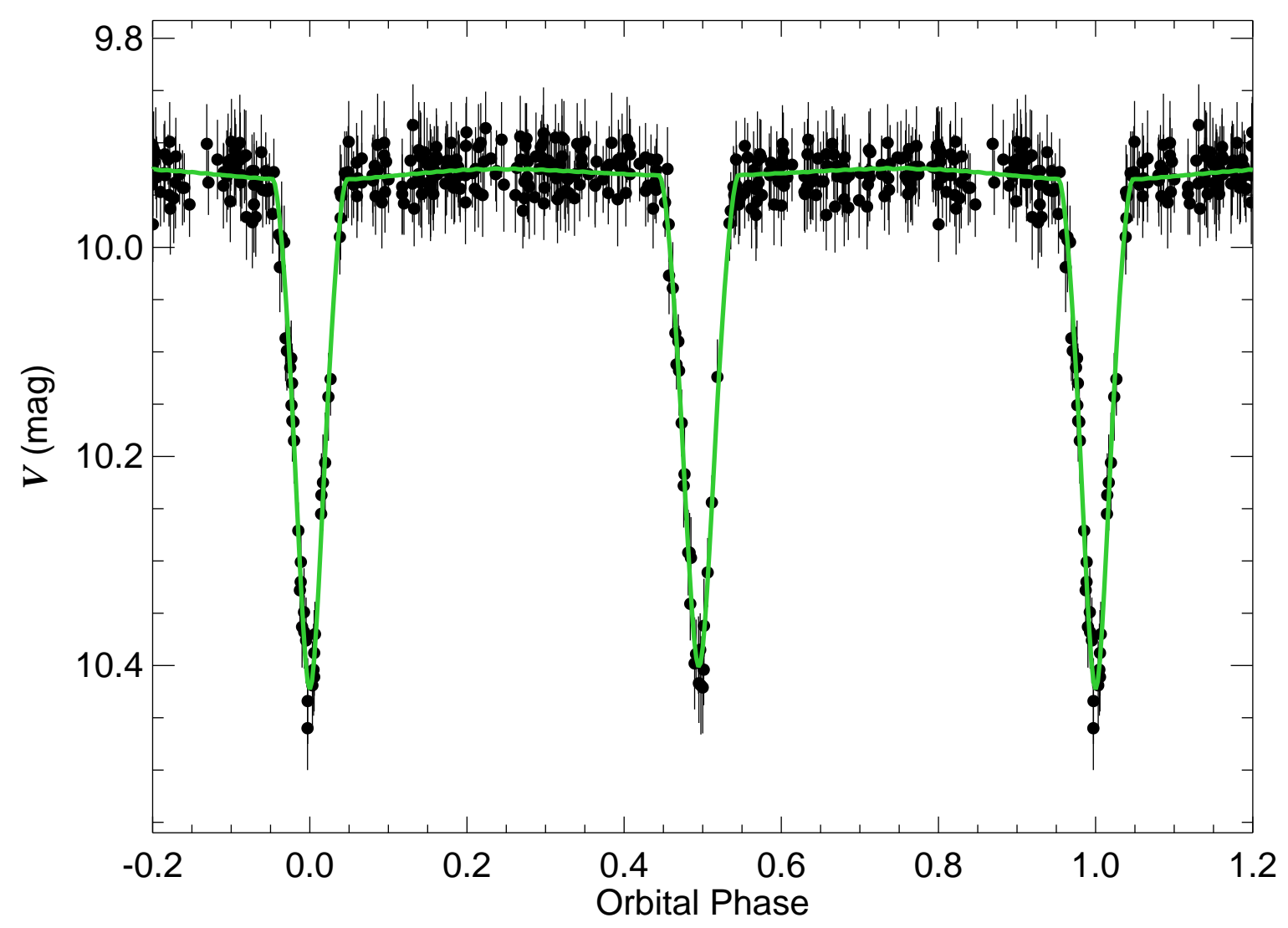

Fig. 62.- The ASAS $V$-band light curve for ASAS 195613+1630.9 (HD 354110). Filled circles with lines represent data with associated uncertainties. The best fit orbital solution listed in Table 2 is shown as a solid line passing through the data. 


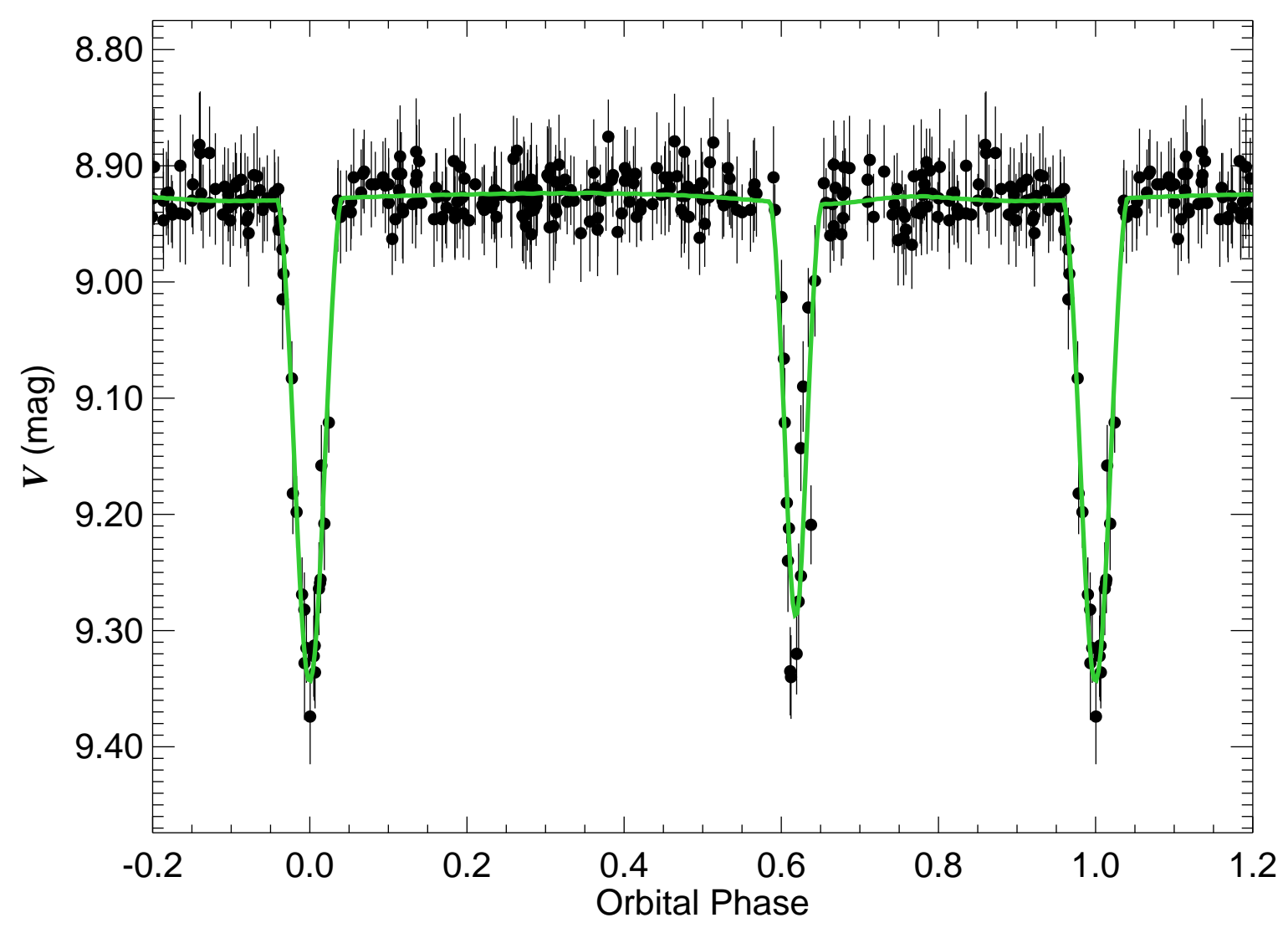

Fig. 63.- The ASAS $V$-band light curve for ASAS 205642+1153.0 (HD 199428). Filled circles with lines represent data with associated uncertainties. The best fit orbital solution listed in Table 2 is shown as a solid line passing through the data. 